\title{
Paleoproterozoic accretionary and collisional processes and the build-up of the Borborema Province (NE Brazil): Geochronological and geochemical evidence from the Central Domain
}

\author{
Sérgio P. Neves ${ }^{a,}{ }^{*}$, Geysson A. Lages ${ }^{b}$, Roberta G. Brasilino ${ }^{b}$, Alan W.A. Miranda ${ }^{c}$ \\ a Departamento de Geologia, Universidade Federal de Pernambuco, 50740-530 Recife, Brazil \\ ${ }^{\mathrm{b}}$ CPRM - Serviço Geológico Nacional, Av. Sul 2291, Afogados, Recife, PE 50770-0, Brazil \\ ${ }^{\mathrm{C}}$ Universidade Federal Rural do Rio de Janeiro, 21840-600 Rio de Janeiro, Brazil
}

\section{A R T I C L E I N F O}

Article history:

Received 7 March 2014

Accepted 27 June 2014

Available online 18 July 2014

\section{Keywords:}

$\mathrm{U}-\mathrm{Pb}$ geochronology

Geochemistry

Nd isotopes

Crustal evolution

\begin{abstract}
A B S T R A C T
Several Brasiliano-Pan-African belts consist of large areas of reworked Paleoproterozoic rocks. Characterization of these rocks is needed to place better controls on Precambrian paleogeographic reconstructions. The Borborema Province, northeastern Brazil, occupies a central position in West Gondwana configuration, and knowledge of its geological evolution is crucial to infer relationships between Paleoproterozoic units in South America and Africa. Here, we report $\mathrm{U}-\mathrm{Pb}$ ages, major- and trace-elements analyses and Sm-Nd isotopic data for orthogneisses in the eastern portion of Central Domain. The dominant basement units in the study area are banded gneisses of intermediate composition and relatively juvenile character, and migmatitic gneisses of granitic composition with Archean $\mathrm{Nd} \mathrm{T}_{\mathrm{DM}}$ model ages. One sample of the banded gneiss yielded a weighted ${ }^{207} \mathrm{~Pb} /{ }^{206} \mathrm{~Pb}$ age of $2096 \pm 23 \mathrm{Ma}$ and an upper intercept age of $2044 \pm 27 \mathrm{Ma}$, which we interpret, respectively, as ages of crystallization and metamorphism. Two large units of migmatitic gneiss in the southern and central parts of the area gave ages of, respectively, $2057 \pm 20 \mathrm{Ma}$ and $2055 \pm 23 \mathrm{Ma}$; an orthoamphibolite associated with the latter yielded crystallization age of $2042 \pm 11 \mathrm{Ma}$ and metamorphic age of $1996 \pm 13 \mathrm{Ma}$. All these rocks have geochemical signatures typical of subduction zone-related magmas. Combined with evidence provided by previous studies, we suggest that the evolution of the study area starts with island arc construction around $2.2 \mathrm{Ga}$, leading to an expressive volcanic arc edifice by 2.13 $-2.10 \mathrm{Ga}$. By $2.06 \mathrm{Ga}$, the crust had evolved enough to become intruded by magmas formed at the mantle wedge of the now largely continental magmatic arc, which continued to be intruded by mantle melts until at least $2.04 \mathrm{Ga}$. An augen gneiss in the northern part of the area, with an age of $2109 \pm 15 \mathrm{Ma}$, and a migmatitic gneiss with a much older age $(2183 \pm 9 \mathrm{Ma})$, both of which have geochemical characteristics akin to intraplate magmas, suggest that crustal reworking also took place. The $1981 \pm 23$ Ma age of a small body of peraluminous felsic gneiss may be related to regional metamorphism and syncollisional magmatism at c. 2.0-1.98 Ga.
\end{abstract}

(c) 2014 Elsevier Ltd. All rights reserved.

\section{Introduction}

The occurrence and extent of preexisting crust in orogenic belts has important implications for formulation of models of continental growth as well as for paleogeographic reconstructions. In the Gondwana realm, the presence of large fractions of reworked old continental crust in several Pan-African/Brasiliano belts was inferred long ago from reconnaissance geochronological data and/

\footnotetext{
* Corresponding author.

E-mail addresses: serpane36@yahoo.com, serpane@hotlink.com.br (S.P. Neves).
}

or structural/metamorphic characteristics (e.g., Bertrand and Laserre, 1976; Kroner, 1977; Almeida et al., 1981; Cordani et al., 1999). This assumption is becoming increasingly obvious as the number of precise geochronological data from specific areas accumulates (e.g., Bruguier et al., 1994; Ferré et al., 1996; Toteu et al., 2001; Njiosseu et al., 2005; Dada, 2008). Basement of Paleoproterozoic age in the Borborema Province, northeastern Brazil, was firmly established in the early 1990s (Hackspacher et al., 1990; Van Schmus et al., 1995). In the last decade, there was renewed emphasis on basic geological mapping, which coupled with $\mathrm{U}-\mathrm{Pb}$ zircon geochronology show that basement orthogneisses are mostly Paleoproterozoic in age (Neves et al., 2004, 2006; Souza 
(a)

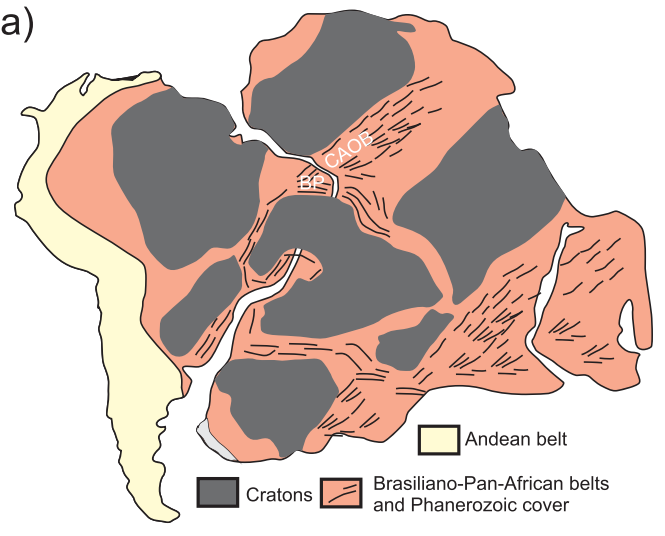

(b)

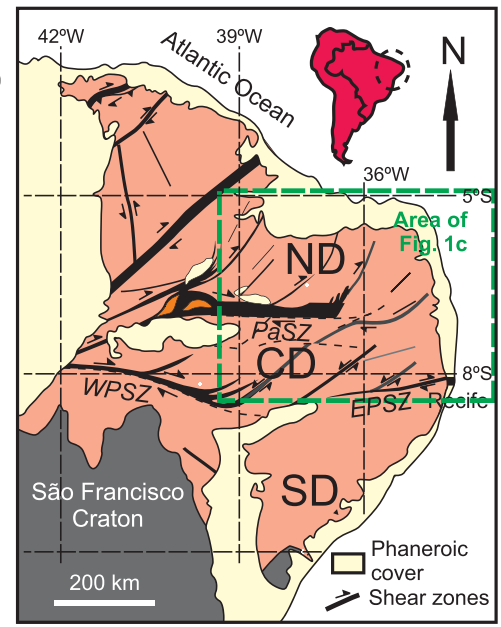

(c)

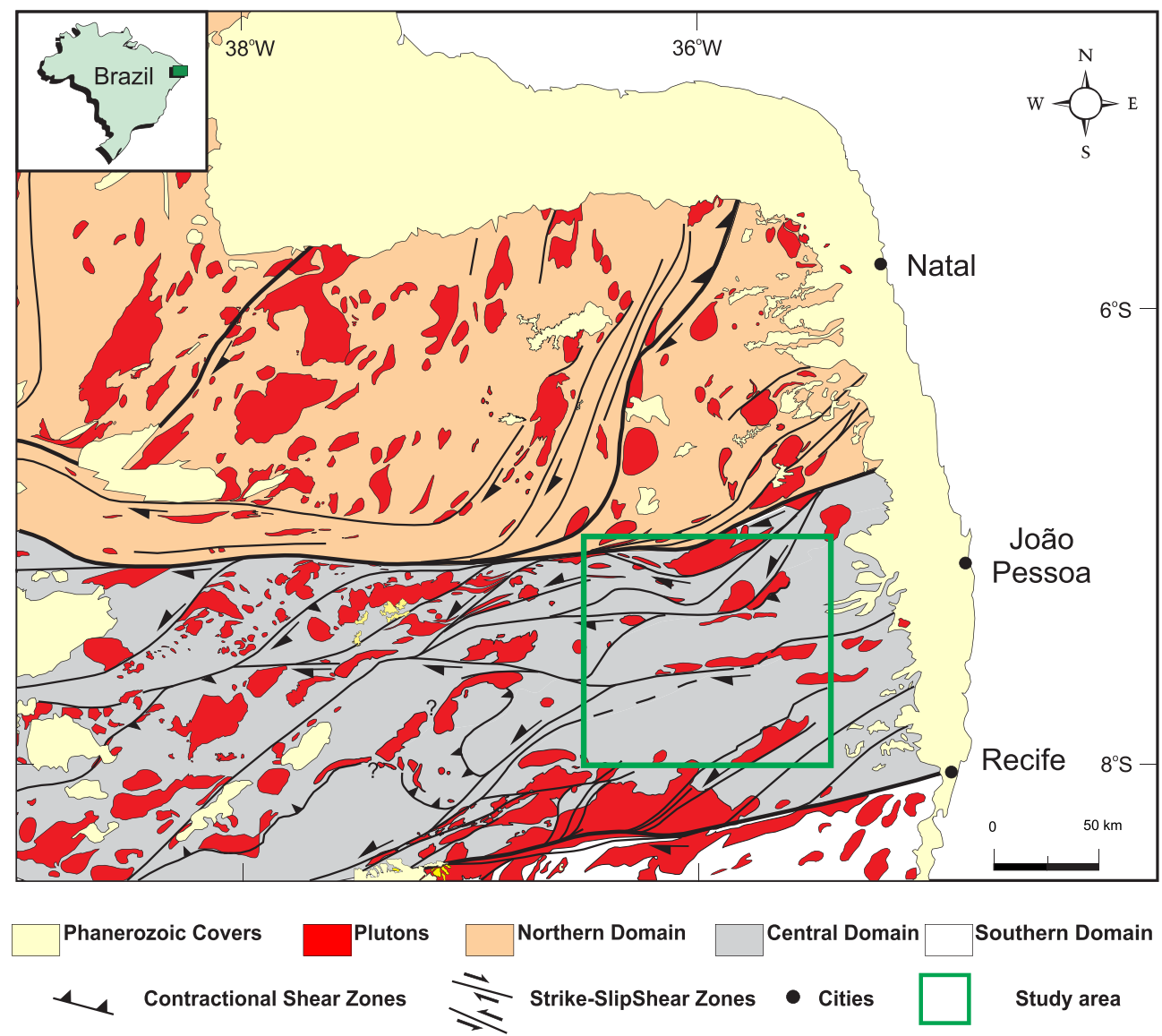

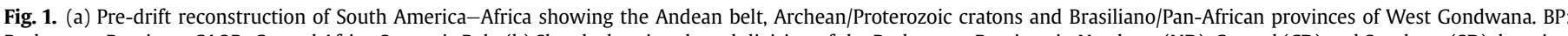

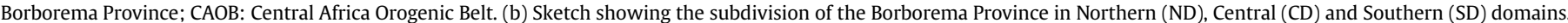

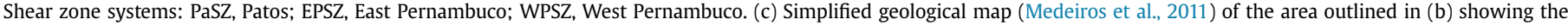
location of the study area.

et al., 2007; Arthaud et al., 2008; Santos et al., 2008; Van Schmus et al., 2008, 2011; Martins et al., 2009; Hollanda et al., 2011), although small Archean nuclei also exist (Dantas et al., 2004; Arthaud et al., 2008). Furthermore, U-Pb analysis of detrital zircons from metasedimentary sequences show Paleoproterozoic age peaks in several samples (Santos et al., 2004; Neves et al., 2006, 2009; Van Schmus et al., 2003, 2011; Araújo et al., 2012; Guimarães et al., 2012), indicating that Paleoproterozoic rocks were an important, if not dominant, source for the detritus. Finally, the presence of xenocristic zircons in Brasiliano granitoids and Sm-Nd model ages much older than their crystallization ages (Ferreira et al., 1998; Fetter et al., 2000; Mariano et al., 2001; Da Silva Filho et al., 2002; Hollanda et al., 2003; Guimarães et al. 2004; Rodrigues and Brito Neves, 2008; Van Schmus et al., 2008 2011; Neves et al., 2008) suggest that Paleoproterozoic rocks occur extensively in the deep crust. All these lines of evidence 

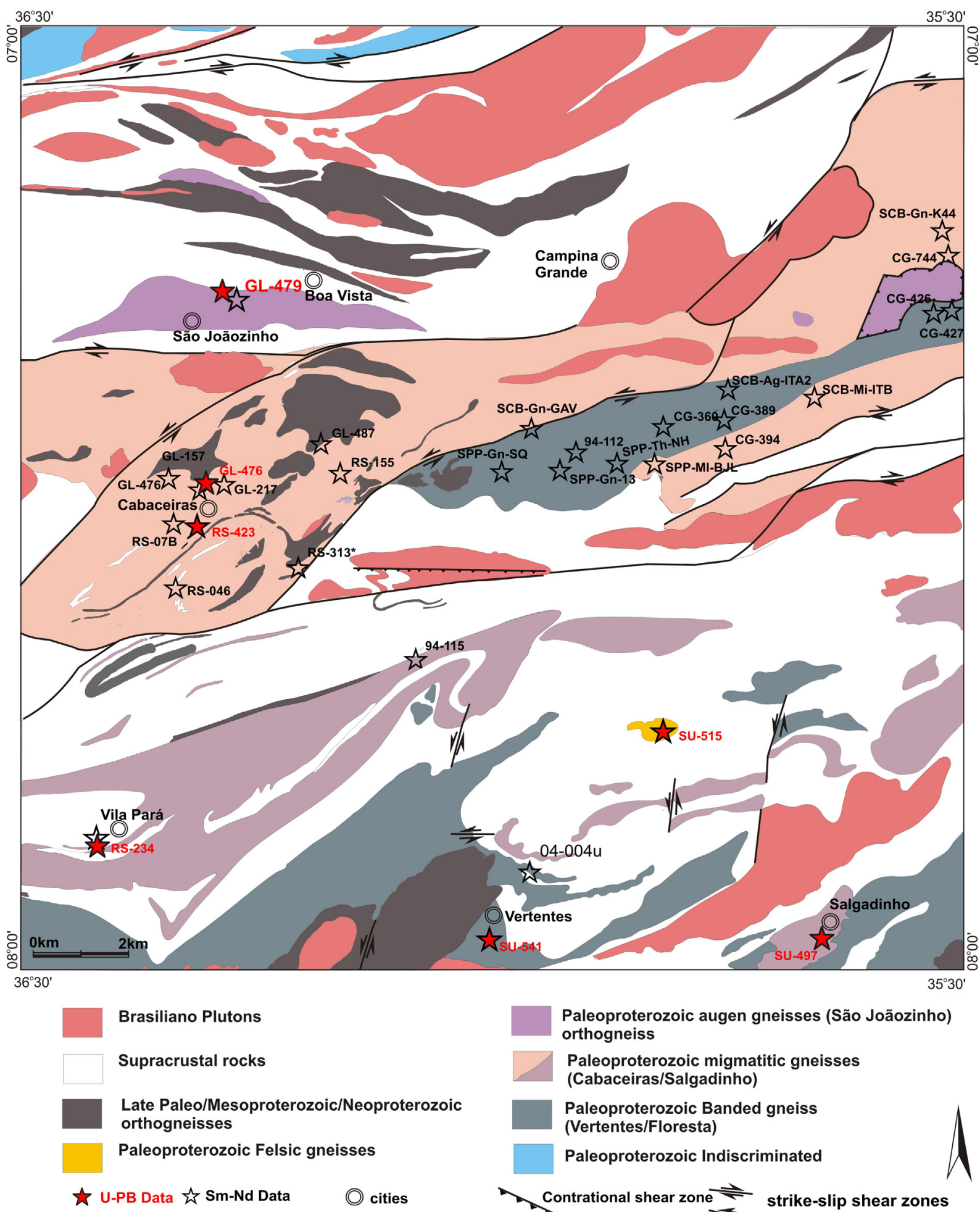

Paleoproterozoic augen gneisses (São Joãozinho) orthogneiss

Paleoproterozoic migmatitic gneisses (Cabaceiras/Salgadinho)

Paleoproterozoic Banded gneiss (Vertentes/Floresta)

Paleoproterozoic Indiscriminated

Contrational shear zone $\underset{F}{\longrightarrow}$ strike-slip shear zones

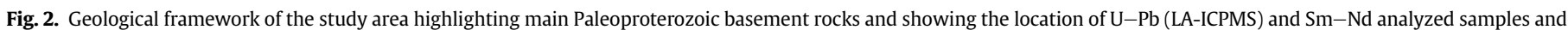
from available data. Compiled and modified from Neves et al. (2010), Rodrigues et al. (2010a), Brasilino et al. (2012), Lages and Marinho (2012).

confirm the Paleoproterozoic, particularly the $2.2-2.0$ b.y. time span, as the main period of crust formation and/or preservation in the Borborema Province.

The Borborema Province occupies a crucial position in West Gondwana, occurring between the Amazonian, São Luís and São Francisco-Congo cratons and westwards of the Central African
Orogenic Belt (Fig. 1a). Therefore, establishing correlations between basement rocks is needed in order to place better controls on paleogeographic reconstructions, not only for the Neoproterozoic but also for the Paleoproterozoic. In this contribution, we present new laser ablation multi-collector inductively coupled-mass spectrometry (LA-MC-ICP-MS) U-Pb zircon ages from orthogneisses in 
(a)

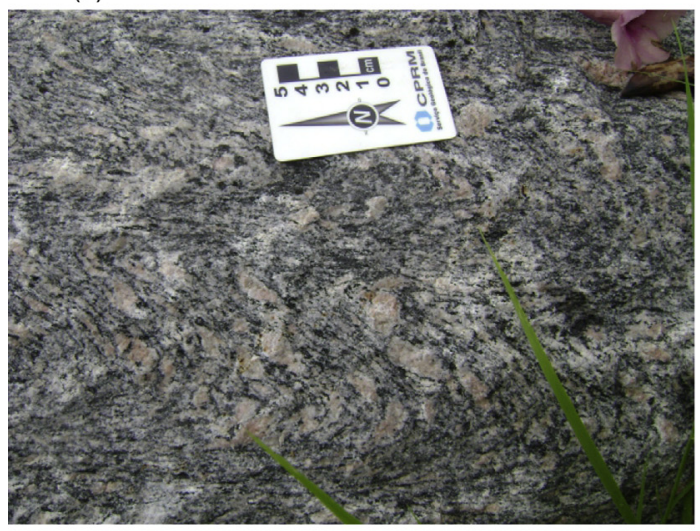

(c)

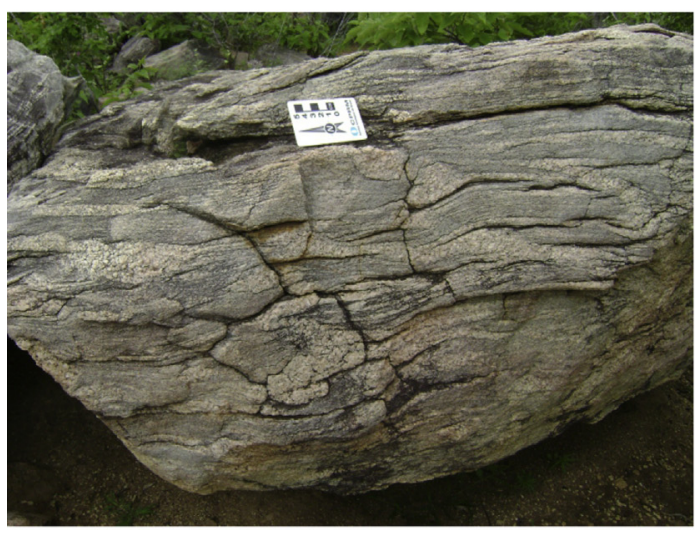

(e)

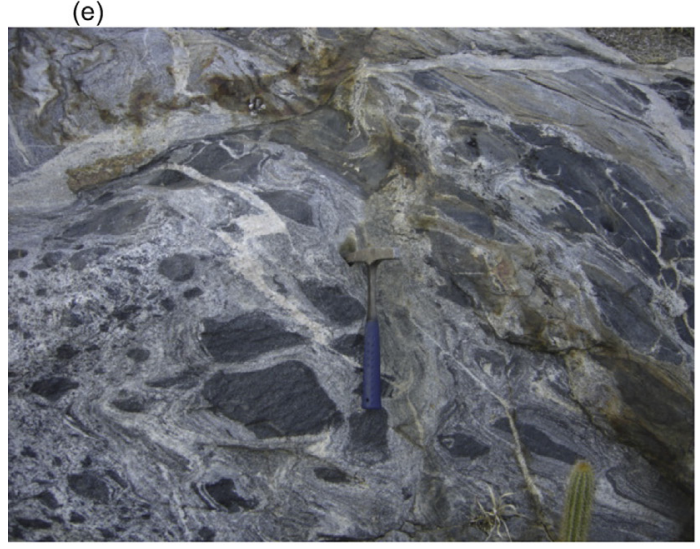

(g)

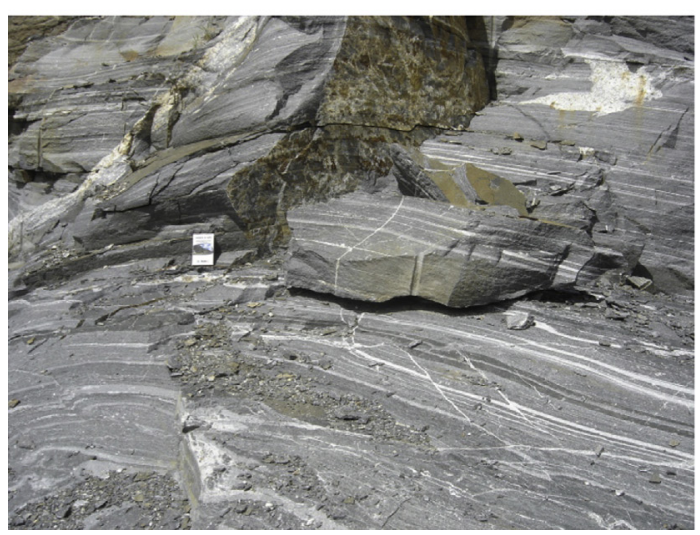

(b)

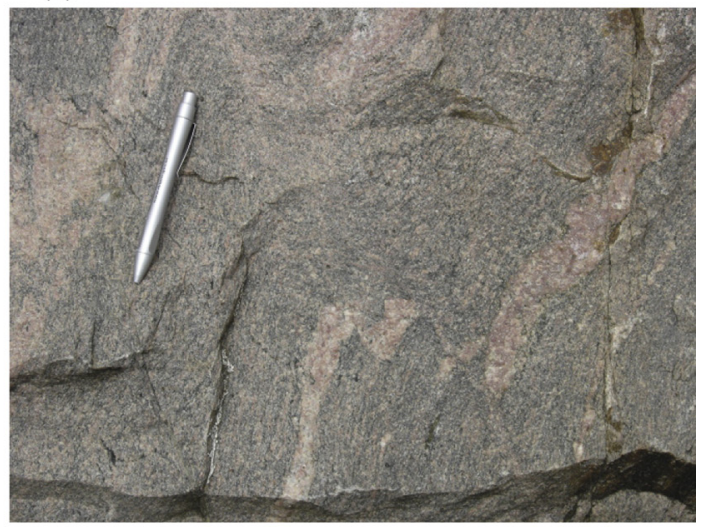

(d)
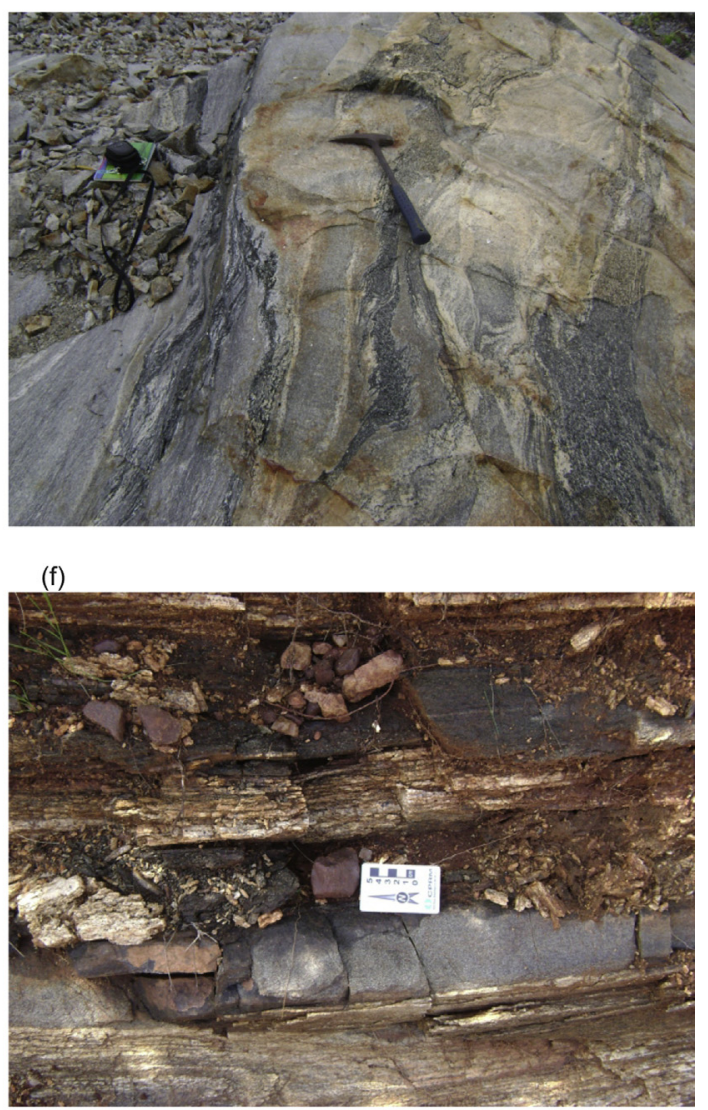

(h)

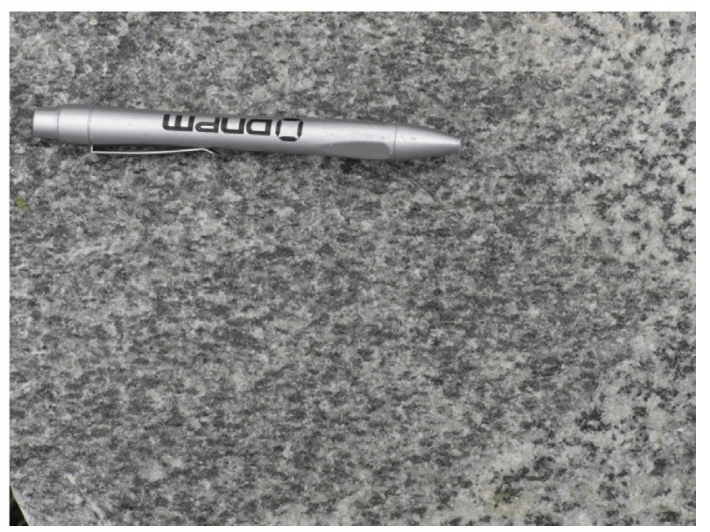


the eastern Borborema Province (Fig. 1b and c). Together with majorand trace elements and $\mathrm{Sm}-\mathrm{Nd}$ analyses, these data contribute to a better knowledge of the age and nature of Paleoproterozoic events that affected this area. We discuss the role of mantle and crustal sources for the magmatism, the possible environments of intrusion, the number and ages of metamorphic events, and, in conjunction with existing geochronological and geochemical data, propose a tectonic model for the evolution of the study area.

\section{Geological setting, petrography and previous $\mathrm{U}-\mathrm{Pb}$ results}

The study area is situated in the eastern portion of the Central Domain, whose limits are the large E-W-striking Patos and Pernambuco transcurrent shear zone systems (Fig. 1c). It comprises an area of about $12000 \mathrm{~km}^{2}$ that was mapped at the 1:100 000 scale (Fig. 2). In addition to the orthogneisses subject of this contribution (described below), the study area comprises late Paleoproterozoic to early Mesoproterozoic granitic orthogneisses, metagabbros and meta-anorthosites, small gneissic bodies of early Neoproterozoic age, amphibolite-facies metasedimentary rocks, rare granulitic rocks, and several granitic and syenitic plutons intruded during the Brasiliano orogeny. The orthogneissic basement was extensively reworked during this orogeny. A first deformation phase produced a shallowly to moderately dipping foliation shared by orthogneisses and metasedimentary rocks (Neves et al., 2000, 2005). A second main deformation event gave rise to conjugate sets of dextral, E-W-striking and sinistral, NE-SW-striking subvertical mylonitic belts (Neves and Vauchez, 1995; Neves and Mariano, 1999; Archanjo et al., 2008).

According to their field and petrographic characteristics, the Paleoproterozoic orthogneisses in the study area can be classified into four main groups. The first comprises non-migmatitic and dominantly augen gneisses (Fig. 3a), locally known as São Joãozinho orthogneiss, and is located in the northwestern portion of the area. . It is a biotite-amphibole-bearing gneiss with syenogranitic to monzogranitic composition. The main foliation is defined by stretched quartz that wraps around K-feldspar porphyroclasts. Metamorphism under amphibolite facies conditions is suggested by the association recrystallized K-feldspar + biotite + plagioclase + quartz + titanite. Rodrigues et al. (2010) obtained a U-Pb concordia age of $2137 \pm 21 \mathrm{Ma}$ for one sample of augen gneiss whereas two samples dated by Santos et al. (2013) yielded complex results, with ${ }^{207} \mathrm{~Pb} /{ }^{206} \mathrm{~Pb}$ ages varying from 2.3 to $1.95 \mathrm{Ga}$.

The second group of orthogneisses includes migmatized amphibole-biotite-bearing granitic gneisses (Fig. 3b, c, d). They are dominantly coarse-grained (although augen gneisses also occur) and characterized in several outcrops by magnetite-bearing pinkish leucosomes that may be either parallel to or truncate the main foliation. Centimetric to decimetric amphibolitic layers or rounded to elliptic metagabbroic/amphibolitic enclaves occur frequently associated with this group (Fig. 3e). Metagabbroanorthosites, metadiorites, orthoamphibolites (Fig.3f) and massive titanium magnetite ore bodies can also occur locally. In the augen gneisses the fabric is sometimes mylonitic and consists of stretched quartz wrapping around feldspar porphyroclasts. The main mafic phase is amphibole with optical characteristics of hornblende. The paragenesis amphibole + biotite \pm (rare)pyroxene + recrystallized plagioclase and $\mathrm{K}$-feldspar + quartz + titanite + opaque minerals reflects amphibolite facies conditions. In some samples, a retrometamorphic assemblage is evidenced by destabilization of pyroxene to amphibole with bluish-green pleochroism that is in turn replaced by epidote, and by replacement of plagioclase by epidote, quartz and calcite. Reaction of hornblende + biotite (first generation) \pm plagioclase giving rise to garnet + biotite (second generation) \pm microcline suggests maintenance of high temperature conditions during at least two metamorphic events. Assessment of geochemical data (Section 4) demonstrates that these migmatitic orthogneisses belong to three distinct units. We use the terms Salgadinho and Cabaceiras for orthogneissic complexes occurring, respectively, in the southern and central parts of the study area. Santos et al. (2004) obtained an upper intercept age at $2016 \pm 27 \mathrm{Ma}(\mathrm{MSWD}=0.6)$ in a biotite-amphibole bearing granitic orthogneiss that we correlated with this group.

The third group of orthogneisses comprises banded gneisses. They dominate in the southern portion of the study area and are referred to as Vertentes Complex around the homologous city and as Floresta Complex elsewhere. These orthogneisses are characterized by regular, centimetric to decimetric, alternating bands with dioritic (dominant) to granitic compositions (Fig. 3g). Finegrained mafic layers and migmatized portions with concordant and discordant leucosomes may occur locally. Intercalated metamafic rocks (amphibolites and garnet amphibolites), varying from centimetric/decimetric bands to kilometer-long lenses, are a major characteristic of this unit. In the mafic/intermediate bands, the mineralogical assemblage consists of plagioclase, quartz, K-feldspar, biotite, amphibole, garnet and, occasionally, clinopyroxene. Apatite, titanite, allanite and zircon are accessory minerals. Hypidiomorphic prismatic plagioclase crystals with parallel twins and inclusions indicate igneous protolith. Porphyroblastic garnet tends to engulf plagioclase and amphibole from the matrix, indicating metamorphic origin, and may show retrogression to opaque minerals + chlorite. One sample dated by Sá et al. (2002) provided a discordia with upper intercept age of $1970 \pm 30$ Ma but with a very large MSWD (30). Neves et al. (2006) dated the mafic and felsic portions of a banded sample. In the mafic band they obtained two groups of ages, averaging $2127 \pm 7 \mathrm{Ma}$ and $2044 \pm 5 \mathrm{Ma}$. These results were interpreted as ages of crystallization and metamorphism, respectively. One zircon from the felsic band yielded an age of $625 \pm 24 \mathrm{Ma}$, which was inferred as crystallization age and, therefore, the acquisition of the banded structure during the Brasiliano orogeny. A sample of banded gneiss dated by Brito Neves et al. (2013) gave a crystallization age of $2162 \pm 7$ Ma. In another sample, a few kilometers to the east of the study area, Brito Neves et al. (2013) report crystallization age of $2110 \pm 7$ Ma, with metamorphic overgrowth at $1983 \pm 20 \mathrm{Ma}$. Analyses of zircons from two samples of granodioritic composition, relatively more homogeneous, defined discordia lines with upper intercepts of $2103 \pm 11 \mathrm{Ma}$ (Neves et al., 2006) and $2096 \pm 7 \mathrm{Ma}$ (Brito Neves et al., 2013). Lower intercepts of $619 \pm 36 \mathrm{Ma}$ and $588 \pm 53 \mathrm{Ma}$, respectively, in these samples confirm Neoproterozoic superimposed metamorphism.

The fourth group of orthogneisses is subordinate in abundance and comprises equigranular felsic granitic gneisses (Fig. $3 \mathrm{~h}$ ) that, in contrast with the previous groups, do not contain amphibole. Sixteen near concordant analyses of zircon grains from one sample dated by Neves et al. (2006) yielded a well-constrained ${ }^{207} \mathrm{~Pb} /{ }^{206} \mathrm{~Pb}$

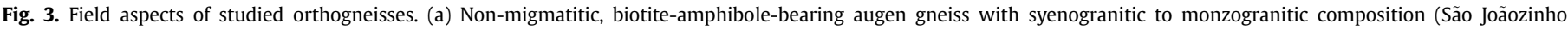

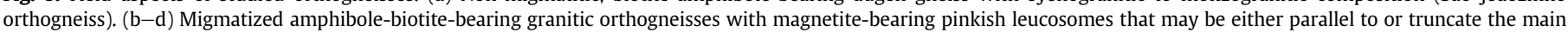

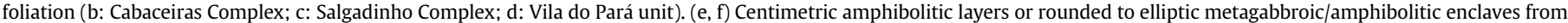

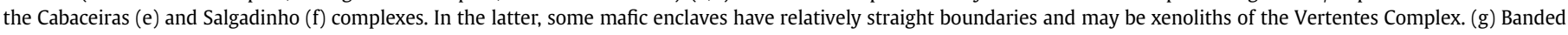

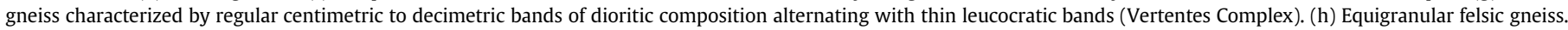


Table 1

LA-ICPMS U-Th-Pb results for zircons from samples of plutonic rocks of the basement of eastern Borborema Province.

\begin{tabular}{|c|c|c|c|c|c|c|c|c|c|c|c|c|c|c|c|c|}
\hline \multirow[b]{2}{*}{ Samples } & \multirow[t]{3}{*}{ Th/U } & \multicolumn{7}{|l|}{ Ratios } & \multirow[t]{3}{*}{$\rho$} & Apparen & ages & & & & & Conc. (\%) \\
\hline & & $206 \mathrm{~Pb}$ & $207 \mathrm{~Pb}$ & $\pm 1 \sigma$ & $207 \mathrm{~Pb}$ & $\pm 1 \sigma$ & $206 \mathrm{~Pb}$ & $\pm 1 \sigma$ & & $207 \mathrm{~Pb}$ & (Ma) & $207 \mathrm{~Pb}$ & (Ma) & $206 \mathrm{~Pb}$ & (Ma) & \\
\hline Spots & & $204 \mathrm{~Pb}$ & $206 \mathrm{~Pb}$ & & $235 U$ & & $238 U$ & & & $206 \mathrm{~Pb}$ & & $235 \mathrm{U}$ & & $238 \mathrm{U}$ & & \\
\hline SU-497 (S & lgadin & & & & & & & & & & & & & & & \\
\hline 003-Z01 & 0.07 & 178148 & 0.11595 & 0.45 & 3.2984 & 1.68 & 0.20632 & 1.61 & 0.96 & 1895 & 8 & 1481 & 13 & 1209 & 17.78 & 81.66 \\
\hline 004-Z02 & 0.20 & 87087 & 0.11438 & 0.50 & 4.1292 & 1.93 & 0.26183 & 1.86 & 0.96 & 1870 & 9 & 1660 & 16 & 1499 & 24.85 & 90.31 \\
\hline 016-Z11 & 0.35 & 105234 & 0.13075 & 0.67 & 6.0957 & 1.61 & 0.33812 & 1.46 & 0.91 & 2108 & 12 & 1990 & 14 & 1878 & 23.81 & 94.37 \\
\hline 024-Z16 & 0.31 & 205444 & 0.12680 & 0.61 & 6.3123 & 1.37 & 0.36106 & 1.22 & 0.93 & 2054 & 11 & 2020 & 12 & 1987 & 20.86 & 98.37 \\
\hline 021-Z13 & 0.24 & 467687 & 0.12511 & 0.67 & 6.5208 & 1.73 & 0.37802 & 1.60 & 0.92 & 2030 & 12 & 2049 & 15 & 2067 & 28.28 & 100.89 \\
\hline 006-Z04 & 0.36 & 413082 & 0.12754 & 0.60 & 6.6486 & 1.09 & 0.37808 & 0.91 & 0.89 & 2064 & 11 & 2066 & 10 & 2067 & 16.08 & 100.07 \\
\hline 005-Z03 & 0.34 & 397166 & 0.12790 & 0.44 & 6.6630 & 1.34 & 0.37784 & 1.27 & 0.94 & 2069 & 8 & 2068 & 12 & 2066 & 22.39 & 99.92 \\
\hline 010-Z06 & 0.33 & 905425 & 0.12616 & 0.49 & 6.8494 & 1.08 & 0.39375 & 0.97 & 0.88 & 2045 & 9 & 2092 & 10 & 2140 & 17.60 & 102.30 \\
\hline 027-Z17 & 0.23 & 328175 & 0.12859 & 0.56 & 6.8736 & 1.22 & 0.38767 & 1.09 & 0.88 & 2079 & 10 & 2095 & 11 & 2112 & 19.54 & 100.80 \\
\hline 017-Z12 & 0.26 & 132399 & 0.12911 & 0.78 & 6.9536 & 1.18 & 0.39062 & 0.88 & 0.73 & 2086 & 14 & 2106 & 10 & 2126 & 16.02 & 100.96 \\
\hline 023-Z15 & 0.17 & 598069 & 0.12797 & 0.44 & 7.0641 & 1.05 & 0.40037 & 0.95 & 0.90 & 2070 & 8 & 2120 & 9 & 2171 & 17.51 & 102.42 \\
\hline 011-Z07 & 0.20 & 126701 & 0.12744 & 0.41 & 7.0785 & 1.19 & 0.40286 & 1.12 & 0.94 & 2063 & 7 & 2121 & 11 & 2182 & 20.68 & 102.87 \\
\hline 015-Z09 & 0.10 & 1038519 & 0.12902 & 0.66 & 7.1481 & 1.29 & 0.40183 & 1.11 & 0.85 & 2085 & 12 & 2130 & 11 & 2177 & 20.45 & 102.23 \\
\hline 009-Z05 & 0.37 & 546956 & 0.12667 & 2.47 & 7.1968 & 2.60 & 0.41205 & 0.82 & 0.29 & 2052 & 44 & 2136 & 23 & 2224 & 15.37 & 104.13 \\
\hline 012-Z08 & 0.27 & 855232 & 0.12653 & 1.01 & 7.3244 & 1.21 & 0.41985 & 0.67 & 0.70 & 2050 & 18 & 2152 & 11 & 2260 & 12.72 & 105.02 \\
\hline 030-Z20 & 0.56 & 2183059 & 0.12920 & 1.87 & 7.4432 & 2.47 & 0.41784 & 1.62 & 0.85 & 2087 & 33 & 2166 & 22 & 2251 & 30.68 & 103.90 \\
\hline 029-Z19 & 0.58 & 1055276 & 0.12832 & 0.98 & 7.7982 & 1.70 & 0.44076 & 1.39 & 0.81 & 2075 & 17 & 2208 & 15 & 2354 & 27.40 & 106.61 \\
\hline 022-Z14 & 0.33 & 419052 & 0.13096 & 0.49 & 7.8153 & 1.09 & 0.43280 & 0.97 & 0.88 & 2111 & 9 & 2210 & 10 & 2318 & 18.92 & 104.90 \\
\hline 028-Z18 & 0.33 & 526867 & 0.12806 & 0.60 & 7.8227 & 1.99 & 0.44303 & 1.89 & 0.95 & 2072 & 11 & 2211 & 18 & 2364 & 37.44 & 106.94 \\
\hline GL-476 (C & baceir & & & & & & & & & & & & & & & \\
\hline z01 & 0.13 & 95073 & 0.121497 & 1.71 & 5.022 & 1.31 & 0.299814 & 1.10 & 0.68 & 1978 & 30 & 1823 & 11 & 1690 & 16.41 & 85.45 \\
\hline z02 & 0.18 & 78340 & 0.121050 & 0.64 & 5.047 & 1.28 & 0.302399 & 1.10 & 0.71 & 1972 & 11 & 1827 & 11 & 1703 & 16.53 & 86.38 \\
\hline Z03 & 0.14 & 48325 & 0.100339 & 3.49 & 2.513 & 2.59 & 0.181664 & 2.34 & 0.87 & 1630 & 63 & 1276 & 19 & 1076 & 23.16 & 66.00 \\
\hline z04 & 0.19 & 60637 & 0.119107 & 1.81 & 4.560 & 1.33 & 0.277694 & 1.23 & 0.82 & 1943 & 32 & 1742 & 11 & 1580 & 17.17 & 81.31 \\
\hline z05 & 0.22 & 121456 & 0.124343 & 0.47 & 6.338 & 1.13 & 0.369662 & 1.03 & 0.81 & 2019 & 8 & 2024 & 10 & 2028 & 17.87 & 100.41 \\
\hline z06 & 0.19 & 15830 & 0.126051 & 1.59 & 5.788 & 1.21 & 0.333015 & 1.03 & 0.71 & 2044 & 28 & 1945 & 10 & 1853 & 16.56 & 90.67 \\
\hline Z07 & 0.13 & 122288 & 0.119169 & 2.97 & 5.023 & 2.15 & 0.305720 & 2.06 & 0.92 & 1944 & 52 & 1823 & 18 & 1720 & 30.95 & 88.47 \\
\hline z08 & 0.13 & 115157 & 0.124472 & 0.59 & 6.020 & 1.13 & 0.350781 & 0.97 & 0.69 & 2021 & 10 & 1979 & 10 & 1938 & 16.17 & 95.89 \\
\hline z09core & 0.09 & 252976 & 0.128337 & 0.81 & 6.519 & 1.39 & 0.368410 & 1.13 & 0.61 & 2075 & 14 & 2048 & 12 & 2022 & 19.67 & 97.43 \\
\hline z10 & 0.18 & 47411 & 0.123179 & 0.58 & 5.334 & 0.98 & 0.314075 & 0.79 & 0.62 & 2003 & 10 & 1874 & 8 & 1761 & 12.19 & 87.91 \\
\hline z11 & 0.13 & 104272 & 0.123565 & 1.69 & 5.740 & 1.25 & 0.336928 & 1.13 & 0.77 & 2008 & 30 & 1937 & 11 & 1872 & 18.32 & 93.21 \\
\hline z13 & 0.11 & 58659 & 0.117420 & 1.87 & 4.596 & 1.38 & 0.283860 & 1.27 & 0.80 & 1917 & 33 & 1748 & 11 & 1611 & 18.05 & 84.01 \\
\hline z14 & 0.14 & 75793 & 0.117193 & 2.50 & 4.209 & 1.87 & 0.260466 & 1.66 & 0.84 & 1914 & 44 & 1676 & 15 & 1492 & 22.02 & 77.97 \\
\hline z15 & 0.15 & 114088 & 0.123412 & 1.44 & 5.740 & 1.10 & 0.337329 & 0.92 & 0.75 & 2006 & 25 & 1937 & 9 & 1874 & 15.01 & 93.40 \\
\hline z16 & 0.13 & 191156 & 0.123172 & 0.53 & 5.622 & 1.04 & 0.331037 & 0.89 & 0.76 & 2003 & 9 & 1919 & 9 & 1843 & 14.34 & 92.05 \\
\hline z17 & 0.25 & 63603 & 0.119684 & 2.12 & 4.976 & 1.56 & 0.301532 & 1.44 & 0.81 & 1952 & 37 & 1815 & 13 & 1699 & 21.47 & 87.06 \\
\hline z18 & 0.10 & 70653 & 0.119237 & 2.60 & 4.847 & 1.91 & 0.294792 & 1.76 & 0.88 & 1945 & 46 & 1793 & 16 & 1665 & 25.84 & 85.63 \\
\hline z19 & 0.32 & 58475 & 0.124626 & 2.01 & 5.031 & 1.51 & 0.292773 & 1.33 & 0.81 & 2023 & 35 & 1825 & 13 & 1655 & 19.41 & 81.81 \\
\hline$z 20$ & 0.17 & 64607 & 0.116724 & 0.61 & 3.987 & 1.48 & 0.247733 & 1.35 & 0.83 & 1907 & 11 & 1632 & 12 & 1427 & 17.25 & 74.83 \\
\hline z21 & 0.13 & 54494 & 0.121427 & 0.59 & 4.743 & 1.36 & 0.283302 & 1.23 & 0.82 & 1977 & 11 & 1775 & 11 & 1608 & 17.46 & 81.32 \\
\hline RS-234 & & & & & & & & & & & & & & & & \\
\hline z01 & 0.03 & 18134 & 0.084467 & 0.91 & 1.528 & 1.65 & 0.131159 & 1.38 & 0.67 & 1303 & 18 & 941 & 10 & 794 & 10.31 & 60.96 \\
\hline z02 & 0.18 & 13927 & 0.130665 & 0.82 & 6.313 & 1.85 & 0.350420 & 1.65 & 0.74 & 2107 & 14 & 2020 & 16 & 1937 & 27.67 & 91.92 \\
\hline Z03 & 0.13 & 16721 & 0.122891 & 0.85 & 4.356 & 1.93 & 0.257055 & 1.73 & 0.86 & 1999 & 15 & 1704 & 16 & 1475 & 22.78 & 73.79 \\
\hline Z04 & 0.14 & 16151 & 0.127211 & 1.29 & 5.418 & 3.79 & 0.308895 & 3.56 & 0.92 & 2060 & 23 & 1888 & 32 & 1735 & 54.15 & 84.24 \\
\hline z05 & 0.22 & 27183 & 0.135319 & 0.69 & 7.018 & 1.47 & 0.376155 & 1.29 & 0.74 & 2168 & 12 & 2114 & 13 & 2058 & 22.79 & 94.93 \\
\hline z06core & 0.17 & 17595 & 0.134927 & 0.68 & 7.367 & 1.74 & 0.395973 & 1.60 & 0.79 & 2163 & 12 & 2157 & 16 & 2150 & 29.21 & 99.42 \\
\hline z07 & 0.26 & 25885 & 0.135052 & 0.57 & 7.320 & 1.19 & 0.393100 & 1.05 & 0.74 & 2165 & 10 & 2151 & 11 & 2137 & 19.13 & 98.73 \\
\hline Z08 & 0.00 & 10511 & 0.060107 & 2.39 & 0.825 & 3.19 & 0.099505 & 2.11 & 0.66 & 607 & 52 & 611 & 15 & 612 & 12.33 & 100.67 \\
\hline z09 & 0.24 & 34216 & 0.136604 & 0.60 & 7.470 & 1.59 & 0.396593 & 1.47 & 0.81 & 2185 & 11 & 2169 & 14 & 2153 & 26.96 & 98.57 \\
\hline $\mathrm{z} 10$ & 0.24 & 20835 & 0.136052 & 0.66 & 7.647 & 1.16 & 0.407654 & 0.95 & 0.67 & 2178 & 11 & 2190 & 10 & 2204 & 17.73 & 101.22 \\
\hline z11 & 0.20 & 18295 & 0.135226 & 0.76 & 7.311 & 1.55 & 0.392137 & 1.35 & 0.71 & 2167 & 13 & 2150 & 14 & 2133 & 24.60 & 98.42 \\
\hline z13 & 0.07 & 20749 & 0.114581 & 1.02 & 3.241 & 2.16 & 0.205160 & 1.91 & 0.72 & 1873 & 18 & 1467 & 17 & 1203 & 20.96 & 64.22 \\
\hline z14 & 0.22 & 21966 & 0.136601 & 0.61 & 7.233 & 1.32 & 0.384043 & 1.18 & 0.77 & 2185 & 11 & 2141 & 12 & 2095 & 21.02 & 95.91 \\
\hline Z15 & 0.15 & 44167 & 0.131900 & 0.96 & 5.909 & 2.05 & 0.324918 & 1.81 & 0.85 & 2123 & 17 & 1963 & 18 & 1814 & 28.65 & 85.41 \\
\hline z16 & 0.23 & 37576 & 0.136330 & 0.61 & 7.331 & 1.42 & 0.390023 & 1.28 & 0.81 & 2181 & 11 & 2153 & 13 & 2123 & 23.13 & 97.33 \\
\hline z17 & 0.23 & 239834 & 0.133987 & 0.65 & 6.975 & 1.35 & 0.377571 & 1.18 & 0.77 & 2151 & 11 & 2108 & 12 & 2065 & 20.87 & 96.00 \\
\hline z18 & 0.17 & 27955 & 0.134083 & 0.57 & 7.058 & 1.33 & 0.381771 & 1.20 & 0.80 & 2152 & 10 & 2119 & 12 & 2085 & 21.38 & 96.86 \\
\hline Z19 & 0.17 & 34123 & 0.125644 & 0.97 & 5.114 & 1.92 & 0.295208 & 1.66 & 0.84 & 2038 & 17 & 1838 & 16 & 1668 & 24.39 & 81.83 \\
\hline $\mathrm{z} 20$ & 0.17 & 24058 & 0.129251 & 0.74 & 5.471 & 1.52 & 0.306995 & 1.33 & 0.78 & 2088 & 13 & 1896 & 13 & 1726 & 20.15 & 82.67 \\
\hline z21 & 0.20 & 169154 & 0.135750 & 0.64 & 7.126 & 1.40 & 0.380706 & 1.25 & 0.80 & 2174 & 11 & 2127 & 12 & 2080 & 22.16 & 95.67 \\
\hline z22 & 0.17 & 29712 & 0.134061 & 0.72 & 6.969 & 1.36 & 0.377000 & 1.15 & 0.73 & 2152 & 13 & 2107 & 12 & 2062 & 20.30 & 95.84 \\
\hline Z23 & 0.16 & 20188 & 0.134826 & 1.20 & 7.180 & 2.64 & 0.386256 & 2.35 & 0.87 & 2162 & 21 & 2134 & 24 & 2105 & 42.26 & 97.39 \\
\hline z24 & 0.20 & 48806 & 0.133861 & 0.61 & 6.586 & 1.26 & 0.356814 & 1.10 & 0.76 & 2149 & 11 & 2057 & 11 & 1967 & 18.70 & 91.52 \\
\hline z25 & 0.17 & 31060 & 0.129885 & 0.62 & 5.456 & 1.23 & 0.304667 & 1.06 & 0.75 & 2096 & 11 & 1894 & 11 & 1714 & 15.92 & 81.78 \\
\hline z26 & 0.02 & 9972 & 0.091555 & 1.11 & 1.766 & 1.89 & 0.139904 & 1.53 & 0.64 & 1458 & 21 & 1033 & 12 & 844 & 12.10 & 57.89 \\
\hline z27 & 0.27 & 42787 & 0.135533 & 0.68 & 7.062 & 1.42 & 0.377902 & 1.25 & 0.80 & 2171 & 12 & 2119 & 13 & 2066 & 22.07 & 95.19 \\
\hline $\begin{array}{l}\text { RS-423 } \\
\text { larger zi }\end{array}$ & $\begin{array}{l}\text { thoam } \\
\text { ons }\end{array}$ & ibolite) & & & & & & & & & & & & & & \\
\hline z04 & 0.44 & 10016 & 0.125843 & 1.04 & 6.405 & 4.00 & 0.369114 & 3.86 & 0.97 & 2041 & 18 & 2033 & 35 & 2025 & 67 & 99.24 \\
\hline z06 & 0.49 & 99569 & 126777 & 0.57 & 6.320 & 1.23 & 0.361540 & 1.09 & 0.88 & 2054 & 10 & 2021 & 11 & 1989 & 19 & - \\
\hline
\end{tabular}


Table 1 (continued)

\begin{tabular}{|c|c|c|c|c|c|c|c|c|c|c|c|c|c|c|c|c|}
\hline \multirow[b]{2}{*}{ Samples } & \multirow[t]{2}{*}{$\mathrm{Th} / \mathrm{U}$} & \multicolumn{7}{|l|}{ Ratios } & \multirow[t]{3}{*}{$\rho$} & Appare & t ages & & & & & Conc. (\%) \\
\hline & & $206 \mathrm{~Pb}$ & $207 \mathrm{~Pb}$ & $\pm 1 \sigma$ & $207 \mathrm{~Pb}$ & $\pm 1 \sigma$ & $206 \mathrm{~Pb}$ & $\pm 1 \sigma$ & & $207 \mathrm{~Pb}$ & (Ma) & $207 \mathrm{~Pb}$ & (Ma) & $206 \mathrm{~Pb}$ & (Ma) & \\
\hline Spots & & $204 \mathrm{~Pb}$ & $206 \mathrm{~Pb}$ & & $235 \mathrm{U}$ & & $238 \mathrm{U}$ & & & $206 \mathrm{~Pb}$ & & $235 \mathrm{U}$ & & $238 \mathrm{U}$ & & \\
\hline z07 & 0.44 & 6229 & 0.126679 & 1.05 & 6.509 & 3.95 & 0.372627 & 3.81 & 0.96 & 2052 & 19 & 2047 & 35 & 2042 & 67 & 99.48 \\
\hline Z08 & 0.43 & 18393 & 0.125677 & 0.83 & 6.450 & 2.18 & 0.372214 & 2.01 & 0.92 & 2038 & 15 & 2039 & 19 & 2040 & 35 & 100.07 \\
\hline z09 & 0.30 & 17032 & 0.124685 & 0.74 & 6.319 & 1.86 & 0.367588 & 1.71 & 0.91 & 2024 & 13 & 2021 & 16 & 2018 & 30 & 99.69 \\
\hline $\mathrm{z} 10$ & 0.36 & 25808 & 0.125905 & 0.92 & 6.158 & 1.80 & 0.354743 & 1.54 & 0.93 & 2042 & 16 & 1999 & 16 & 1957 & 26 & 95.87 \\
\hline z01 & 0.31 & 4565 & 0.124898 & 1.45 & 5.897 & 6.21 & 0.342419 & 6.02 & 0.97 & 2027 & 25 & 1961 & 53 & 1898 & 99 & 93.63 \\
\hline Z02 & 0.23 & 2135 & 0.128024 & 1.58 & 6.048 & 4.76 & 0.342639 & 4.47 & 0.94 & 2071 & 28 & 1983 & 41 & 1899 & 73 & 91.71 \\
\hline small zirc & ns & & & & & & & & & & & & & & & \\
\hline $\mathrm{Z} 11$ & 0.09 & 10495 & 0.123309 & 0.85 & 5.736 & 2.67 & 0.337345 & 2.52 & 0.95 & 2005 & 15 & 1937 & 23 & 1874 & 41 & 93.48 \\
\hline $\mathrm{Z} 14$ & 0.14 & 14536 & 0.123150 & 0.84 & 5.684 & 2.13 & 0.334766 & 1.95 & 0.92 & 2002 & 15 & 1929 & 18 & 1861 & 32 & 92.96 \\
\hline $\mathrm{z} 16$ & 0.10 & 12335 & 0.122028 & 0.80 & 6.112 & 3.40 & 0.363238 & 3.30 & 0.97 & 1986 & 14 & 1992 & 29 & 1998 & 57 & 100.58 \\
\hline Z18 & 0.06 & 14086 & 0.121585 & 0.82 & 5.697 & 2.65 & 0.339842 & 2.51 & 0.95 & 1980 & 15 & 1931 & 23 & 1886 & 41 & 95.27 \\
\hline z19 & 0.08 & 5497 & 0.123663 & 0.87 & 5.217 & 3.24 & 0.305984 & 3.11 & 0.96 & 2010 & 15 & 1855 & 27 & 1721 & 47 & 85.63 \\
\hline $\mathrm{z} 12$ & 0.04 & 5043 & 0.134641 & 1.10 & 7.554 & 5.03 & 0.406917 & 4.91 & 0.98 & 2159 & 19 & 2179 & 45 & 2201 & 92 & 101.92 \\
\hline z13 & 0.09 & 3490 & 0.127969 & 3.40 & 5.877 & 12.38 & 0.333097 & 11.85 & 0.99 & 2070 & 59 & 1958 & 102 & 1853 & 189 & 89.52 \\
\hline Z15 & 0.04 & 4217 & 0.127501 & 1.56 & 6.675 & 6.17 & 0.379707 & 5.94 & 0.97 & 2064 & 27 & 2069 & 53 & 2075 & 105 & 100.54 \\
\hline Z17 & 0.18 & 4591 & 0.161203 & 1.45 & 8.863 & 8.64 & 0.398749 & 8.49 & 0.99 & 2468 & 24 & 2324 & 76 & 2163 & 155 & 87.64 \\
\hline GL-479 & ăo João & inho) & & & & & & & & & & & & & & \\
\hline z01 & 0.13 & 50617 & 0.121702 & 0.97 & 4.638 & 1.40 & 0.276404 & 1.02 & 0.68 & 1981 & 17 & 1756 & 12 & 1573 & 14.18 & 79.40 \\
\hline z02core & 0.14 & 44173 & 0.125627 & 0.87 & 5.470 & 1.37 & 0.315764 & 1.07 & 0.69 & 2038 & 15 & 1896 & 12 & 1769 & 16.51 & 86.82 \\
\hline z03 & 0.13 & 12385 & 0.131039 & 0.98 & 7.504 & 2.40 & 0.415314 & 2.20 & 0.79 & 2112 & 17 & 2173 & 22 & 2239 & 41.55 & 106.02 \\
\hline z04 & 0.14 & 74015 & 0.129503 & 0.69 & 6.447 & 1.24 & 0.361042 & 1.03 & 0.72 & 2091 & 12 & 2039 & 11 & 1987 & 17.62 & 95.02 \\
\hline z05core & 0.17 & 41453 & 0.128952 & 0.65 & 6.196 & 1.24 & 0.348501 & 1.05 & 0.73 & 2084 & 11 & 2004 & 11 & 1927 & 17.53 & 92.50 \\
\hline z06 & 0.24 & 40705 & 0.130336 & 1.06 & 6.818 & 1.59 & 0.379406 & 1.19 & 0.71 & 2102 & 19 & 2088 & 14 & 2074 & 21.15 & 98.62 \\
\hline z07 & 0.14 & 16301 & 0.129984 & 0.85 & 6.583 & 1.73 & 0.367291 & 1.51 & 0.72 & 2098 & 15 & 2057 & 15 & 2017 & 26.10 & 96.13 \\
\hline z08 & 0.31 & 23807 & 0.130951 & 0.77 & 6.864 & 1.64 & 0.380177 & 1.45 & 0.73 & 2111 & 14 & 2094 & 15 & 2077 & 25.72 & 98.41 \\
\hline z09 & 0.18 & 16680 & 0.127232 & 0.68 & 5.627 & 1.72 & 0.320775 & 1.58 & 0.80 & 2060 & 12 & 1920 & 15 & 1794 & 24.76 & 87.06 \\
\hline $\mathrm{z} 10$ & 0.23 & 8248 & 0.127089 & 1.71 & 5.737 & 2.84 & 0.327422 & 2.27 & 0.80 & 2058 & 30 & 1937 & 25 & 1826 & 36.13 & 88.72 \\
\hline $\mathrm{z} 11$ & 0.10 & 15709 & 0.130906 & 0.83 & 6.581 & 1.89 & 0.364625 & 1.69 & 0.78 & 2110 & 14 & 2057 & 17 & 2004 & 29.19 & 94.97 \\
\hline $\mathrm{z} 12$ & 0.14 & 39018 & 0.121411 & 0.85 & 5.151 & 1.81 & 0.307685 & 1.60 & 0.75 & 1977 & 15 & 1845 & 15 & 1729 & 24.22 & 87.47 \\
\hline $\mathrm{z} 13$ & 0.25 & 37516 & 0.131081 & 0.60 & 6.860 & 1.34 & 0.379545 & 1.20 & 0.80 & 2112 & 11 & 2093 & 12 & 2074 & 21.27 & 98.19 \\
\hline $\mathrm{z} 14$ & 0.32 & 18532 & 0.128757 & 1.00 & 6.216 & 1.96 & 0.350155 & 1.69 & 0.73 & 2081 & 18 & 2007 & 17 & 1935 & 28.22 & 93.00 \\
\hline $\mathrm{z} 15$ & 0.29 & 12685 & 0.129475 & 1.00 & 6.901 & 2.38 & 0.386579 & 2.16 & 0.78 & 2091 & 18 & 2099 & 21 & 2107 & 38.91 & 100.77 \\
\hline z16rim & 0.10 & 16344 & 0.128660 & 1.50 & 6.402 & 2.23 & 0.360859 & 1.65 & 0.72 & 2080 & 26 & 2032 & 20 & 1986 & 28.22 & 95.50 \\
\hline z16core & 0.17 & 23628 & 0.123737 & 0.88 & 5.636 & 2.19 & 0.330344 & 2.00 & 0.80 & 2011 & 16 & 1922 & 19 & 1840 & 32.07 & 91.51 \\
\hline $\mathrm{z} 17$ & 0.27 & 128880 & 0.121907 & 1.08 & 4.708 & 1.78 & 0.280092 & 1.42 & 0.70 & 1984 & 19 & 1769 & 15 & 1592 & 20.00 & 80.22 \\
\hline $\mathrm{z} 18$ & 0.23 & 22712 & 0.129436 & 0.94 & 7.014 & 2.09 & 0.392987 & 1.87 & 0.78 & 2090 & 17 & 2113 & 19 & 2137 & 33.96 & 102.22 \\
\hline z19 & 0.15 & 12669 & 0.129679 & 0.94 & 7.024 & 2.05 & 0.392828 & 1.82 & 0.77 & 2094 & 17 & 2114 & 18 & 2136 & 33.09 & 102.02 \\
\hline $\mathrm{z} 20$ & 0.16 & 18897 & 0.125094 & 1.32 & 5.673 & 1.86 & 0.328884 & 1.31 & 0.71 & 2030 & 23 & 1927 & 16 & 1833 & 20.87 & 90.29 \\
\hline SU-541 & ertente & & & & & & & & & & & & & & & \\
\hline 003-Z01 & 0.22 & 1255508 & 0.12136 & 0.90 & 5.9510 & 1.70 & 0.35565 & 1.44 & 0.84 & 1976 & 16 & 1969 & 15 & 1962 & 24.38 & 99.25 \\
\hline 004-Z02 & 0.18 & 1200 & 0.13846 & 1.45 & 7.4169 & 1.90 & 0.38849 & 1.22 & 0.63 & 2208 & 25 & 2163 & 17 & 2116 & 22.00 & 95.82 \\
\hline 005-Z03 & 0.41 & 3867 & 0.14059 & 0.72 & 8.2573 & 1.29 & 0.42596 & 1.07 & 0.82 & 2235 & 12 & 2260 & 12 & 2287 & 20.55 & 102.37 \\
\hline 006-Z04 & 0.12 & 538398 & 0.12664 & 0.60 & 6.9037 & 1.07 & 0.39538 & 0.88 & 0.86 & 2052 & 11 & 2099 & 9 & 2148 & 16.12 & 104.67 \\
\hline 009-Z05 & 0.40 & 325069 & 0.12952 & 0.54 & 7.3045 & 1.15 & 0.40903 & 1.01 & 0.87 & 2091 & 10 & 2149 & 10 & 2211 & 18.90 & 105.69 \\
\hline 010-Z06 & 0.32 & 2654 & 0.13821 & 0.86 & 6.6659 & 1.71 & 0.34980 & 1.47 & 0.86 & 2205 & 15 & 2068 & 15 & 1934 & 24.68 & 87.70 \\
\hline 011-Z07 & 0.17 & 96580 & 0.12355 & 0.48 & 6.3203 & 1.54 & 0.37101 & 1.47 & 0.95 & 2008 & 9 & 2021 & 14 & 2034 & 25.56 & 101.30 \\
\hline 012-Z08 & 0.39 & 466014 & 0.12633 & 0.82 & 7.3708 & 1.34 & 0.42315 & 1.07 & 0.88 & 2048 & 14 & 2157 & 12 & 2275 & 20.42 & 111.09 \\
\hline 015-Z09 & 0.21 & 32911 & 0.12690 & 0.62 & 5.7724 & 2.82 & 0.32990 & 2.75 & 0.98 & 2056 & 11 & 1942 & 24 & 1838 & 43.80 & 89.41 \\
\hline 016-Z10 & 0.19 & 32312 & 0.12717 & 0.50 & 6.1263 & 1.23 & 0.34940 & 1.12 & 0.91 & 2059 & 9 & 1994 & 11 & 1932 & 18.74 & 93.81 \\
\hline $017-Z 11$ & 0.22 & 220748 & 0.12359 & 0.50 & 5.3877 & 0.94 & 0.31617 & 0.79 & 0.82 & 2009 & 9 & 1883 & 8 & 1771 & 12.25 & 88.17 \\
\hline 018-Z12 & 0.59 & 45590 & 0.12310 & 0.50 & 6.4119 & 1.44 & 0.37777 & 1.35 & 0.96 & 2002 & 9 & 2034 & 13 & 2066 & 23.84 & 103.21 \\
\hline $021-Z 13$ & 0.31 & 197888 & 0.12622 & 1.80 & 5.6470 & 2.36 & 0.32448 & 1.53 & 0.64 & 2046 & 31 & 1923 & 20 & 1812 & 24.14 & 88.54 \\
\hline $022-Z 14$ & 0.24 & 95112 & 0.12771 & 0.44 & 7.2659 & 1.00 & 0.41263 & 0.90 & 0.89 & 2067 & 8 & 2145 & 9 & 2227 & 16.96 & 107.75 \\
\hline 023-Z15 & 0.10 & 1450340 & 0.12770 & 0.47 & 7.1922 & 1.60 & 0.40847 & 1.53 & 0.95 & 2067 & 8 & 2136 & 14 & 2208 & 28.64 & 106.84 \\
\hline 027-Z16 & 0.29 & 1566940 & 0.12680 & 0.64 & 5.8995 & 1.35 & 0.33743 & 1.19 & 0.87 & 2054 & 11 & 1961 & 12 & 1874 & 19.31 & 91.25 \\
\hline 028-Z17 & 0.15 & 408838 & 0.12017 & 1.91 & 6.0963 & 2.35 & 0.36793 & 1.36 & 0.57 & 1959 & 34 & 1990 & 20 & 2020 & 23.58 & 103.11 \\
\hline 029-Z18 & 0.06 & 319407 & 0.11986 & 0.91 & 5.6091 & 1.78 & 0.33941 & 1.53 & 0.85 & 1954 & 16 & 1918 & 15 & 1884 & 24.94 & 96.40 \\
\hline 030-z19 & 0.24 & 374102 & 0.13487 & 7.61 & 7.9474 & 11.18 & 0.42737 & 8.19 & 0.91 & 2162 & 133 & 2225 & 101 & 2294 & 157.99 & 106.08 \\
\hline 033-Z20 & 0.12 & 3790 & 0.14488 & 1.47 & 6.9861 & 1.81 & 0.34972 & 1.04 & 0.56 & 2286 & 25 & 2110 & 16 & 1933 & 17.46 & 84.56 \\
\hline 034-Z21 & 0.21 & 60268 & 0.12945 & 0.46 & 7.4638 & 1.79 & 0.41818 & 1.72 & 0.96 & 2090 & 8 & 2169 & 16 & 2252 & 32.77 & 107.74 \\
\hline 035-Z22 & 0.60 & 298764 & 0.12779 & 0.49 & 6.9507 & 1.38 & 0.39449 & 1.29 & 0.93 & 2068 & 9 & 2105 & 12 & 2144 & 23.59 & 103.67 \\
\hline $036-z 23$ & 0.22 & 246934 & 0.12036 & 0.71 & 6.4773 & 1.33 & 0.39033 & 1.13 & 0.92 & 1962 & 13 & 2043 & 12 & 2124 & 20.40 & 108.30 \\
\hline 039-Z24 & 0.08 & 211025 & 0.12366 & 0.65 & 6.5183 & 1.27 & 0.38230 & 1.09 & 0.85 & 2010 & 12 & 2048 & 11 & 2087 & 19.47 & 103.84 \\
\hline $040-Z 25$ & 0.32 & 258598 & 0.13028 & 0.47 & 7.3547 & 1.01 & 0.40942 & 0.90 & 0.87 & 2102 & 8 & 2155 & 9 & 2212 & 16.78 & 105.26 \\
\hline $041-Z 26$ & 0.42 & 443388 & 0.13134 & 0.54 & 7.3288 & 1.47 & 0.40469 & 1.37 & 0.93 & 2116 & 9 & 2152 & 13 & 2191 & 25.41 & 103.52 \\
\hline $042-z 27$ & 0.28 & 6657 & 0.12898 & 1.88 & 6.7247 & 2.25 & 0.37814 & 1.23 & 0.77 & 2084 & 33 & 2076 & 20 & 2068 & 21.74 & 99.21 \\
\hline SU-515 & lsic gn & & & & & & & & & & & & & & & \\
\hline $047-Z 30$ & 0.07 & 212 & 0.07016 & 26.72 & 0.7880 & 27.07 & 0.08146 & 4.36 & 0.31 & 933 & 548 & 590 & 121 & 505 & 21.18 & 54.11 \\
\hline 018 Z12 & 0.07 & 41174 & 0.10937 & 1.73 & 5.670476 & 2.40 & 0.37602 & 1.67 & 0.72 & 1789 & 31 & 1927 & 21 & 2058 & 29.34 & 115.02 \\
\hline 025 Z16 & 0.09 & 250 & 0.09282 & 5.04 & 1.629833 & 5.34 & 0.12735 & 1.76 & 0.33 & 1484 & 95 & 982 & 34 & 773 & 12.81 & 52.06 \\
\hline 056-Z35 & 0.09 & 1971 & 0.12964 & 1.34 & 6.9918 & 1.67 & 0.39117 & 0.99 & 0.57 & 2093 & 24 & 2110 & 15 & 2128 & 17.98 & 101.68 \\
\hline
\end{tabular}


Table 1 (continued)

\begin{tabular}{|c|c|c|c|c|c|c|c|c|c|c|c|c|c|c|c|c|}
\hline \multirow[b]{2}{*}{ Samples } & \multirow[t]{3}{*}{$\mathrm{Th} / \mathrm{U}$} & \multicolumn{7}{|l|}{ Ratios } & \multirow[t]{3}{*}{$\rho$} & \multicolumn{6}{|c|}{ Apparent ages } & \multirow[t]{3}{*}{ Conc. (\%) } \\
\hline & & $206 \mathrm{~Pb}$ & $207 \mathrm{~Pb}$ & $\pm 1 \sigma$ & $207 \mathrm{~Pb}$ & $\pm 1 \sigma$ & $206 \mathrm{~Pb}$ & $\pm 1 \sigma$ & & $207 \mathrm{~Pb}$ & (Ma) & $207 \mathrm{~Pb}$ & (Ma) & $206 \mathrm{~Pb}$ & (Ma) & \\
\hline Spots & & $204 \mathrm{~Pb}$ & $206 \mathrm{~Pb}$ & & $235 U$ & & $238 \mathrm{U}$ & & & $206 \mathrm{~Pb}$ & & $235 U$ & & $238 U$ & & \\
\hline 019 Z13 & 0.09 & 133867 & 0.10899 & 1.49 & 5.193905 & 3.05 & 0.34563 & 2.66 & 0.87 & 1783 & 27 & 1852 & 26 & 1914 & 44.08 & 107.35 \\
\hline 058-Z37 & 0.12 & 2258 & 0.11440 & 2.15 & 5.9778 & 2.44 & 0.37899 & 1.17 & 0.46 & 1870 & 39 & 1973 & 21 & 2072 & 20.67 & 110.76 \\
\hline 036 Z25 & 0.12 & 42311 & 0.11527 & 1.12 & 5.873405 & 2.08 & 0.36956 & 1.76 & 0.84 & 1884 & 20 & 1957 & 18 & 2027 & 30.57 & 107.61 \\
\hline 045-Z28 & 0.12 & 59 & 0.13273 & 1.29 & 5.9634 & 6.30 & 0.32586 & 4.65 & 0.96 & 2134 & 22 & 1971 & 53 & 1818 & 96.97 & 85.19 \\
\hline 030 Z21 & 0.16 & 21496 & 0.12486 & 1.07 & 6.805503 & 2.03 & 0.39531 & 1.72 & 0.85 & 2027 & 19 & 2086 & 18 & 2147 & 31.45 & 105.95 \\
\hline $031 \mathrm{Z22}$ & 0.16 & 61078 & 0.11792 & 1.26 & 5.789927 & 2.19 & 0.35610 & 1.79 & 0.81 & 1925 & 22 & 1945 & 19 & 1964 & 30.32 & 102.01 \\
\hline 015 Z09 & 0.18 & 30452 & 0.11822 & 1.72 & 6.668465 & 2.22 & 0.40911 & 1.40 & 0.62 & 1929 & 31 & 2068 & 20 & 2211 & 26.17 & 114.58 \\
\hline 053-Z34 & 0.18 & 506766 & 0.12151 & 2.10 & 6.6542 & 2.26 & 0.39719 & 0.83 & 0.58 & 1978 & 37 & 2067 & 20 & 2156 & 15.24 & 108.98 \\
\hline 029 Z20 & 0.19 & 10001 & 0.12150 & 1.87 & 6.148167 & 3.17 & 0.36700 & 2.57 & 0.81 & 1978 & 33 & 1997 & 28 & 2015 & 44.42 & 101.87 \\
\hline 035 Z24 & 0.22 & 95374 & 0.11275 & 1.02 & 6.987447 & 1.63 & 0.44945 & 1.28 & 0.77 & 1844 & 18 & 2110 & 15 & 2393 & 25.49 & 129.74 \\
\hline 046-Z29 & 0.25 & 250 & 0.13438 & 1.12 & 7.2590 & 1.52 & 0.39177 & 0.96 & 0.63 & 2156 & 19 & 2144 & 13 & 2131 & 18.52 & 98.84 \\
\hline 032 Z23 & 0.26 & 11007 & 0.13060 & 1.94 & 6.985509 & 3.22 & 0.38793 & 2.57 & 0.84 & 2106 & 34 & 2110 & 28 & 2113 & 46.22 & 100.34 \\
\hline 004 Z02 & 0.26 & 16004 & 0.12237 & 1.06 & 6.568921 & 1.96 & 0.38934 & 1.64 & 0.84 & 1991 & 19 & 2055 & 17 & 2120 & 29.65 & 106.46 \\
\hline 026 Z17 & 0.28 & 6252 & 0.12258 & 3.13 & 6.278343 & 4.20 & 0.37147 & 2.78 & 0.66 & 1994 & 55 & 2015 & 36 & 2036 & 48.72 & 102.12 \\
\hline 009 Z05 & 0.30 & 25671 & 0.09598 & 1.20 & 2.370679 & 2.60 & 0.17914 & 2.30 & 0.88 & 1547 & 22 & 1234 & 18 & 1062 & 22.54 & 68.65 \\
\hline 020 Z14 & 0.31 & 23650 & 0.10750 & 2.08 & 2.83201 & 3.58 & 0.19106 & 2.91 & 0.81 & 1758 & 38 & 1364 & 27 & 1127 & 30.04 & 64.13 \\
\hline 012 Z08 & 0.31 & 15868 & 0.11332 & 3.13 & 6.439738 & 3.99 & 0.41217 & 2.47 & 0.75 & 1853 & 57 & 2038 & 35 & 2225 & 46.56 & 120.05 \\
\hline 028 Z19 & 0.32 & 10693 & 0.11108 & 2.55 & 3.636655 & 3.32 & 0.23744 & 2.12 & 0.75 & 1817 & 46 & 1558 & 26 & 1373 & 26.20 & 75.57 \\
\hline 003 Z01 & 0.33 & 17919 & 0.11037 & 1.61 & 3.854067 & 5.00 & 0.25327 & 4.73 & 0.95 & 1805 & 29 & 1604 & 40 & 1455 & 61.40 & 80.61 \\
\hline $0317 Z 26$ & 0.33 & 8624 & 0.11465 & 1.43 & 3.76107 & 2.38 & 0.23792 & 1.91 & 0.80 & 1874 & 26 & 1584 & 19 & 1376 & 23.61 & 73.40 \\
\hline 044-Z27 & 0.37 & 18596 & 0.11264 & 0.93 & 3.6615 & 1.66 & 0.23576 & 1.38 & 0.82 & 1842 & 17 & 1563 & 13 & 1365 & 16.94 & 74.06 \\
\hline 059-Z38 & 0.38 & 152598 & 0.11541 & 1.63 & 4.2015 & 2.93 & 0.26404 & 2.44 & 0.94 & 1886 & 29 & 1674 & 24 & 1510 & 32.71 & 80.08 \\
\hline 005 Z03 & 0.38 & 22165 & 0.12039 & 1.32 & 6.10018 & 2.50 & 0.36748 & 2.12 & 0.85 & 1962 & 24 & 1990 & 22 & 2018 & 36.78 & 102.83 \\
\hline 006 Z04 & 0.42 & 16954 & 0.10433 & 8.91 & 5.629647 & 9.64 & 0.39135 & 3.68 & 0.62 & 1703 & 164 & 1921 & 83 & 2129 & 66.80 & 125.05 \\
\hline 016 Z10 & 0.43 & 27751 & 0.11571 & 1.79 & 5.528955 & 2.45 & 0.34655 & 1.68 & 0.68 & 1891 & 32 & 1905 & 21 & 1918 & 27.81 & 101.43 \\
\hline 027 Z18 & 0.44 & 11528 & 0.11816 & 1.37 & 5.370307 & 2.51 & 0.32963 & 2.10 & 0.84 & 1929 & 24 & 1880 & 21 & 1837 & 33.59 & 95.23 \\
\hline 057-Z36 & 0.44 & 3687 & 0.12897 & 1.43 & 6.2149 & 3.71 & 0.34948 & 3.41 & 0.92 & 2084 & 25 & 2007 & 32 & 1932 & 56.85 & 92.71 \\
\hline 010 Z06 & 0.47 & 8534 & 0.07261 & 9.34 & 3.28981 & 9.49 & 0.32859 & 1.71 & 0.18 & 1003 & 190 & 1479 & 74 & 1832 & 27.28 & 182.58 \\
\hline 051-Z32 & 0.53 & 2506 & 0.10103 & 1.15 & 3.3320 & 1.83 & 0.23920 & 1.41 & 0.77 & 1643 & 21 & 1489 & 14 & 1383 & 17.67 & 84.14 \\
\hline 011 Z07 & 0.56 & 431 & 0.09392 & 5.12 & 3.250166 & 8.51 & 0.25098 & 6.55 & 0.79 & 1507 & 94 & 1469 & 64 & 1444 & 87.36 & 95.82 \\
\hline 050-Z31 & 0.63 & 150 & 0.05942 & 7.97 & 1.0462 & 9.82 & 0.12769 & 5.08 & 0.54 & 583 & 164 & 727 & 50 & 775 & 41.75 & 132.96 \\
\hline 017 Z11 & 0.65 & 11809 & 0.11459 & 1.83 & 6.185003 & 2.79 & 0.39147 & 2.11 & 0.75 & 1873 & 33 & 2002 & 24 & 2130 & 38.20 & 113.67 \\
\hline 052-Z33 & 0.85 & 85 & 0.11046 & 1.83 & 1.5926 & 4.68 & 0.10457 & 3.42 & 0.88 & 1807 & 33 & 967 & 29 & 641 & 26.20 & 35.48 \\
\hline
\end{tabular}

weighted mean age of $1991 \pm 5 \mathrm{Ma}$, which suggests that this magmatism is the youngest in the studied area.

\section{U-Pb geochronology and Nd isotopes}

\subsection{Methods}

Zircons were separated using conventional techniques. After crushing and sieving of the powdered samples, heavy minerals were concentrated by panning and then by heavy liquids. The heavy mineral concentrates were subsequently processed by magnetic separation on a Frantz separator. The grains were then mounted on adhesive tape and enclosed in epoxy resin. After 1-2 days of drying, the mounts were removed from the tape and polished to about half of their thickness. $\mathrm{U}-\mathrm{Pb}$ zircon ages were obtained at the Universidade de Brasília, Brazil, using a Thermo Finningan Neptune multi-collector ICP-MS attached to a New Wave $213 \mu \mathrm{m}$ Nd-YAG solid state laser. Analytical procedures are detailed by Buhn et al. (2009). Ages were calculated using the Isoplot program of Ludwig (2000) and quoted with 95\% confidence intervals. Errors for single analysis and mean ages are quoted, respectively, at the $1 \sigma$ and $2 \sigma$ levels.

Sm-Nd analysis followed the method described by Gióia and Pimentel (2000). Whole-rock powders (ca. $50 \mathrm{mg}$ ) and leached minerals were mixed with ${ }^{149} \mathrm{Sm}-{ }^{150} \mathrm{Nd}$ spike solution and dissolved in savillex capsules. Sm and Nd extraction of whole-rock samples followed conventional cation exchange techniques, using Teflon columns containing LN-Spec resin (HDEHP - diethylhexil phosphoric acid supported on PTFE powder). Sm and Nd samples were loaded on Re evaporation of double filament assemblies and the isotopic measurements were carried out on a multi-collector Finnigan MAT 262 mass spectrometer in static mode.

Uncertainties of $\mathrm{Sm} / \mathrm{Nd}$ and ${ }^{143} \mathrm{Nd} /{ }^{144} \mathrm{Nd}$ ratios are better than $\pm 0.4 \%(1 \sigma)$ and $\pm 0.005 \%(1 \sigma)$ respectively, based on repeated analyses of international rock standards BHVO-1 and BCR-1. ${ }^{143} \mathrm{Nd} /{ }^{144} \mathrm{Nd}$ ratios were normalized to ${ }^{146} \mathrm{Nd} /{ }^{144} \mathrm{Nd}$ of 0.7219 and the decay constant used was $6.54 \times 10^{-12}$. $\mathrm{T}_{\mathrm{DM}}$ model ages values were calculated using the DePaolo (1981) model.

\subsection{Dated Samples}

Seven samples were chosen for this study. Three (SU-497, RS234 and GL-476) are from the mesosome of migmatitic orthogneisses. SU-497 was collected in the type area of the Salgadinho Complex (35 $45^{\prime} 25^{\prime \prime} \mathrm{W}, 7^{\circ} 43^{\prime} 25^{\prime \prime}$; ; Fig. 3b), RS-234 in a quarry near Vila Pará Village, Santa Cruz do Capibaribe town $\left(36^{\circ} 22^{\prime} 06^{\prime \prime} \mathrm{W}\right.$ $7^{\circ} 50^{\prime} 47^{\prime \prime S}$; Fig. 3d), and GL-476 in the Cabaceiras quarry, around Cabaceiras city $\left(36^{\circ} 18^{\prime} 11^{\prime \prime} \mathrm{W}, 7^{\circ} 28^{\prime} 59^{\prime \prime} \mathrm{S}\right.$; Fig. 3c). The fourth sample (RS-423) is from a dark green, fine- to medium-grained, granoblastic orthoamphibolite associated with granitic gneisses of the Cabaceiras unit in the northern part of the area $\left(36^{\circ} 18^{\prime} 45^{\prime \prime} \mathrm{W}\right.$, 7०31'43"S; Fig. 3e). Sample GL-479 is from an augen gneiss in the type area of São Joãozinho orthogneiss, around São Joãozinho village ( $36^{\circ} 16^{\prime} 30^{\prime \prime} \mathrm{W}, 7^{\circ} 17^{\prime} 01^{\prime \prime}$ S; Fig. 3a). Sample SU-541 is from the mafic band of a banded orthogneiss and was collected at Pedreira do Tito, c. $1.5 \mathrm{~km}$ north of the type locality of the Vertentes Complex (3553'46'"W, 742'15"S; Fig. 3f). The last sample (SU-515) is from an equigranular felsic gneiss sampled at coordinates $35^{\circ} 48^{\prime} 38^{\prime \prime} \mathrm{W}$, $7^{\circ} 44^{\prime} 39$ "S. The data obtained in this study are presented in Table 1 and plotted in Concordia diagrams on Figs. 4 and 5. 

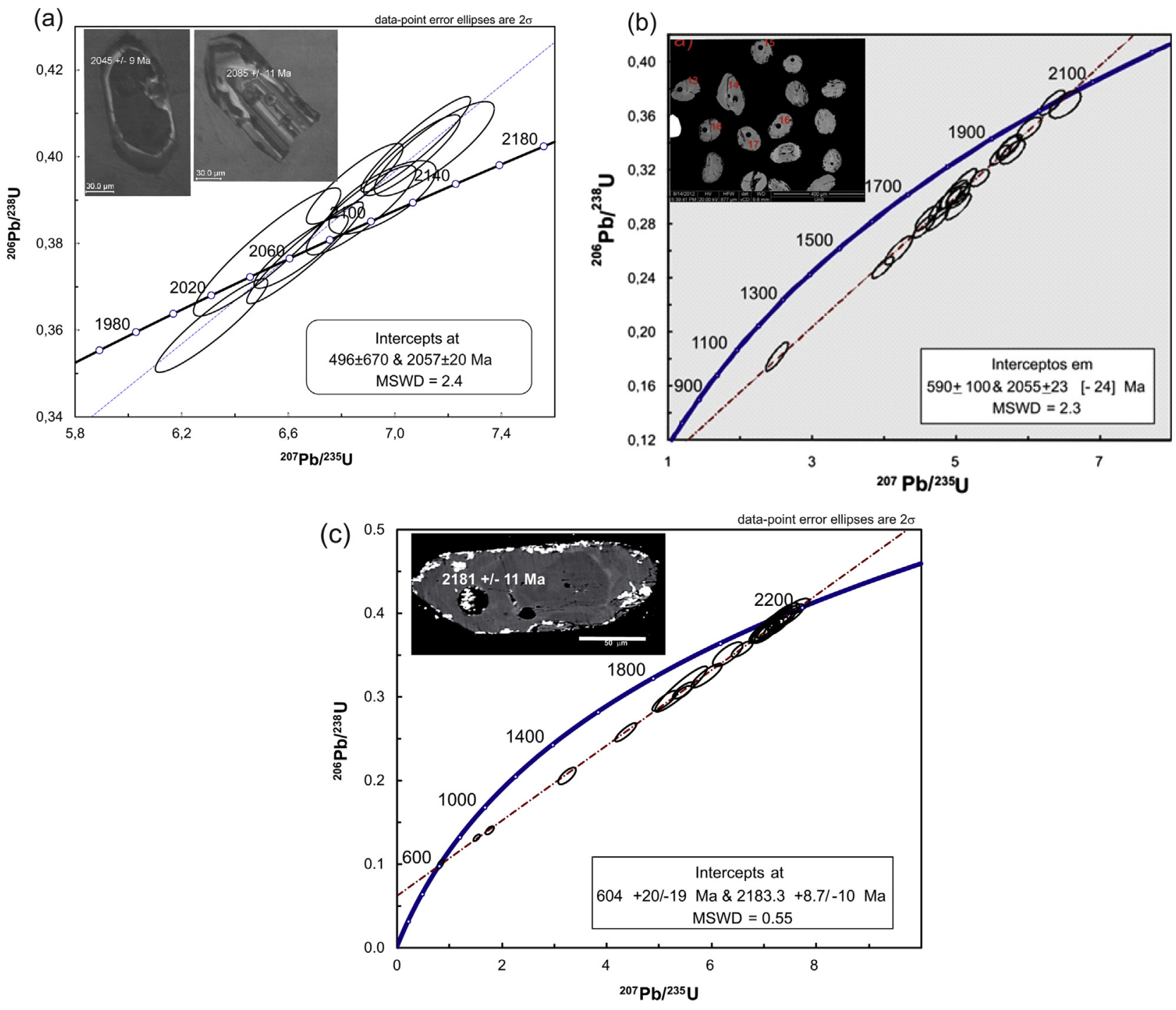

(d)
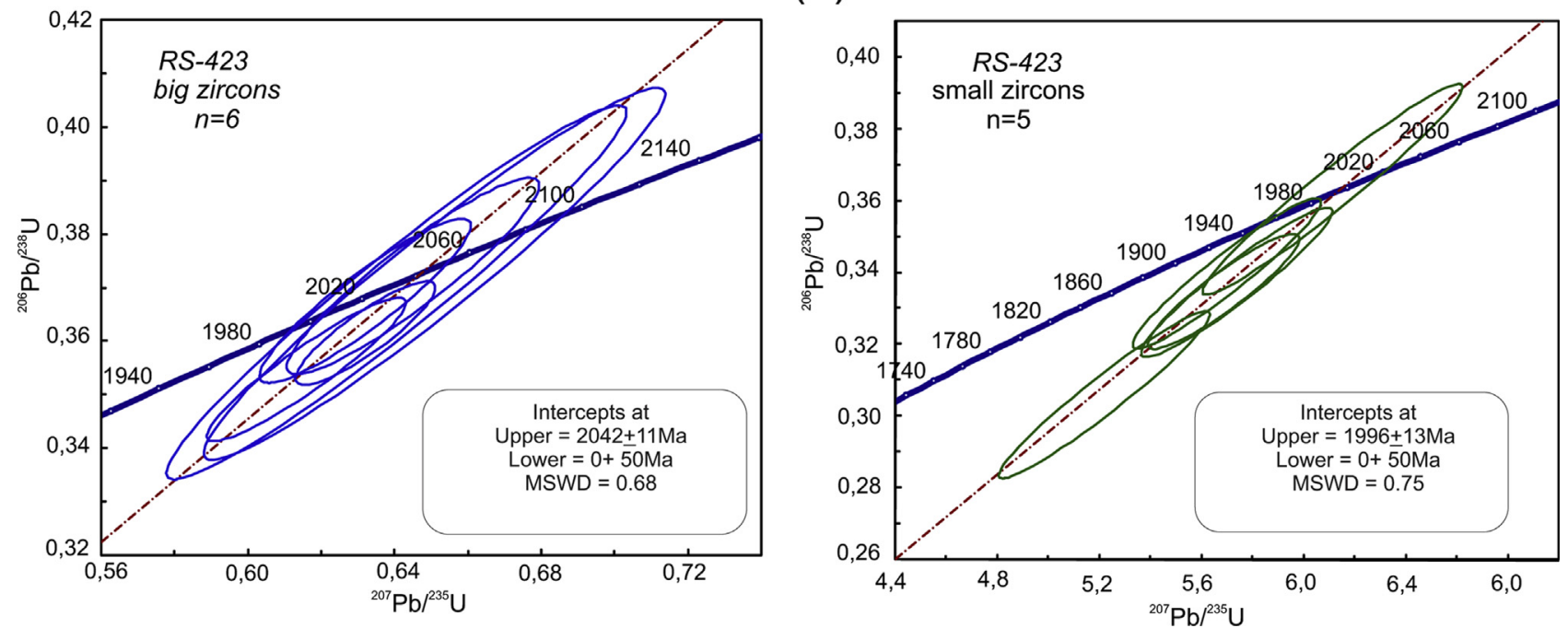

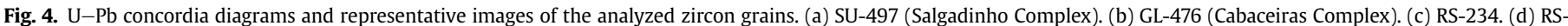
423 (orthoamphibolite). Left: large zircon grains; right: smaller zircon grains. 

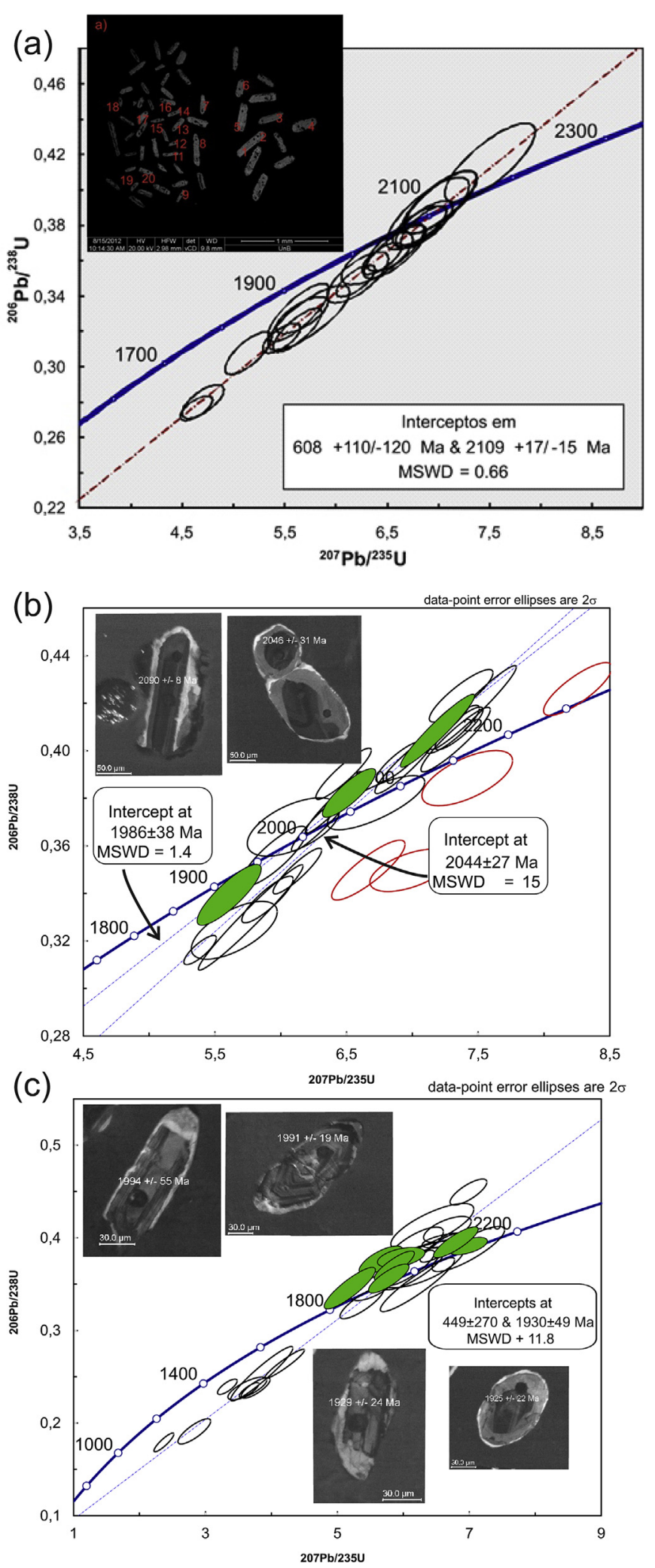

Fig. 5. $\mathrm{U}-\mathrm{Pb}$ concordia diagrams and representative images of the analyzed zircon grains. (a) GL-479 (São Joãozinho orthogneiss). (b) SU-541 (Vertentes Complex). Inset shows catholuminescence images of a euedral zircon with oscillatory zoning (left) and a core of oscillatory zoned zircon with an embayed morphology (right). (c) SU-515 (felsic gneiss). Green elipses in (b) and (c) are analyses from zircon crystals with Th/ $\mathrm{U}<0.1$; red elipses are analyses not used in age calculations. (For interpretation of the references to colour in this figure legend, the reader is referred to the web version of this article.)

\section{3. $\mathrm{U}-\mathrm{Pb}$ results}

\subsubsection{Migmatitic orthogneisses}

3.3.1.1. Sample SU-497 (Salgadinho Complex). Nineteen analyses were performed on 19 grains. The grains are subhedral to euhedral, show oscillatory zoning and have overgrowth rims of variable width (Fig. 4a). The analyses yielded a discordia with upper intercept at $2057 \pm 20 \mathrm{Ma}(\mathrm{MSWD}=2.4)$, which is taken as our best estimate for emplacement of the protolith. This age is similar within error to the ${ }^{207} \mathrm{~Pb} /{ }^{206} \mathrm{~Pb}$ weighted mean age of $2065 \pm 13 \mathrm{Ma}$ obtained in a cluster of ten concordant analyses (Fig. 4a).

3.3.1.2. Sample GL-476 (Cabaceiras unit). Twenty one analyses were obtained from 21 zircon grains. The grains have rounded morphologies and most present internal fractures (Fig. 4b). Regression of all data gave a discordia with upper intercept at $2055 \pm 23$ Ma (MSWD = 2.3; Fig. 4b), which is considered to represent the crystallization age of the protolith. This age is similar to that found in sample SU-497. A core spot (\#z09) with Th/U ratio lower than 0.1 yielded a ${ }^{207} \mathrm{~Pb} /{ }^{206} \mathrm{~Pb}$ value of $2075 \pm 14 \mathrm{Ma}$. It is possible that this grain is a xenocryst formed in a previous metamorphic event.

3.3.1.3. Sample RS-234. Twenty six analyses were performed on 26 six grains. They define a discordia line with upper intercept at $2183 \pm 9 \mathrm{Ma}$ and lower intercept at $604 \pm 20 \mathrm{Ma}(\mathrm{MSWD}=0.55$; Fig. 4c). Twelve analyses that plot close to concordia yielded a ${ }^{207} \mathrm{~Pb} /{ }^{206} \mathrm{~Pb}$ weighted average age of $2170 \pm 70 \mathrm{Ma}$ (MSWD $=0.007$ ), which is in accordance with the upper intercept age. These results indicate that the crystallization age of this orthogneiss is considerably older than that of samples SU-497 and GL-476. Despite the field and petrographic resemblance of this sample to other migmatitic orthogneisses, it differs on geochemical grounds (see Section 4). The lower intercept age corresponds to metamorphism related to the Brasiliano-Pan African event and is constrained by analysis Z08 (Table 1), which yielded a ${ }^{206} \mathrm{~Pb} /{ }^{238} \mathrm{U}$ age of $612 \pm 12 \mathrm{Ma}$ and has $\mathrm{Th} / \mathrm{U}$ ratio less than detection limit (0.00).

\subsubsection{Orthoamphibolite (RS-423)}

Eleven analyses were accomplished on 11 zircon crystals of sample RS-423. Two groups of zircons with different discordia ages were identified (Fig. 4d). The first group is formed by larger zircons with oscillatory zoning. Six analyses (Z04, Z06, Z07, Z08, Z09, Z10) that plot close to concordia yielded a discordia with upper intercept at $2042 \pm 11 \mathrm{Ma}$. This age is interpreted as the crystallization age of the protolith in view of the elevated $\mathrm{Th} / \mathrm{U}$ ratios $(>0.3)$ coupled with low MSWD (0.68). The other group is characterized by smaller elongated zircons with faces well preserved. Five discordant analyses (Z11, Z14, Z16, Z18, Z19) define a discordia with upper intercept at $1996 \pm 13 \mathrm{Ma}$. Th/U ratios lower than 0.1 obtained from four analyses of this group (\#Z11,\#Z16, \#Z18, \#Z19) suggest a metamorphic origin.

\subsubsection{São Joaozinho augen gneiss (GL-479)}

Zircon grains from sample GL-479 are yellowish to colourless, and present well preserved prismatic habit and faces (Fig. 5a). Twenty one analyses were obtained from 20 grains. The weighted mean ${ }^{207} \mathrm{~Pb} /{ }^{206} \mathrm{~Pb}$ age of $2100 \pm 9.7 \mathrm{Ma}$ obtained in a cluster of nine concordant analyses (less than $5 \%$ discordance) and with $\mathrm{Th} / \mathrm{U}$ ratios greater than 0.1 is taken as the best estimate for the age of crystallization of the protolith. This age is similar to the upper intercept age of $2109 \pm 15$ Ma (MSWD $=0.66$ ) obtained by regression of all data. 
Table 2

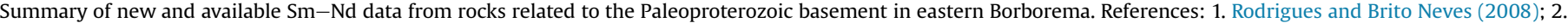
Brito Neves et al. (2001b); 3. Brito Neves et al. (2001a); 4. Dantas, 1997; 5. Van Schmus et al. (2011); 6. This paper.

\begin{tabular}{|c|c|c|c|c|c|c|c|c|c|c|}
\hline Sample & S lat & W long & $\mathrm{Nd}(\mathrm{ppm})$ & $\mathrm{Sm}(\mathrm{ppm})$ & ${ }^{147} \mathrm{Sm} /{ }^{144} \mathrm{Nd}$ & ${ }^{143} \mathrm{Nd} /{ }^{144} \mathrm{Nd}$ & eNd (now) & eNd (2050) & $\mathrm{T}_{\mathrm{DM}}(\mathrm{Ga})$ & \\
\hline \multicolumn{11}{|c|}{ Cabaceiras Complex } \\
\hline GL-157 & 7.464 & 36.342 & 7.601 & 1.921 & 0.1528 & 0.511751 & -17.31 & -5.82 & 3.28 & 6 \\
\hline GL-217 & 7.484 & 36.284 & 38.113 & 7.9453 & 0.126 & 0.51129 & -26.29 & -7.81 & 3.06 & 6 \\
\hline GL-476 & 7.483 & 36.303 & 16.343 & 2.304 & 0.0852 & 0.510729 & -37.25 & -8.08 & 2.74 & 6 \\
\hline GL-487 & 7.179 & 36.050 & 49.883 & 9.198 & 0.1115 & 0.510895 & -33.99 & -11.72 & 3.22 & 6 \\
\hline RS-046 & 7.594 & 36.335 & 31.581 & 7.78 & 0.0915 & 0.510706 & -37.68 & -10.18 & 2.92 & 6 \\
\hline RS-07B & 7.524 & 36.336 & 1.911 & 0.906 & 0.2865 & 0.512598 & -0.78 & -24.26 & & 6 \\
\hline CG-744 & 7.242 & 35.517 & 34.408 & 5.618 & 0.09874 & 0.51113 & -29.34 & -3.80 & 2.55 & \\
\hline CG-394 & 7.446 & 35.753 & 15.267 & 4.255 & 0.16854 & 0.51214 & -9.64 & -2.35 & 3.12 & \\
\hline SCB-Mi-ITB & 7.392 & 35.658 & 18.93 & 3.11 & 0.09976 & 0.51096 & -32.73 & -7.38 & 2.81 & \\
\hline SPP-MI-BJL & 7.463 & 35.828 & & & 0.1171 & 0.51102 & -31.56 & -10.75 & 3.25 & \\
\hline SCB-Gn-K44 & 7.215 & 35.523 & 18.46 & 3.8 & 0.12436 & 0.51141 & -23.9 & -5.04 & 2.81 & 4 \\
\hline \multicolumn{11}{|c|}{ Salgadinho Complex } \\
\hline RS-155 & 7.472 & 36.160 & 25.05 & 3.195 & 0.0771 & 0.51066 & -38.59 & -7.31 & 2.65 & 6 \\
\hline RS-313 & 7.573 & 36.205 & 281.565 & 40.904 & 0.0878 & 0.510872 & -34.45 & -5.97 & 2.62 & 6 \\
\hline \multicolumn{11}{|c|}{ São Joãozinho Orthogneiss } \\
\hline GL-479 & 7.284 & 36.275 & 55.921 & 10.514 & 0.1136 & 0.511059 & -30.81 & -9.07 & 3.03 & 6 \\
\hline \multicolumn{11}{|c|}{ Vertentes/Floresta Complex } \\
\hline $04-004 \mathrm{u}$ & 7.895 & 35.96 & 25.07 & 4.46 & 0.1076 & 0.511361 & -24.9 & -1.61 & 2.43 & \\
\hline CG-360 & 7.423 & 35.819 & 18.228 & 3.103 & 0.10294 & 0.51118 & -28.4 & -3.92 & 2.58 & \\
\hline CG-389 & 7.416 & 35.754 & 2.412 & 0.421 & 0.10565 & 0.51145 & -23.2 & 0.63 & 2.26 & \\
\hline CG-426 & 7.300 & 35.513 & 19.787 & 9.772 & 0.29862 & 0.51204 & -11.64 & -38.32 & & \\
\hline CG-427 & 7.300 & 35.496 & 21.273 & 4.717 & 0.13408 & 0.51168 & -18.67 & -2.31 & 2.63 & \\
\hline $94-115$ & 7.67 & 36.081 & 17.61 & 3.01 & 0.10352 & 0.511441 & -23.4 & 1.02 & 2.22 & \\
\hline SPP-Gn-13 & 7.469 & 35.928 & 29.326 & 6.035 & 0.1247 & 0.51162 & -19.96 & -1.03 & 2.46 & \\
\hline SPP-Th-NH & 7.450 & 35.910 & 6.692 & 1.194 & 0.1079 & 0.51141 & -24.05 & -0.73 & 2.37 & \\
\hline SPP-Gn-SQ & 7.450 & 35.910 & 4.91 & 0.86 & 0.10649 & 0.51155 & -21.28 & 2.37 & 2.13 & \\
\hline SCB-Gn-GAV & 7.425 & 35.958 & 9.68 & 1.88 & 0.11761 & 0.51161 & -20.15 & 0.63 & 2.29 & \\
\hline SCB-Ag-ITA2 & 7.383 & 35.750 & 89.415 & 14.74 & 0.0996 & 0.51121 & -27.84 & -2.47 & 2.46 & 4 \\
\hline SPP-Gn-PDS & 7.462 & 35.868 & 27.8 & 3.97 & 0.08644 & 0.51087 & -34.41 & -5.66 & 2.61 & 4 \\
\hline CA-42 & 8.028 & 35.667 & 14.834 & 2.980 & 0.1215 & 0.511541 & -21.4 & -1.74 & 2.5 & 6 \\
\hline
\end{tabular}

\subsubsection{Banded gneiss of Vertentes Complex (SU-541)}

Twenty six analyses were conducted of 26 zircon crystals from sample SU-541. Five analyses have discordant ages greater than $10 \%$ and are not considered in the following discussion. From the twenty one concordant or slightly discordant ages, eighteen are from grains with $\mathrm{Th} / \mathrm{U}$ ratios greater than 0.1 , among which three distinct age groups may be recognized (Fig. 5b). The first group comprises three analyses with ${ }^{207} \mathrm{~Pb} /{ }^{206} \mathrm{~Pb}$ ages between $2.16 \mathrm{Ga}$ and $2.23 \mathrm{Ga}$ that cannot be clustered to give a mean age. It is possible that these analyses are from xenocrystic grains, in which case their ${ }^{207} \mathrm{~Pb} /{ }^{206} \mathrm{~Pb}$ ages represent minimum estimates of their crystallization ages.

The second age group is formed by a cluster of five analyses (\#006-Z04, \#035-Z22, \#040-Z25, \#41-Z26, \#042-Z27) that have ${ }^{207} \mathrm{~Pb} /{ }^{206} \mathrm{~Pb}$ ages varying from 2.08 to $2.12 \mathrm{Ga}$ and gave a weighted average mean age of $2096 \pm 23 \mathrm{Ma}$. The dated grains are characterized by well-developed concentric oscillatory zoning (Fig. 5b) and, therefore, the age of $2096 \mathrm{Ma}$ is interpreted as the age of crystallization of the protolith.

The third group is formed by ten analyses that align along a discordia line and have an upper intercept age of $2044 \pm 27 \mathrm{Ma}$ (Fig. 5b). In spite of the high MSWD (=15), this age is considered representative since the most concordant analyses (five grains) gave a weighted average of $2060 \pm 14 \mathrm{Ma}$, which overlap within error with the discordia age. The age of 2044 Ma could be interpreted either as the minimum crystallization age of the protolith or as resulting from metamorphic disturbance. The latter interpretation is favored in view of: (a) the age of $2048 \pm 22 \mathrm{Ma}$ obtained in a grain with low Th/U ratio (0.08; \#039-Z24) and, therefore, of presumed metamorphic origin; (b) the grains show faint oscillatory zoning and embayments cutting the concentric zoning (Fig. 5b), suggesting, respectively, homogenization and dissolution during metamorphism.
Analysis 039-Z24 and two grains with low Th/U (\#023-Z15 and \#029-Z18) define another discordia with an upper intercept of $1986 \pm 38 \mathrm{Ma}$. The significance of this age is uncertain but it may correspond to another metamorphic event (see Discussion).

\subsubsection{Felsic gneiss (SU-515)}

Twenty nine analyses were conducted on 29 zircons from sample SU-515. Most analyses are discordant. Six analyses from grains with $\mathrm{Th} / \mathrm{U}$ ratio $>0.18$ are less than $5 \%$ discordant. One of these (analysis \#032-Z23) yielded a ${ }^{207} \mathrm{~Pb} /{ }^{206} \mathrm{~Pb}$ age of $2106 \pm 66 \mathrm{Ma}$, and possibly was obtained in a xenocrystic grain present in the zircon population. The other five analyses (\#029Z20, \#026-Z17, \#005-Z03, \#016-Z10, 027-Z18) can be clustered to give a weighted mean ${ }^{207} \mathrm{~Pb} /{ }^{206} \mathrm{~Pb}$ age of $1981 \pm 23 \mathrm{Ma}$ (Fig. $5 \mathrm{c}$ ). This age is the preferred estimate for the crystallization of the protolith because the analyzed zircons are euhedral and show well-

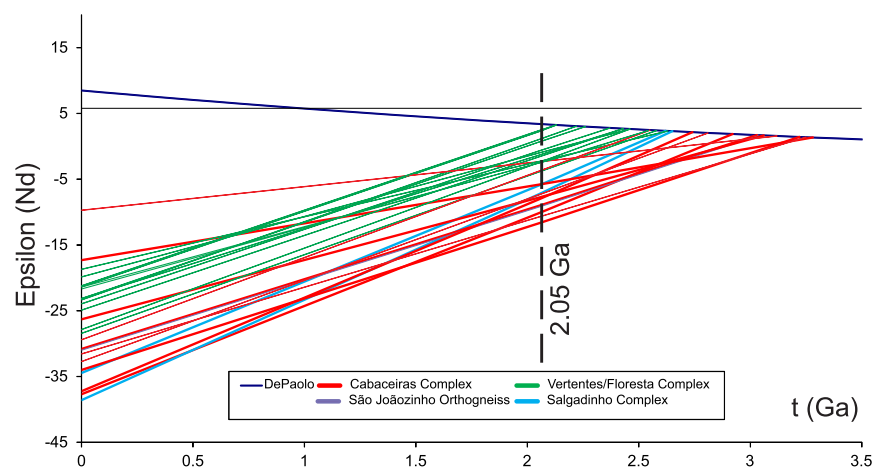

Fig. 6. Nd evolution paths versus age for Paleoproterozoic basement rocks from eastern Borborema. 
Table 3

\begin{tabular}{|c|c|c|c|c|c|c|c|c|c|c|c|c|c|c|c|c|c|c|c|c|c|c|}
\hline Sample & GL-215 & GL-18B & GL-024 & GL-476 & RS-46 & RS-396A & RS-408 & GL-485 & GL-487 & RS-424 & RS-234 & CIV-30B* & SU-497 & SU-503 & RS-155 & RS-215 & RS-218 & RS-323 & RS-165 & RS-373 & RS-250 & CIV-61* \\
\hline Unity & Cabaceira & & & & & & & & & & & Salgadinho & & & & & & & & & & \\
\hline \multicolumn{23}{|c|}{ Major elements (wt.\%) } \\
\hline $\mathrm{SiO}_{2}$ & 67.45 & 72.37 & 70.22 & 71.35 & 72.91 & 71.63 & 65.67 & 67.05 & 70.48 & 72.82 & 75.79 & 71.68 & 74.63 & 70.91 & 68.27 & 73.14 & 68.78 & 70.27 & 66.11 & 65.22 & 47.52 & 71.09 \\
\hline $\mathrm{Al}_{2} \mathrm{O}_{3}$ & 15.3 & 15.09 & 15.64 & 15.37 & 14.17 & 15.51 & 16.24 & 16.29 & 12.78 & 12.17 & 11.84 & 14.32 & 13.02 & 14.73 & 16.28 & 13.81 & 15 & 14.12 & 16.76 & 15.91 & 16.96 & 15.55 \\
\hline $\mathrm{Fe}_{2} \mathrm{O}_{3}$ & 2.8 & 1.14 & 1.77 & 1.66 & 1.67 & 1.97 & 3.93 & 2.69 & 4.82 & 4.15 & 2.68 & 3.42 & 1.59 & 3.94 & 3.43 & 2.97 & 3.8 & 4.01 & 4.09 & 4.94 & 12.17 & 0.79 \\
\hline MnO & 0.04 & 0.02 & 0.02 & 0.02 & 0.02 & 0.02 & 0.05 & 0.03 & 0.06 & 0.04 & 0.07 & 0.09 & 0.04 & 0.07 & 0.06 & 0.07 & 0.07 & 0.08 & 0.08 & 0.09 & 0.16 & 0.02 \\
\hline $\mathrm{MgO}$ & 1.67 & 0.24 & 0.62 & 0.36 & 0.56 & 0.48 & 1.61 & 1.07 & 0.47 & 0.67 & 0.21 & 0.73 & 0.35 & 0.84 & 0.94 & 0.86 & 1.61 & 1.37 & 1.25 & 1.97 & 5.31 & 0.27 \\
\hline $\mathrm{CaO}$ & 2.15 & 1.2 & 1.89 & 1.77 & 1.42 & 2.23 & 3.49 & 2.16 & 1.92 & 1.29 & 1.02 & 2.61 & 1.23 & 3.08 & 3.87 & 2.56 & 3.44 & 3.57 & 4.36 & 3.96 & 8.06 & 1.4 \\
\hline $\mathrm{Na}_{2} \mathrm{O}$ & 3.85 & 4.19 & 4.3 & 3.76 & 3.44 & 4.75 & 4.5 & 4.52 & 2.51 & 2.28 & 2.72 & 4.02 & 3.22 & 4.43 & 4.24 & 3.68 & 3.78 & 3.34 & 4.35 & 3.86 & 2.04 & 3.99 \\
\hline $\mathrm{K}_{2} \mathrm{O}$ & 5.55 & 5.05 & 4.43 & 4.88 & 5.08 & 2.7 & 2.9 & 4.72 & 5.3 & 5.41 & 4.68 & 2.1 & 4.82 & 1.11 & 1.8 & 2.29 & 2.43 & 2.21 & 1.81 & 2.59 & 4.26 & 5.31 \\
\hline $\mathrm{TiO}_{2}$ & 0.48 & 0.18 & 0.32 & 0.24 & 0.2 & 0.23 & 0.46 & 0.44 & 0.64 & 0.56 & 0.22 & 0.27 & 0.25 & 0.32 & 0.32 & 0.27 & 0.35 & 0.33 & 0.35 & 0.4 & 1.13 & 0.11 \\
\hline${ }_{2} \mathrm{O}_{5}$ & 0.27 & 0.06 & 0.12 & 0.05 & 0.09 & 0.08 & 0.16 & 0.18 & 0.16 & 0.11 & 0.04 & 0.113 & 0.06 & 0.08 & 0.14 & 0.08 & 0.1 & 0.11 & 0.14 & 0.12 & 0.86 & 0.049 \\
\hline LOI & 0.1 & 0.3 & 0.4 & 0.4 & 0.1 & 0.3 & 0.4 & 0.5 & 0.6 & 0.5 & 0.4 & 0.5 & 0.5 & 0.3 & 0.5 & 0.1 & 0.4 & 0.4 & 0.5 & 0.7 & 0.9 & 0.7 \\
\hline Total & 99.47 & 99.84 & 99.73 & 99.85 & 99.85 & 99.89 & 99.71 & 99.67 & 99.73 & 99.81 & 99.67 & 99.85 & 99.7 & 99.81 & 99.81 & 99.82 & 99.76 & 99.82 & 99.79 & 99.76 & 99.37 & 99.26 \\
\hline \multicolumn{23}{|c|}{ Trace elements (ppm) } \\
\hline $\mathrm{Ni}$ & 26.4 & 1.3 & 5.9 & 2 & & & & 12 & 4.4 & & 0.9 & $<20$ & $<20$ & 4.9 & 2 & 1.3 & 14.8 & 1.9 & 6.7 & 5.8 & 19.1 & $<20$ \\
\hline Co & & & & & 3.3 & 2.6 & 7.9 & & & 5.6 & 1.1 & 70.9 & 33.1 & 31.3 & 4.5 & 4.3 & 9.2 & 7.5 & 7.4 & 10.5 & 28.3 & 51.9 \\
\hline Sc & 7.6 & 1.5 & 4.1 & 2.7 & 3 & 2 & 7 & 6.7 & 6.1 & 8 & 4 & 7 & 3 & 11 & 5 & 7 & 8 & 11 & 8 & 11 & 25 & 2 \\
\hline V & 35 & $<8$ & 18 & 18 & & & & 28 & 24 & & $<8$ & 21 & 9 & 25 & 18 & 34 & 53 & 70 & 40 & 74 & 217 & $<8$ \\
\hline $\mathrm{Cu}$ & 20.8 & 1.7 & 7 & 4.3 & 12.9 & 8.9 & 5.7 & 10.8 & 3.3 & 3.8 & 11.9 & 4.9 & 1 & 3.8 & 2.8 & 3.9 & 2.6 & 4.6 & 12.1 & 5.2 & 107 & 27.6 \\
\hline $\mathrm{Pb}$ & 18.4 & 7.5 & 3.9 & 6.2 & 2.3 & 2.1 & 4.8 & 5.2 & 3.6 & 4 & 6.1 & 0.7 & 6.5 & 0.6 & 3.1 & 1.6 & 2.9 & 1.4 & 1.6 & 2.4 & 6 & 28.7 \\
\hline $\mathrm{n}$ & 44 & 37 & 35 & 35 & 29 & 41 & 78 & 52 & 60 & 41 & 40 & 51 & 23 & 34 & 51 & 43 & 51 & 38 & 65 & 57 & 116 & 13 \\
\hline 1o & 0.7 & $<0.1$ & $<0.1$ & $<0.1$ & 2.6 & $<0.1$ & 0.2 & $<0.1$ & 0.5 & 3.3 & 0.2 & 0.5 & 0.4 & 0.8 & 0.1 & 3.8 & 0.1 & 2.9 & 0.8 & 2.3 & 0.7 & 0.1 \\
\hline b & 193 & 148.3 & 115.4 & 123.8 & 101.9 & 106.4 & 76.2 & 182.8 & 91.9 & 220.9 & 78.1 & 57.1 & 124 & 25.8 & 72.2 & 60.5 & 73.5 & 71 & 47.7 & 76.4 & 156.4 & 92 \\
\hline Ва & 2504 & 804 & 1133 & 654 & 588 & 460 & 952 & 1384 & 1454 & 769 & 1731 & 903 & 1969 & 733 & 602 & 924 & 947 & 900 & 643 & 879 & 2689 & 4820 \\
\hline $\mathrm{Sr}$ & 1422.3 & 282.3 & 548.3 & 280.2 & 200.6 & 290.2 & 529 & 656.6 & 144.9 & 72.3 & 127.8 & 284.4 & 241.1 & 450.8 & 686.8 & 256 & 417.1 & 274.4 & 580.2 & 320.5 & 400.3 & 1578 \\
\hline a & 20.6 & 24.9 & 20.5 & 23.2 & 13.8 & 20.6 & 20.8 & 24.4 & 19.4 & 17.5 & 13.8 & 15.1 & 13.1 & 16.9 & 18.3 & 12.9 & 17.1 & 13.5 & 18.2 & 17.4 & 24.5 & 21.5 \\
\hline $\mathrm{Ta}$ & 1.1 & 0. & 0.2 & 0.2 & 0.1 & 0.4 & 0.3 & 0.4 & 0.5 & 1.3 & 1.3 & 0.4 & 0.8 & 0.2 & 0.2 & 0.4 & 0.6 & 0.4 & 0.2 & 0.6 & 1.2 & 0.2 \\
\hline b & 13.4 & 3 & 4 & 3 & 3.2 & 4.9 & 6. & 8.1 & 14.7 & 27.1 & 14.7 & 4.1 & 9.7 & 3.7 & 10.4 & 7.7 & 9.1 & 7.8 & 4.5 & 11.6 & 17 & 6.3 \\
\hline $\mathrm{Hf}$ & 7.7 & 4.5 & 6.3 & 4.7 & 4.1 & 3.4 & 4.1 & 7 & 14.6 & 15.6 & 11.1 & 3 & 5.3 & 4.7 & 4.1 & 3.6 & 4.7 & 3.3 & 2.3 & 4.7 & 3.6 & 4.8 \\
\hline $\mathrm{Zr}$ & 262.7 & 148.8 & 236.9 & 147.6 & 124.5 & 132.7 & 158.7 & 274.6 & 585.4 & 574.5 & 375.2 & 95.6 & 171.7 & 167.1 & 147.1 & 127.4 & 157.3 & 109.1 & 89.2 & 174.2 & 135.1 & 115.8 \\
\hline $\mathrm{y}$ & 11 & 2.7 & 3 & 3.2 & 8.9 & 3.3 & 4.9 & 5.6 & 32.6 & 72.7 & 92.6 & 13.7 & 17.4 & 12.4 & 6.3 & 14.9 & 14.4 & 15 & 11.3 & 22 & 37.9 & 4.6 \\
\hline Th & 28.3 & 20.1 & 17.9 & 19.5 & 13.5 & 8 & 1. & 21.9 & 51 & 43.3 & 12.1 & 2 & 12 & 3.5 & 11.2 & 7.4 & 9 & 6.2 & 35 & 7.5 & 11.6 & 14.8 \\
\hline 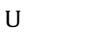 & 5.5 & 4.1 & 1 & 0.8 & 0.4 & 1. & 0.6 & 1.7 & 0.8 & 3 & 3.7 & 0. & 2 & 0.4 & 0.7 & 0.8 & 2.1 & 1 & 0.7 & 1.2 & 3.8 & 2.1 \\
\hline La & 84.7 & 42.2 & 44 & 44.3 & 41 & 22.8 & 26.9 & 594 & 56.4 & 98.6 & 67.3 & 25. & 42.5 & 25 & 45.4 & 30.1 & 25.1 & 23.6 & 20.4 & 34.1 & 81.9 & 24.5 \\
\hline $\mathrm{Ce}$ & 158.2 & 64.5 & 74.9 & 75.9 & 76.6 & 39 & 47 & 103.1 & 105.7 & 197.4 & 146.6 & 30.5 & 84.3 & 53.8 & 81.3 & 60.1 & 46.1 & 45.7 & 39.9 & 64.3 & 167.8 & 45 \\
\hline $\operatorname{Pr}$ & 17.32 & 7.08 & 7.33 & 7.66 & 8.71 & 4.23 & 5.28 & 10.43 & 12.27 & 22.15 & 18.1 & 4.66 & 7.95 & 6.32 & 8.87 & 6.39 & 5.48 & 4.88 & 4.82 & 7.39 & 20.89 & 5.01 \\
\hline $\mathrm{Nd}$ & 57.6 & 23.4 & 24 & 24.8 & 29.7 & 13.1 & 17.7 & 35.2 & 49.6 & 79 & 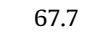 & 16 & 26.5 & 26.2 & 28.9 & 21.5 & 18.7 & 16.9 & 17.9 & 25.3 & 81.7 & 17.9 \\
\hline $\mathrm{Sm}$ & 8.13 & 3.19 & 3.18 & 3.25 & 4.81 & 2.21 & 2.87 & 4.36 & 8.55 & 14.56 & 13.55 & 2.7 & 4.27 & 4.55 & 3.65 & 3.58 & 3.63 & 2.64 & 2.99 & 4.59 & 14.17 & 3.03 \\
\hline Eu & & & & & & & & & & 177 & 1.67 & 07 & & 1.1 & 1.22 & .71 & 0.79 & 0.65 & 0.87 & 1.01 & 3.01 & 0.91 \\
\hline Gd & 4.37 & 1.81 & 1.66 & 1.94 & 3.4 & 1.26 & 1.86 & 2 & 7.85 & 13.13 & 12.65 & 2.3 & 3.34 & 3.5 & 2.16 & 2.72 & 2.86 & 2.14 & 2.26 & 4 & 10.3 & 1.98 \\
\hline $\mathrm{Tb}$ & & & & 0.21 & & & 0.26 & & & 2.25 & 2.37 & & 0.52 & 0.5 & 0.28 & 0.46 & 0.47 & 0.37 & 0.35 & 0.64 & 1.45 & 0.25 \\
\hline Dy & & & & 0.79 & 1. & 0 & & & 6 & 13.08 & 14.07 & 2. & 2.97 & 2.61 & 1.2 & 2.33 & 2.34 & 2.09 & 1.88 & 3.64 & 7.19 & 1.04 \\
\hline Ho & 0.36 & 0. & 0 & 0 & 0. & 0. & 0.1 & & & 2.6 & 3.29 & 0. & 0.6 & 0.45 & 0.22 & 0.51 & 0.51 & 0.46 & 0.38 & 0.81 & 1.25 & 0.15 \\
\hline $\mathrm{Er}$ & 0. & 0. & & 0.23 & 0.77 & 0.2 & 0.4 & 0.51 & 3.84 & 7 & 10.23 & 1. & 1.87 & 1.04 & 0.54 & 1.53 & 1.53 & 1.45 & 1.08 & 2.35 & 3.44 & 0.32 \\
\hline $\mathrm{Tm}$ & 0.14 & 0.03 & 0.03 & 0.03 & 0.13 & 0.04 & 0.07 & 0.07 & 0.56 & 1.12 & 1.67 & 0.1 & 0.29 & 0.14 & 0.08 & 0.24 & 0.22 & 0.24 & 0.18 & 0.35 & 0.51 & 0.03 \\
\hline $\mathrm{Yb}$ & & & & 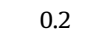 & 0. & & 0. & 0.39 & 3.26 & 6.62 & 11.61 & 1. & & 0.8 & 0.53 & 1.62 & 1.5 & 1.72 & 1.02 & 2.56 & 3.24 & 0.29 \\
\hline Lu & 0.11 & 0.02 & 0.03 & 0.03 & 0.1 & 0.04 & 0.07 & 0.06 & 0.46 & 0.84 & 1.73 & 0.17 & 0.31 & 0.13 & 0.09 & 0.24 & 0.23 & 0.28 & 0.16 & 0.38 & 0.48 & 0.04 \\
\hline$\sum \mathrm{REE}$ & 337.15 & 144.07 & 157.47 & 160.23 & 169.33 & 84.76 & 105.02 & 219.36 & 260.47 & 460.52 & 372.54 & 87.72 & 178.48 & 126.23 & 174.44 & 132.03 & 109.46 & 103.12 & 94.19 & 151.42 & 397.33 & 100.45 \\
\hline Sample & \multicolumn{2}{|c|}{ GL-093A } & \multicolumn{2}{|c|}{ GL-108 } & \multicolumn{2}{|c|}{ GL-152 } & GL-339 & \multicolumn{2}{|c|}{ GL-446 } & \multicolumn{2}{|c|}{ GL-447 } & GL-479 & \multicolumn{2}{|c|}{ GL-480 } & \multicolumn{2}{|c|}{ GL-481 } & \multicolumn{2}{|c|}{ GL-093A } & GL-1 & 108 & & L-152 \\
\hline Unity & & o Joaozin & & & & & & & & & & & & & & & & & & & & \\
\hline & & NL. & & & & & & & & & & & & & & & & & & & & \\
\hline $\mathrm{O}_{2}$ & & 65.53 & & 2.47 & . & & 62.07 & & 57.03 & . & & 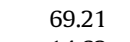 & & 68.5 & & .76 & & & & 1.32074 & & 60.95252 \\
\hline $\mathrm{Al}_{2} \mathrm{O}_{3}$ & & 16.07 & & 3.52 & 13.3 & & 17.72 & & 20.65 & & .07 & 14.62 & & 15 & & 1.19 & $15.5 \mathrm{~s}$ & 921 & & 5.57502 & & 15.55795 \\
\hline $\mathrm{Fe}_{2} \mathrm{O}_{3}$ & & 5.13 & & 3.87 & 5.3 & & 5.37 & & 5.11 & & 86 & 4.24 & & 4.29 & & .01 & & 01429 & & 5.755857 & & 5.810286 \\
\hline
\end{tabular}




\begin{tabular}{|c|c|c|c|c|c|c|c|c|c|c|c|c|c|c|c|c|}
\hline $\mathrm{MnO}$ & 0.08 & & .05 & 0.08 & 0.08 & 0.07 & 0.09 & & 0.06 & 0.06 & 0.09 & & 0.108381 & 0.111345 & & 0.11431 \\
\hline $\mathrm{MgO}$ & 0.3 & & .48 & 0.72 & 0.2 & 0.7 & 0.25 & & 0.37 & 0.4 & 0.21 & & 1.901905 & 2.021476 & & 2.141048 \\
\hline $\mathrm{CaO}$ & 2.85 & & .03 & 2.63 & 3.19 & 6.53 & 3.2 & & 2.57 & 2.2 & 3.78 & & 3.698667 & 3.749167 & & 3.799667 \\
\hline $\mathrm{Na}_{2} \mathrm{O}$ & 4.4 & & .74 & 3.29 & 5.11 & 6.03 & 4.4 & & 3.71 & 3.7 & 3.25 & & 3.406095 & 3.339024 & & 3.271952 \\
\hline $\mathrm{K}_{2} \mathrm{O}$ & 4.41 & & 13 & 3.82 & 4.8 & 2.7 & 4.83 & & 3.98 & 4.73 & 4.66 & & 5.195524 & 5.297631 & & 5.399738 \\
\hline $\mathrm{TiO}_{2}$ & 0.35 & & .37 & 0.6 & 0.46 & 0.5 & 0.39 & & 0.36 & 0.41 & 0.44 & & 0.707619 & 0.730405 & & 0.75319 \\
\hline $\mathrm{P}_{2} \mathrm{O}_{5}$ & 0.09 & & .11 & 0.22 & 0.14 & 0.2 & 0.11 & & 0.12 & 0.14 & $<0.01$ & & 0.148214 & 0.149762 & & 0.15131 \\
\hline LOI & 0.3 & & & 0.8 & 0.2 & 0 & 0 & & 0.4 & 0 & 0.1 & & 0.98 & 1.0425 & & 1.105 \\
\hline Total & 99.5 & & .76 & 99.65 & 99.33 & 99.51 & 99.4 & & 99.65 & 99.44 & 99.49 & & 99.82 & 99.76 & & 99.81 \\
\hline \multicolumn{17}{|c|}{ Trace elements (ppm) } \\
\hline $\mathrm{Ni}$ & 2.4 & & .8 & 3.1 & 0.8 & 2.1 & 1.3 & & 1.3 & 2.1 & 1.5 & \multicolumn{2}{|c|}{$<20$} & 15.3 & \multicolumn{2}{|r|}{5.7} \\
\hline Co & 2.9 & & 3 & 6.3 & 2.6 & 5.6 & 2.7 & & 4 & 4.1 & 2.9 & \multirow{2}{*}{\multicolumn{2}{|c|}{49.6}} & 12.6 & & 30.8 \\
\hline $\mathrm{Sc}$ & 9 & & & 10 & 12 & 8 & 11 & & 8 & 6 & 10 & \multirow{2}{*}{\multicolumn{2}{|c|}{$\begin{array}{l}12 \\
<8\end{array}$}} & 13 & & 15 \\
\hline V & $<8$ & & & 23 & $<8$ & 35 & 23 & & 13 & 16 & 33 & & & 55 & & 82 \\
\hline $\mathrm{Cu}$ & 7 & & .8 & 6.5 & 1.6 & 2.6 & 1.6 & & 5.4 & 3.7 & 2.5 & \multicolumn{2}{|r|}{55.2} & 25.7 & & 1.1 \\
\hline $\mathrm{Pb}$ & 4.7 & & .4 & 5.7 & 1.9 & 3.3 & 5.4 & & 5.5 & 6.6 & 2.1 & \multicolumn{2}{|r|}{2.8} & 5.1 & & 0.9 \\
\hline $\mathrm{Zn}$ & 86 & & & 91 & 57 & 14 & 102 & & 77 & 92 & 81 & \multicolumn{2}{|r|}{35} & 60 & & 71 \\
\hline Mo & 0.6 & & 6 & 1 & 0.4 & 0.2 & $<0.1$ & & 0.9 & 0.5 & 0.6 & \multicolumn{2}{|r|}{2.7} & 1.2 & & 0.5 \\
\hline $\mathrm{Rb}$ & 77.2 & & .9 & 98.2 & 61.2 & 40.3 & 68.4 & & 72.9 & 68 & 71.6 & \multirow{2}{*}{\multicolumn{2}{|c|}{$\begin{array}{c}22.4 \\
542\end{array}$}} & 69.5 & & 39 \\
\hline $\mathrm{Ba}$ & 3042 & 13 & & 1275 & 3731 & 1517 & 3202 & & 1890 & 2727 & 2900 & & & 1045 & & 582 \\
\hline $\mathrm{Sr}$ & 303.5 & & & 203.6 & 392.9 & 991.3 & 351.3 & & 251.5 & 268 & 386 & \multicolumn{2}{|c|}{386.8} & 471.3 & & 388.4 \\
\hline $\mathrm{Ga}$ & 25.6 & & .7 & 20.4 & 26.3 & 26.3 & 21.8 & & 25.4 & 26.5 & 28.6 & \multicolumn{2}{|r|}{17.7} & 20 & & 16.9 \\
\hline $\mathrm{Ta}$ & 0.5 & & .5 & 1.3 & 0.4 & 1.5 & 0.8 & & 0.6 & 0.6 & 0.5 & & 0.2 & 0.2 & & 0.4 \\
\hline $\mathrm{Nb}$ & 14.3 & & 8 & 19 & 10.7 & 33.7 & 18.4 & & 12.8 & 20 & 15.3 & & 5.3 & 7.1 & & 6.1 \\
\hline $\mathrm{Hf}$ & 13 & & 4 & 11.3 & 19.1 & 14.1 & 15 & & 10.4 & 14.5 & 19.4 & & 5.5 & 5.1 & & 3.7 \\
\hline $\mathrm{Zr}$ & 528.3 & & & 426.9 & 791.6 & 534.5 & 655.3 & & 399.2 & 556.4 & 806.2 & & 22.5 & 207 & & 110.4 \\
\hline $\mathrm{Y}$ & 38.8 & & .2 & 70.1 & 37.9 & 49.4 & 46.9 & & 52.9 & 67.1 & 37.4 & & 11.6 & 15.8 & & 14.7 \\
\hline Th & 8.4 & & .2 & 12.5 & 6.1 & 19.3 & 10.3 & & 7.6 & 9.2 & 9.2 & & 3.5 & 4.1 & & 5.2 \\
\hline U & 1.6 & & .1 & 2.5 & 2.2 & 4 & 1.7 & & 2.4 & 1.5 & 1.6 & & 0.9 & 0.4 & & 0.6 \\
\hline La & 94.1 & & 4 & 68.1 & 84.9 & 131.4 & 112.3 & & 69.4 & 111.8 & 132.1 & & 15.5 & 47.4 & & 16.1 \\
\hline $\mathrm{Ce}$ & 188.4 & & .3 & 142.4 & 158.9 & 251.7 & 223 & & 140.3 & 221.5 & 256.8 & & 35.5 & 88.5 & & 34.1 \\
\hline $\mathrm{Pr}$ & 22.12 & & .47 & 17.3 & 17.93 & 27.2 & 25.97 & & 16.35 & 25.84 & 27.83 & & 4.1 & 9.56 & & 3.93 \\
\hline $\mathrm{Nd}$ & 84.4 & & .8 & 69.4 & 63.5 & 88.7 & 94.8 & & 63.4 & 101.9 & 95.1 & & 16.3 & 35.7 & & 16.1 \\
\hline $\mathrm{Sm}$ & 13.01 & & .65 & 13.4 & 10.31 & 13.55 & 15.61 & & 10.74 & 16.6 & 14.11 & & 2.92 & 5.21 & & 3.19 \\
\hline Eu & 2.89 & & .39 & 1.73 & 4.56 & 2.7 & 3.48 & & 2.05 & 3.12 & 3.85 & & 1.13 & 1.16 & & 0.84 \\
\hline $\mathrm{Gd}$ & 9.66 & & .17 & 13.06 & 8.09 & 11.34 & 12.44 & & 10.02 & 15.11 & 10.52 & & 2.35 & 3.55 & & 2.81 \\
\hline $\mathrm{Tb}$ & 1.48 & & .33 & 2.17 & 1.26 & 1.64 & 1.69 & & 1.61 & 2.34 & 1.46 & & 0.35 & 0.51 & & 0.46 \\
\hline Dy & 8.06 & & .5 & 12.94 & 7.18 & 9.35 & 8.72 & & 9.59 & 13.44 & 7.35 & & 2 & 2.83 & & 2.47 \\
\hline Ho & 1.47 & & .53 & 2.61 & 1.38 & 1.86 & 1.8 & & 1.97 & 2.6 & 1.4 & & 0.46 & 0.54 & & 0.49 \\
\hline $\mathrm{Er}$ & 4.24 & & 36 & 7.53 & 4.01 & 5.04 & 4.71 & & 5.63 & 7.08 & 4.27 & & 1.48 & 1.5 & & 1.5 \\
\hline $\mathrm{Tm}$ & 0.63 & & .68 & 1.2 & 0.64 & 0.8 & 0.77 & & 0.83 & 1.06 & 0.6 & & 0.24 & 0.24 & & 0.21 \\
\hline $\mathrm{Yb}$ & 4.28 & & 62 & 7.49 & 4.02 & 4.81 & 4.51 & & 5.11 & 6.21 & 4.35 & & 1.81 & 1.6 & & 1.54 \\
\hline Lu & 0.67 & & 17 & 1.08 & 0.68 & 0.68 & 0.68 & & 0.72 & 0.93 & 0.72 & & 0.3 & 0.26 & & 0.23 \\
\hline$\sum \mathrm{REE}$ & 435.41 & & & 360.41 & 367.36 & 550.77 & 510.48 & & 337.72 & 529.53 & 560.46 & & 84.44 & 198.56 & & 83.97 \\
\hline Sample & VAN-03* & VAN-29* & VAN-58* & VAN-59* & VAN-52 ${ }^{a *}$ & VAN-53* & SU-551 & RS-128 & RS-190 & RS-326 & RS-328 & RS-426 & VAN-43* & VAN-45* & RS-145 & SU-515 \\
\hline Unity & Vertentes & & & & & & & & & & & & & & & \\
\hline Major $e$ & nents (wt.\%) & & & & & & & & & & & & & & & \\
\hline $\mathrm{SiO}_{2}$ & 66.42 & 58.44 & 64.15 & 66.04 & 57.7 & 63.12 & 57.19 & 58.16 & 53.87 & 58.55 & 61.32 & 63.86 & 69.78 & 69.95 & 70.3 & 73.72 \\
\hline $\mathrm{Al}_{2} \mathrm{O}_{3}$ & 15.48 & 16.49 & 14.65 & 15.61 & 15.03 & 16.51 & 20.19 & 17.02 & 18.19 & 16.5 & 16.82 & 16.69 & 14.85 & 15.47 & 15.01 & 13.45 \\
\hline $\mathrm{Fe}_{2} \mathrm{O}_{3}$ & 5.67 & 7.99 & 5.14 & 3.3 & 6.71 & 5 & 4.85 & 7.07 & 8.81 & 6.68 & 5.43 & 5.04 & 2.29 & 1.79 & 2.69 & 1.67 \\
\hline $\mathrm{MnO}$ & 0.1 & 0.1 & 0.11 & 0.07 & 0.13 & 0.1 & 0.07 & 0.11 & 0.13 & 0.1 & 0.1 & 0.09 & 0.07 & 0.05 & 0.05 & 0.06 \\
\hline $\mathrm{MgO}$ & 1.78 & 3.12 & 1.21 & 0.63 & 1.96 & 1.85 & 1.57 & 2.95 & 3.42 & 4.12 & 1.71 & 1.7 & 0.96 & 0.48 & 0.53 & 0.33 \\
\hline $\mathrm{CaO}$ & 2.54 & 4.23 & 2.66 & 1.96 & 4.6 & 4.45 & 5.55 & 5.89 & 6.25 & 5.83 & 3.68 & 3.86 & 1.36 & 1.44 & 2.43 & 1.76 \\
\hline $\mathrm{Na}_{2} \mathrm{O}$ & 3.45 & 2.97 & 3.74 & 4.39 & 2.74 & 4.22 & 4.32 & 4.04 & 4.4 & 3.31 & 4.76 & 3.88 & 4.45 & 4.74 & 3.75 & 3.49 \\
\hline $\mathrm{K}_{2} \mathrm{O}$ & 3.03 & 3.74 & 5.78 & 5.79 & 7.69 & 2.59 & 3.77 & 2.26 & 2.68 & 3.08 & 4.03 & 3.32 & 5.05 & 4.67 & 3.87 & 4.22 \\
\hline $\mathrm{TiO}_{2}$ & 0.55 & 0.84 & 0.65 & 0.41 & 1.03 & 0.52 & 0.65 & 0.75 & 0.98 & 0.58 & 0.44 & 0.61 & 0.2 & 0.22 & 0.26 & 0.19 \\
\hline $\mathrm{P}_{2} \mathrm{O}_{5}$ & 0.103 & 0.471 & 0.177 & 0.129 & 0.347 & 0.275 & 0.24 & 0.32 & 0.46 & 0.25 & 0.23 & 0.24 & 0.135 & 0.094 & 0.1 & 0.06 \\
\hline & & & & & & & & & & & & & & & ued on & t page) \\
\hline
\end{tabular}


Table 3 (continued)

\begin{tabular}{|c|c|c|c|c|c|c|c|c|c|c|c|c|c|c|c|c|}
\hline Sample & VAN-03* & VAN-29* & VAN-58* & VAN-59* & VAN-52 $2^{a *}$ & VAN-53* & SU-551 & RS-128 & RS-190 & RS-326 & RS-328 & RS-426 & VAN-43* & VAN-45* & RS-145 & SU-515 \\
\hline Unity & Vertentes & & & & & & & & & & & & & & & \\
\hline LOI & 0.7 & 1.3 & 0.7 & 0.6 & 1.1 & 1 & 1.1 & 1 & 0.4 & 0.6 & 0.7 & 0.4 & 0.2 & 0.4 & 0.8 & 0.9 \\
\hline Total & 99.84 & 99.67 & 98.99 & 98.94 & 99.04 & 99.64 & 99.51 & 99.6 & 99.62 & 99.61 & 99.19 & 99.67 & 99.36 & 99.29 & 99.75 & 99.85 \\
\hline \multicolumn{17}{|c|}{ Trace elements (ppm) } \\
\hline $\mathrm{Ni}$ & 45 & $<20$ & 32 & 45 & $<20$. & $<20$ & & 9.4 & 16.2 & 26.5 & 5.9 & 5.1 & 28 & 22 & 1.6 & $<20$ \\
\hline Co & 49.2 & 61.7 & 60.2 & 84.1 & 81.9 & 39.5 & 62.9 & 17.8 & 21.9 & 22.1 & 11.6 & 11.1 & 50.7 & 96.1 & 3.8 & 21.6 \\
\hline $\mathrm{Sc}$ & 13 & 15 & 11 & 6 & 15 & 9 & 11 & & & & & & 6 & 5 & & 3 \\
\hline $\mathrm{V}$ & 77 & 85 & 38 & 17 & 53 & 33 & 46 & 84 & 98 & 99 & 38 & 45 & 28 & 21 & 11 & 14 \\
\hline $\mathrm{Cu}$ & 26.6 & 32.7 & 10.9 & 1.1 & 31 & 13.7 & 10.2 & 22.3 & 24.9 & 5.4 & 10.3 & 4.7 & 2.9 & 1.5 & 10.8 & 2.7 \\
\hline $\mathrm{Pb}$ & 2.1 & 6.9 & 23.8 & 20.6 & 24.6 & 3 & 6.4 & 2.5 & 5.3 & 5.4 & 21.4 & 5.4 & 26.7 & 19.9 & 5.6 & 8 \\
\hline $\mathrm{Zn}$ & 83 & 95 & 61 & 27 & 27 & 61 & 62 & 66 & 79 & 42 & 77 & 67 & 33 & 54 & 40 & 40 \\
\hline Mo & 1 & 0.5 & 0.7 & 0.4 & 0.2 & 0.3 & 0.9 & 0.5 & 0.6 & 1.5 & 0.6 & 0.1 & 0.3 & 0.3 & 3.1 & 0.9 \\
\hline $\mathrm{Rb}$ & 145.9 & 90.1 & 98.1 & 85.2 & 145.4 & 72.9 & 89 & 45 & 86.8 & 77.6 & 70.9 & 141.6 & 110.8 & 72.7 & 93.4 & 112.2 \\
\hline $\mathrm{Ba}$ & 669 & 1658 & 5243 & 5457 & 5959 & 1576 & 2182 & 1348 & 1236 & 1499 & 4223 & 1648 & 3635 & 3597 & 1180 & 886 \\
\hline $\mathrm{Sr}$ & 308.1 & 603.7 & 2272 & 2759 & 970.3 & 844.8 & 946.1 & 906.5 & 879 & 666.4 & 1815.9 & 750.3 & 1183 & 1591 & 536.1 & 174.2 \\
\hline $\mathrm{Ga}$ & 20.8 & 17.8 & 18.9 & 19.1 & 14.1 & 19.9 & 23.3 & 18.4 & 22.2 & 17.2 & 20.3 & 20.2 & 14.2 & 19.1 & 17 & 19 \\
\hline $\mathrm{Ta}$ & 0.9 & 1 & 0.9 & 0.9 & 1.2 & 0.3 & 0.5 & 0.3 & 0.7 & 0.3 & 0.5 & 0.7 & $<0.1$ & 0.3 & 0.1 & 1 \\
\hline $\mathrm{Nb}$ & 10.7 & 13.8 & 14.5 & 13.5 & 17.4 & 5.1 & 10.9 & 6.8 & 12.2 & 8.4 & 11.3 & 13.2 & 5 & 7.8 & 5.1 & 11.4 \\
\hline $\mathrm{Hf}$ & 5.5 & 4.7 & 7.7 & 7.7 & 6.6 & 3.3 & 8.1 & 4 & 4.9 & 6 & 5.7 & 4.3 & 3.6 & 4.8 & 4 & 4.5 \\
\hline $\mathrm{Zr}$ & 177.5 & 164.6 & 313.8 & 293.7 & 253.5 & 136.6 & 316.1 & 152.8 & 195 & 220.9 & 241.1 & 154.9 & 117 & 139.5 & 136 & 149.6 \\
\hline $\mathrm{Y}$ & 21.2 & 24.9 & 29.8 & 21.2 & 24.4 & 10.8 & 16.8 & 15.5 & 23.7 & 14.3 & 16.2 & 11.8 & 15.8 & 7.6 & 7 & 11 \\
\hline Th & 16.4 & 24.7 & 8.7 & 10.4 & 6.7 & 5.8 & 8.8 & 1.1 & 5.4 & 1.6 & 11.1 & 2.6 & 5.9 & 6.4 & 7.5 & 11.8 \\
\hline $\mathrm{U}$ & 3 & 2.8 & 1.2 & 1 & 1.7 & 0.6 & 1.9 & 0.3 & 1.3 & 0.5 & 2.7 & 0.8 & 1.2 & 0.9 & 0.6 & 1.8 \\
\hline $\mathrm{La}$ & 44.3 & 74.7 & 108.2 & 93.5 & 54.2 & 49 & 44.5 & 29.7 & 45.3 & 33.2 & 72.7 & 49.8 & 30.7 & 24.6 & 30.7 & 33 \\
\hline $\mathrm{Ce}$ & 90 & 155.1 & 188.4 & 157.8 & 119.8 & 76.8 & 89.4 & 60.4 & 92.5 & 62.8 & 136 & 92.1 & 53.7 & 46.8 & 55.1 & 62.7 \\
\hline $\mathrm{Pr}$ & 10.69 & 16.47 & 23.51 & 19.02 & 16.22 & 9.02 & 9.73 & 7.62 & 11.56 & 7.59 & 15.77 & 10.17 & 5.98 & 5.26 & 5.85 & 6.44 \\
\hline $\mathrm{Nd}$ & 39.3 & 59.8 & 81.8 & 63.5 & 58.4 & 29.4 & 36.6 & 30 & 42.1 & 28 & 55.7 & 37.1 & 18.7 & 18.6 & 19.8 & 20.9 \\
\hline Sm & 7.51 & 9.83 & 13.52 & 10.86 & 9.63 & 4.49 & 5.91 & 5.13 & 7.97 & 5.13 & 8.45 & 5.23 & 3.19 & 3.02 & 2.57 & 3.33 \\
\hline $\mathrm{Eu}$ & 1.11 & 2.18 & 3.31 & 2.72 & 2.55 & 1.74 & 1.97 & 1.54 & 1.85 & 1.44 & 2.05 & 1.53 & 0.96 & 1.08 & 0.92 & 0.63 \\
\hline $\mathrm{Gd}$ & 6.06 & 6.58 & 7.66 & 5.72 & 5.72 & 2.64 & 4.65 & 3.91 & 5.73 & 3.7 & 5.26 & 3.63 & 2.32 & 1.78 & 1.62 & 2.26 \\
\hline $\mathrm{Tb}$ & 0.88 & 0.97 & 1.22 & 0.9 & 0.97 & 0.41 & 0.63 & 0.57 & 0.86 & 0.55 & 0.68 & 0.46 & 0.43 & 0.32 & 0.22 & 0.36 \\
\hline Dy & 4.2 & 4.72 & 5.56 & 3.88 & 4.48 & 1.91 & 3.21 & 2.97 & 4.44 & 2.74 & 3.09 & 2.08 & 2.28 & 1.65 & 1.28 & 2 \\
\hline Ho & 0.72 & 0.78 & 1.01 & 0.68 & 0.8 & 0.34 & 0.6 & 0.54 & 0.84 & 0.51 & 0.58 & 0.38 & 0.4 & 0.28 & 0.24 & 0.37 \\
\hline $\mathrm{Er}$ & 2.15 & 2.31 & 2.37 & 1.84 & 2.14 & 0.95 & 1.59 & 1.41 & 2.21 & 1.47 & 1.58 & 0.95 & 1.12 & 0.7 & 0.65 & 1.11 \\
\hline $\mathrm{Tm}$ & 0.36 & 0.34 & 0.39 & 0.24 & 0.27 & 0.15 & 0.24 & 0.22 & 0.33 & 0.21 & 0.23 & 0.16 & 0.16 & 0.1 & 0.11 & 0.17 \\
\hline $\mathrm{Yb}$ & 2.17 & 2.19 & 2.21 & 1.54 & 1.79 & 0.92 & 1.54 & 1.33 & 2.02 & 1.35 & 1.54 & 0.91 & 0.92 & 0.55 & 0.69 & 1.13 \\
\hline $\mathrm{Lu}$ & 0.34 & 0.32 & 0.36 & 0.26 & 0.26 & 0.14 & 0.21 & 0.2 & 0.3 & 0.2 & 0.23 & 0.14 & 0.14 & 0.08 & 0.12 & 0.18 \\
\hline$\sum$ REE & 209.79 & 336.29 & 439.52 & 362.46 & 277.23 & 177.91 & 200.78 & 145.54 & 218.01 & 148.89 & 303.86 & 204.64 & 121 & 104.82 & 119.87 & 134.58 \\
\hline
\end{tabular}



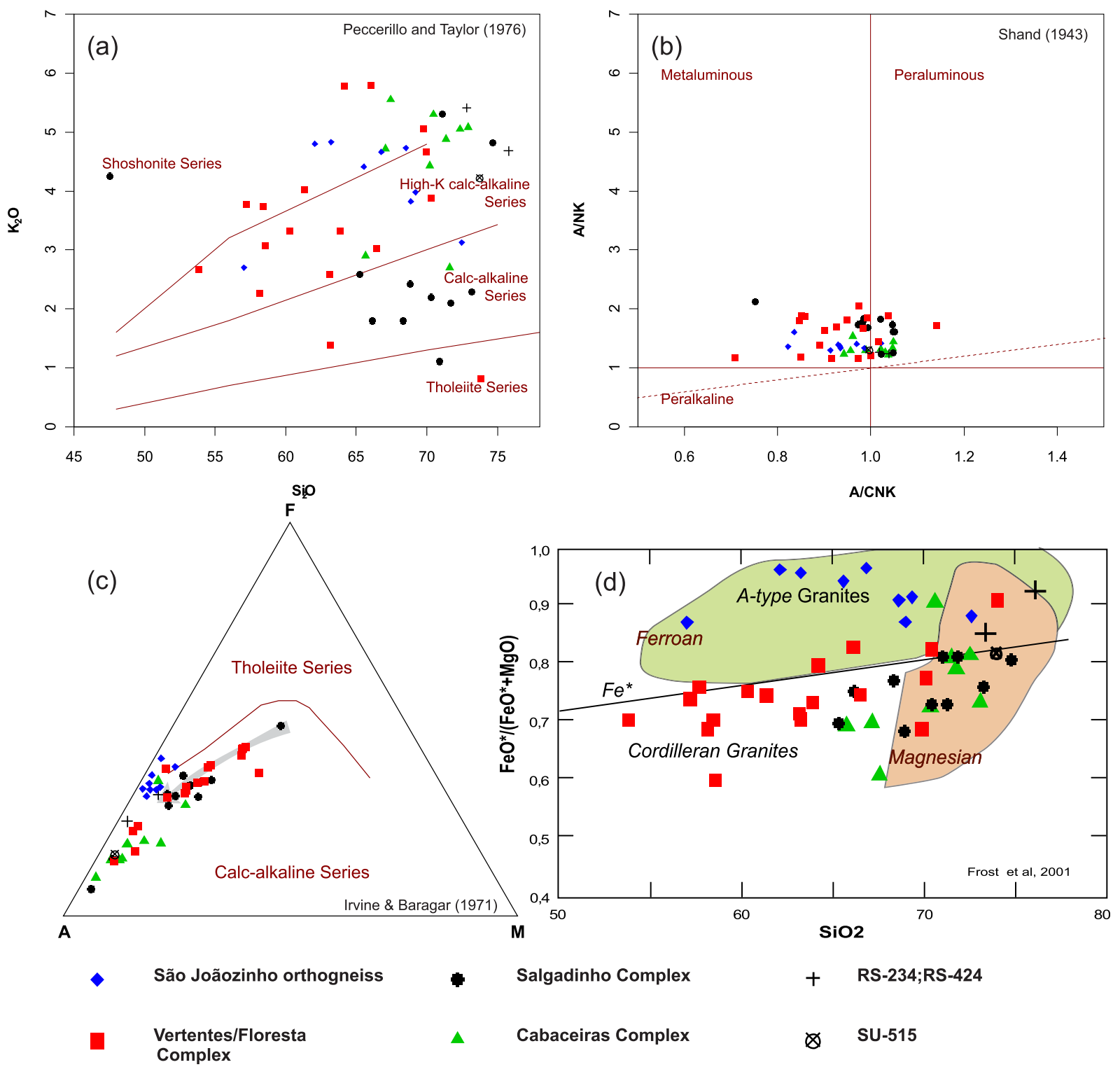

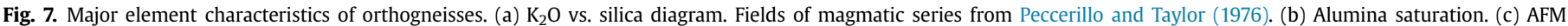

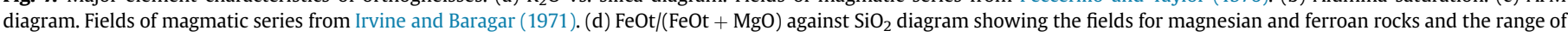
compositions of Cordilleran and A-type granites (Frost et al., 2001).

developed oscillatory zoning (Fig. 5c). The upper intercept of the discordia (1930 $\pm 49 \mathrm{Ma}$ ) obtained by regression of all data show large MSWD (11.8) but can be considered with caution to result from lead loss during a metamorphic and/or magmatic event around $1.93 \mathrm{Ga}$. This interpretation is supported by two analyses ((\#031-Z18 and \#031-Z22) with low discordance that gave ages of $1929 \pm 25 \mathrm{Ma}$ and $1925 \pm 22 \mathrm{Ma}$. In the first, the laser beam shot the core of a partially resorbed grain and, in the latter, a large overgrowth rim (Fig. 5c).

\subsection{Sm-Nd isotopic data}

Sm-Nd data are presented in Table 2, including those obtained in the present study and results from the literature, and a $\varepsilon_{\mathrm{Nd}}$ versus age diagram is shown on Fig. 6. An age of 2050 Ma was used for the calculation of $\varepsilon_{\mathrm{Nd}}(\mathrm{t})$ values. In the Cabaceiras Complex, the dated sample GL-476 yielded $\varepsilon_{\mathrm{Nd}}$ value of -7.92 and $\mathrm{Nd} \mathrm{T}_{\mathrm{DM}}$ model age of $2.74 \mathrm{Ga}$. Three other samples and previous results also yield negative
$\varepsilon_{\mathrm{Nd}}$ values, with Archean model ages ranging from 2.5 to $3.3 \mathrm{Ga}$. These results suggest participation of a Meso- to Neoarchean component in the genesis of this complex. Two analyses of the Salgadinho Complex gave similar Nd model ages of c. $2.6 \mathrm{Ga}$. Sample GL-479 from the São Joãozinho augen gneiss has $\mathrm{Nd} \mathrm{T}_{\mathrm{DM}}$ model age of $3.03 \mathrm{Ga}$ and $\varepsilon_{\mathrm{Nd}}$ value of -8.43 , which suggest derivation from reworked Mesoarchean crust. Banded gneisses of the Vertentes Complex have in general younger model ages than the granitic gneisses, mostly ranging from 2.2 to $2.5 \mathrm{Ga}$ and slightly negative to positive $\varepsilon_{\mathrm{Nd}}$ values, indicating derivation from more juvenile sources.

\section{Geochemistry}

Chemical analyses were performed at Acme Analytical Laboratories Ltd. in Canada. Major elements were determined using inductively coupled plasma-emission spectrometry with a detection limit of $0.01 \%$ and precision of $\pm 0.1 \%$. Trace and rare earth elements were analyzed using inductively coupled plasma-mass 

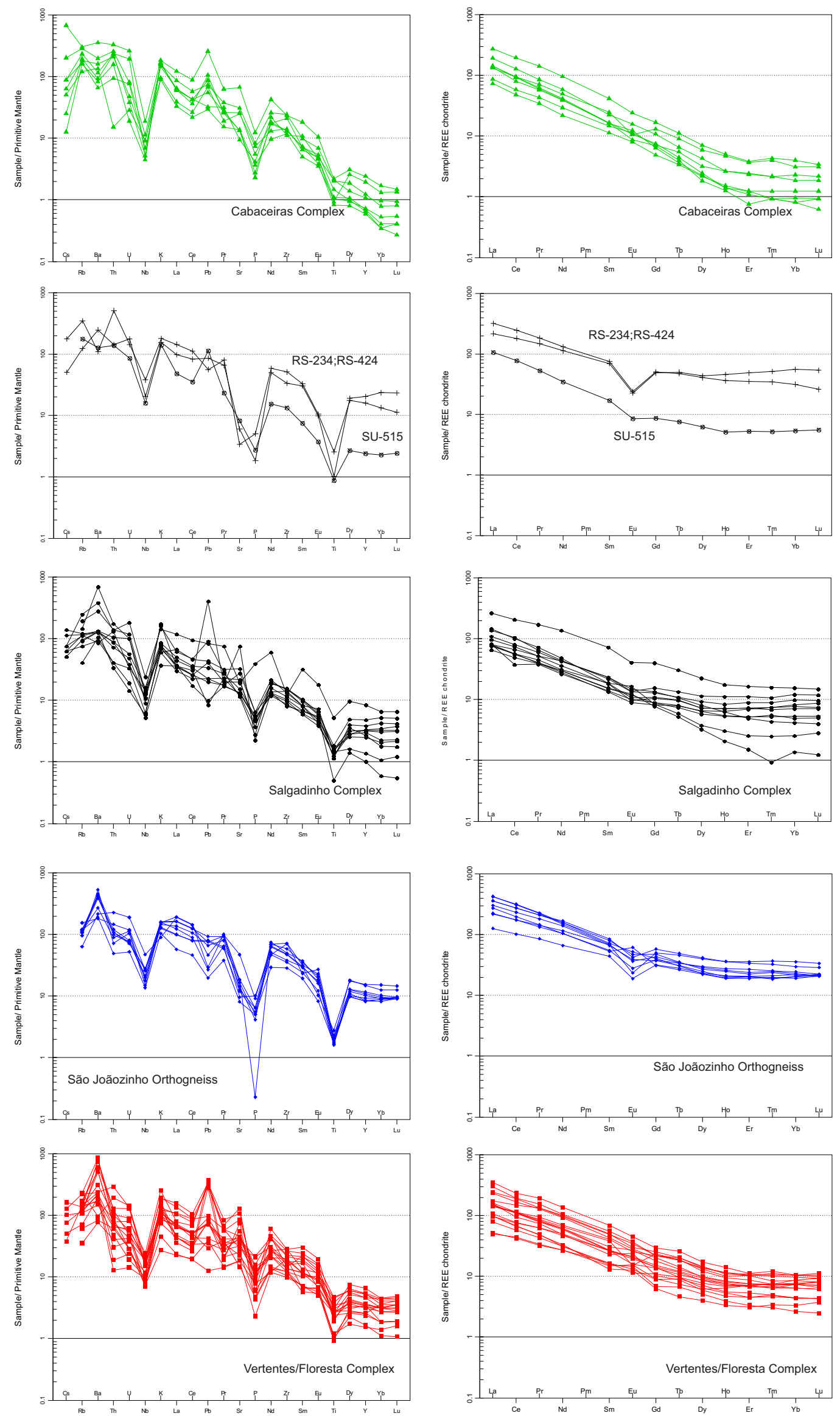

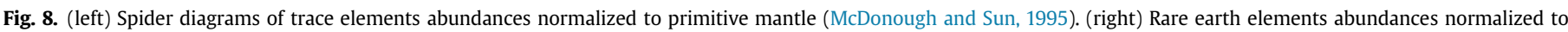
chondrite (Boynton, 1984). 


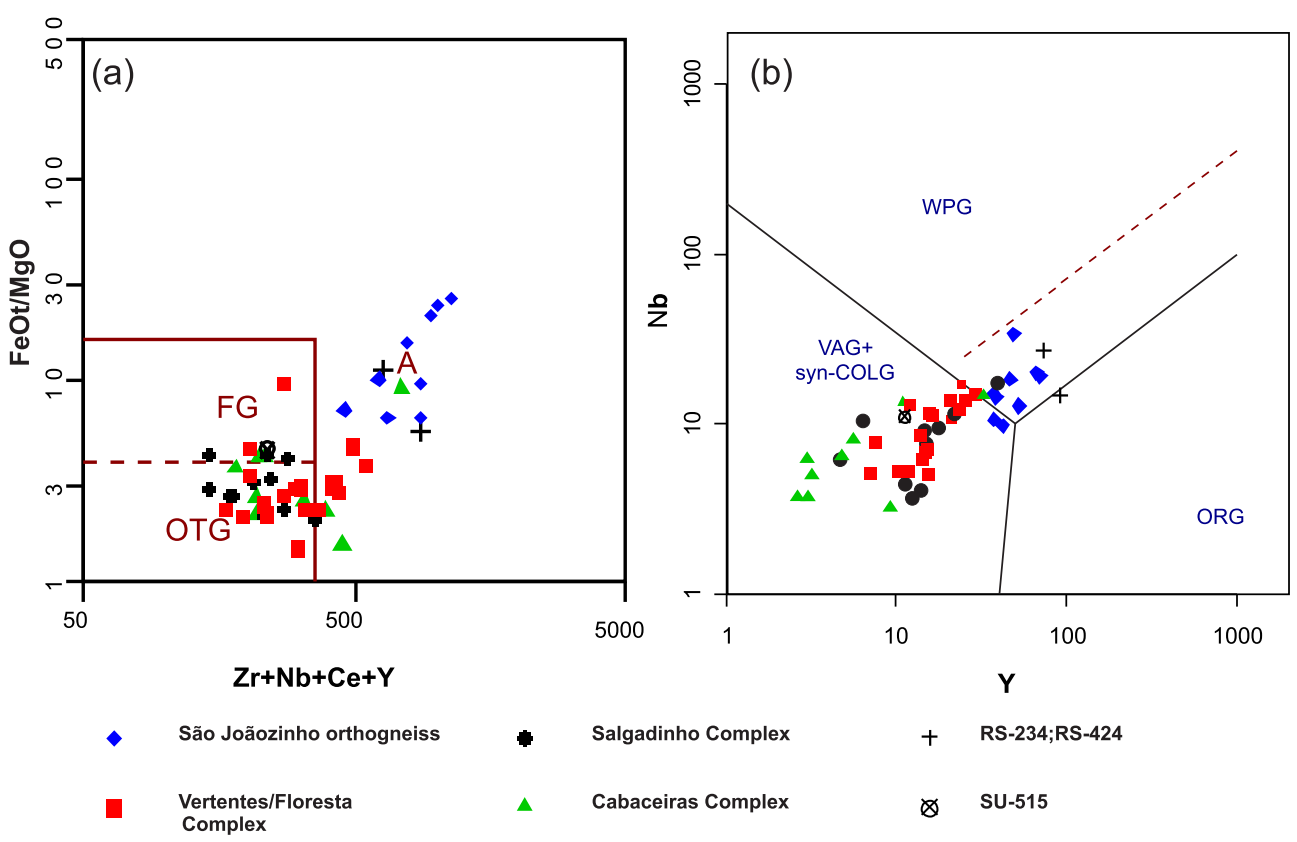

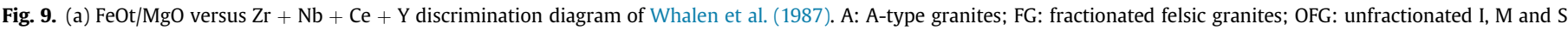

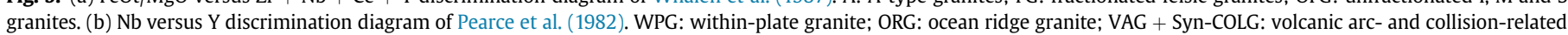
granites.

spectrometry (ICP-MS) with detection limits between 0.01 and $0.5 \mathrm{ppm}$ and precision of $\pm 5 \%$. Diagrams displaying the geochemical variability of the studied rocks were constructed using PetroGraph (Petrelli et al., 2005) and the latest version of open source software GCDkit 3.0 (Janoušek et al., 2006).

\subsection{Migmatitic orthogneisses}

The $\mathrm{SiO}_{2}$ contents of samples from the Cabaceiras and Salgadinho units range from 65 to 74 wt.\% (Table 3; Fig. 7a). Harker diagrams (not shown) exhibit low dispersion for most elements, indicating that, in spite of migmatization, significant mobility did not occur. The samples are metaluminous to slightly peraluminous (Fig. 7b) and plot in the field of magnesian granites (Fig. 7d). They follow the calc-alkaline trend in the AFM diagram (Fig. 7c), with samples of Cabaceiras unit extending closer to the A apex. This feature reflects the higher $\mathrm{K}_{2} \mathrm{O}$ content of the Cabaceiras samples as compared with samples from the Salgadinho Complex, as shown in the $\mathrm{K}_{2} \mathrm{O}$ versus $\mathrm{SiO}_{2}$ diagram (Fig. 7a): the first fall in the high- $\mathrm{K}$ calc-alkaline field and above this field whereas the latter belong to the medium-K calc-alkaline series.

As far as trace elements are concerned, the Cabaceiras and Salgadinho samples have typical features of calc-alkaline rocks, including high ratios of large ion lithophile elements (LILE) to high field strength elements (HFSE) and negative $\mathrm{Nb}, \mathrm{P}$ and Ti anomalies (Fig. 8). The main distinction between the two units is the higher contents of heavy rare earth elements (heavy REEs) and $\mathrm{Y}$ in the Salgadinho Complex (Fig. 8). The Salgadinho samples show moderate fractionation of the REEs, with a rapid decrease from La to Sm and a flattened pattern in the region of the heavy REE. In the Cabaceiras samples the REE patterns exhibit strong fractionation, with $(\mathrm{La} / \mathrm{Yb})_{\mathrm{N}}$ up to 200. In both cases, Eu negative anomalies are small or negligible. In tectonic discrimination diagrams, the samples plot in the field for volcanic arc granites (Fig. 9), according with the generally accepted setting for generation of calc-alkaline magmas (e.g., Brown, 1977; Barbarin, 1999; Frost et al., 2001).

In sparkling contrast with the Salgadinho and Cabaceiras complexes, the 2.18 Ga-old sample RS-234 and another sample of the same unit have high contents of heavy REEs, $\mathrm{Y}$ and $\mathrm{Nb}$ and low values of $\mathrm{Sr}$ and $\mathrm{Eu}$ (Table 3). These features make that the samples plot on the within-plate field in tectonic discrimination diagrams (Fig. 9). The samples exhibit chondrite normalized REE patterns characterized by well-marked negative Eu anomalies and a flat pattern from Gd to Lu (Fig. 8). These characteristics are typical of Atype granites (e.g., Whalen et al., 1987; Eby, 1992; Frost et al., 2001).

\subsection{São Joaozinho augen gneiss}

Samples of the São Joaozinho orthogneiss are metaluminous and ferroan (Fig. 7), and plot in the within plate field in tectonic discrimination diagrams (Fig. 9). The chondrite normalized REE patterns show $\mathrm{La}_{\mathrm{N}} / \mathrm{Yb}_{\mathrm{N}}$ ratio $<20$, with fractionated LREE and flat HREE patterns, and $\left(\mathrm{Eu} / \mathrm{Eu}^{*}\right)_{\mathrm{N}}$ between 0.40 and 0.97 (Fig. 8). The primitive mantle normalized multielemental diagrams show smaller LILE/HFSE fractionation than the Salgadinho and Cabaceiras units, with smaller negative anomaly of $\mathrm{Nb}$, and stronger depletion in $\mathrm{P}$ and $\mathrm{Ti}$ and positive peaks in $\mathrm{La}$ and $\mathrm{Zr}$. These features are similar to those of A-type granites.

\subsection{Banded gneisses (Vertentes Complex)}

Samples of the Vertentes Complex mainly show silica contents varying from 54 to 66 wt.\%. Four samples with $\mathrm{SiO}_{2} \geq 70$ wt.\% probably incorporated a large proportion of the felsic bands, which is difficult to avoid given the generally fine banding. The samples are metaluminous to slightly peraluminous, plot in the high-K calcalkaline field in the $\mathrm{K}_{2} \mathrm{O}$ versus $\mathrm{SiO}_{2}$ diagram, and follow the calcalkaline trend in the AFM diagram (Fig. 7). Chondrite normalized REE patterns are moderately to strongly fractionated (La/ $\mathrm{Yb})_{\mathrm{N}}=12-48$ ), showing a rapid decrease from La to Sm and a flat profile in the region of the heavy REEs (Fig. 8). Eu anomalies are generally small, with some samples showing negative anomalies and others positive anomalies. In primitive mantle normalized multielemental diagram (Fig. 8), the samples are characterized by negative anomalies of $\mathrm{Nb}, \mathrm{P}$ and $\mathrm{Ti}$. 
(a)

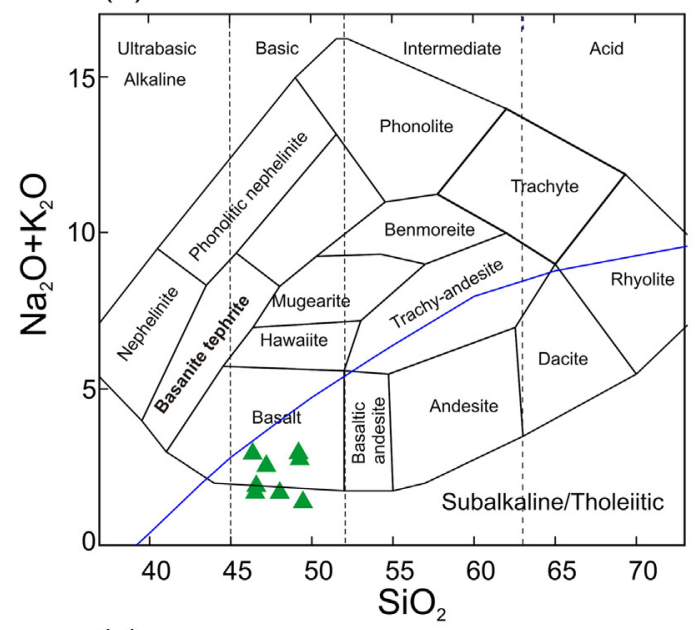

(c)

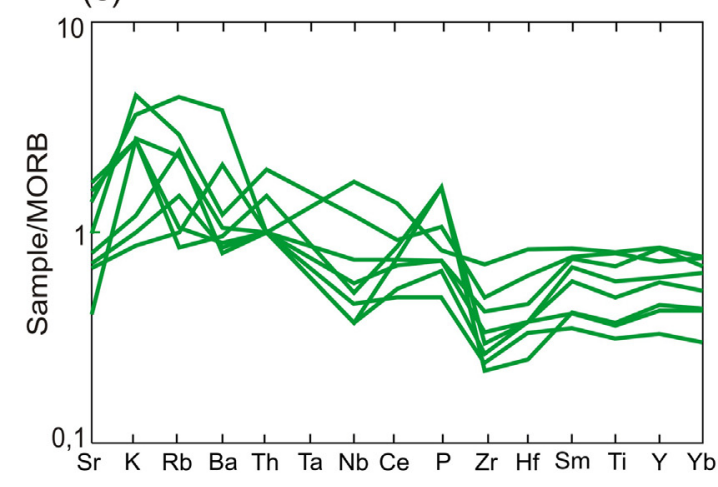

(e)

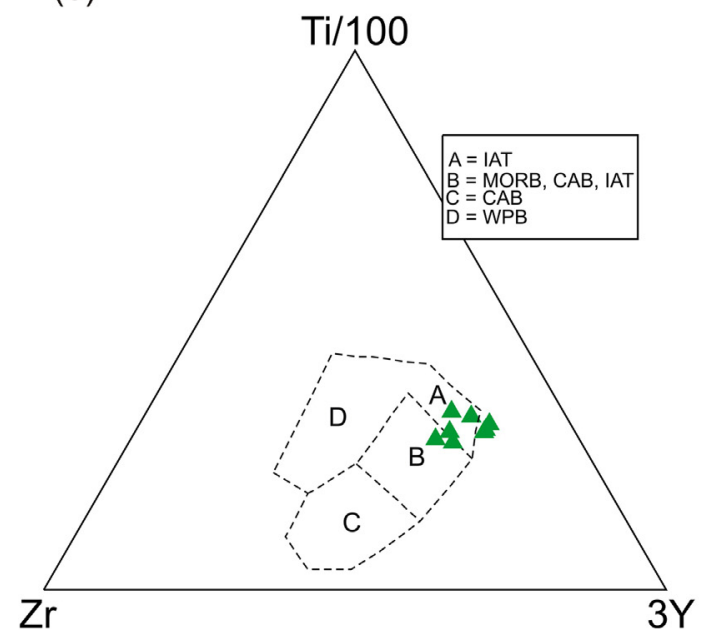

(b)

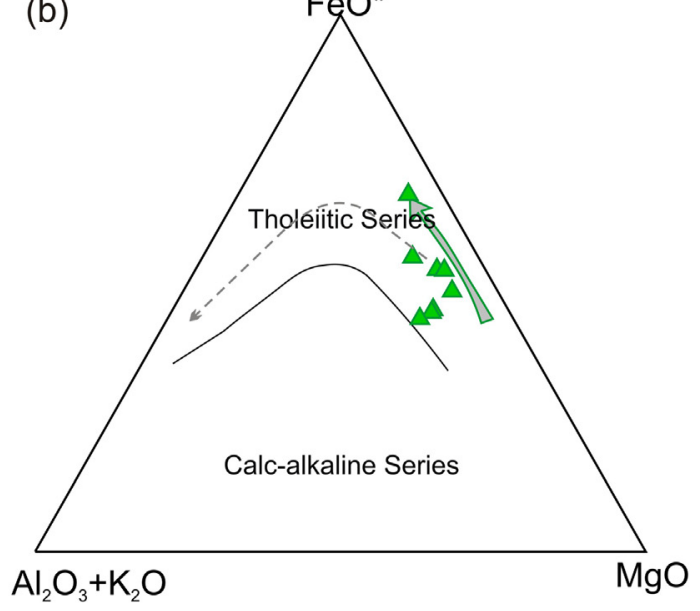

(d)

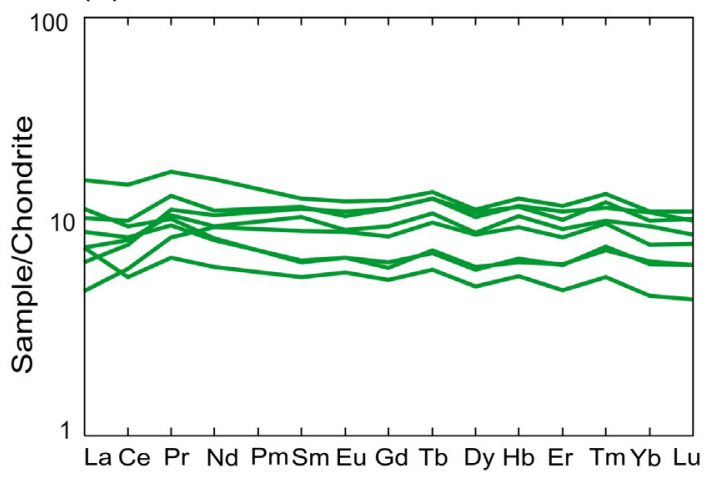

(f)

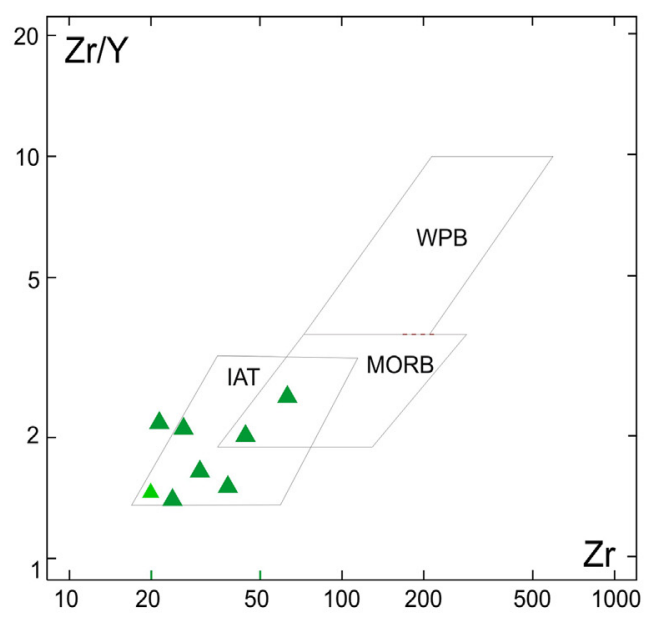

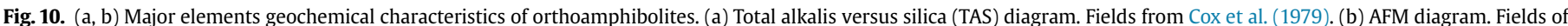

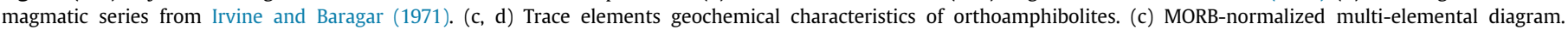

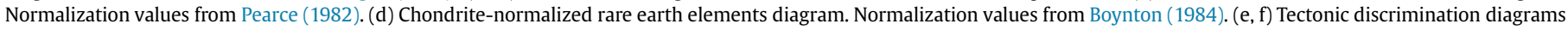
from (e) Pearce and Cann (1973) and (f) Pearce and Norry (1979). CAB: calc-alkalic basalt; IAT: island arc tholeiite; MORB: mid-ocean ridge basalt; WPB: within-plate basalt.

\subsection{Orthoamphibolites}

The metamafic rocks associated with the Cabaceiras unit display a narrow range of $\mathrm{SiO}_{2}\left(46-50\right.$ wt.\%) and $\mathrm{Na}_{2} \mathrm{O}+\mathrm{K}_{2} \mathrm{O}$ (1.5-3.5 wt.\%) contents (Fig. 10). They follow the trend of the tholeiitic series in the AFM diagram; show flat REE patterns, with REE contents about ten times chondrite values; and have high LILE and low HFSE as compared with MORB. In tectonic discrimination diagrams, they plot in the island arc field.

\subsection{Felsic gneiss}

A single sample of the felsic gneisss unit was analyzed (Table 3). It has high silica and total alkalis contents (73.7 and $7.7 \mathrm{wt} . \%$, 
respectively); exhibits a subtle negative Eu anomaly in the chondrite normalized REE diagram (Fig. 8 - sample SU-515), and projects in the field for fractionated granites in the $\mathrm{FeO}_{\mathrm{t}} / \mathrm{MgO}$ versus $\mathrm{Zr}+\mathrm{Nb}+\mathrm{Ce}+\mathrm{Y}$ diagram (Fig. 9a) and in the field of volcanic arc and syncollisional granites in the $\mathrm{Nb}$ versus $\mathrm{Yb}$ tectonic discrimination diagram (Fig. 9b).

\section{Discussion}

\subsection{Paleoproterozoic magmatic and metamorphic events}

Zircons from the dated samples record c. 200 million years of geological history, during which several magmatic and metamorphic events are recognized. The age of $2.18 \mathrm{Ga}$ of sample RS-234 is amongst the oldest found in the Central Domain, where ages

(a)
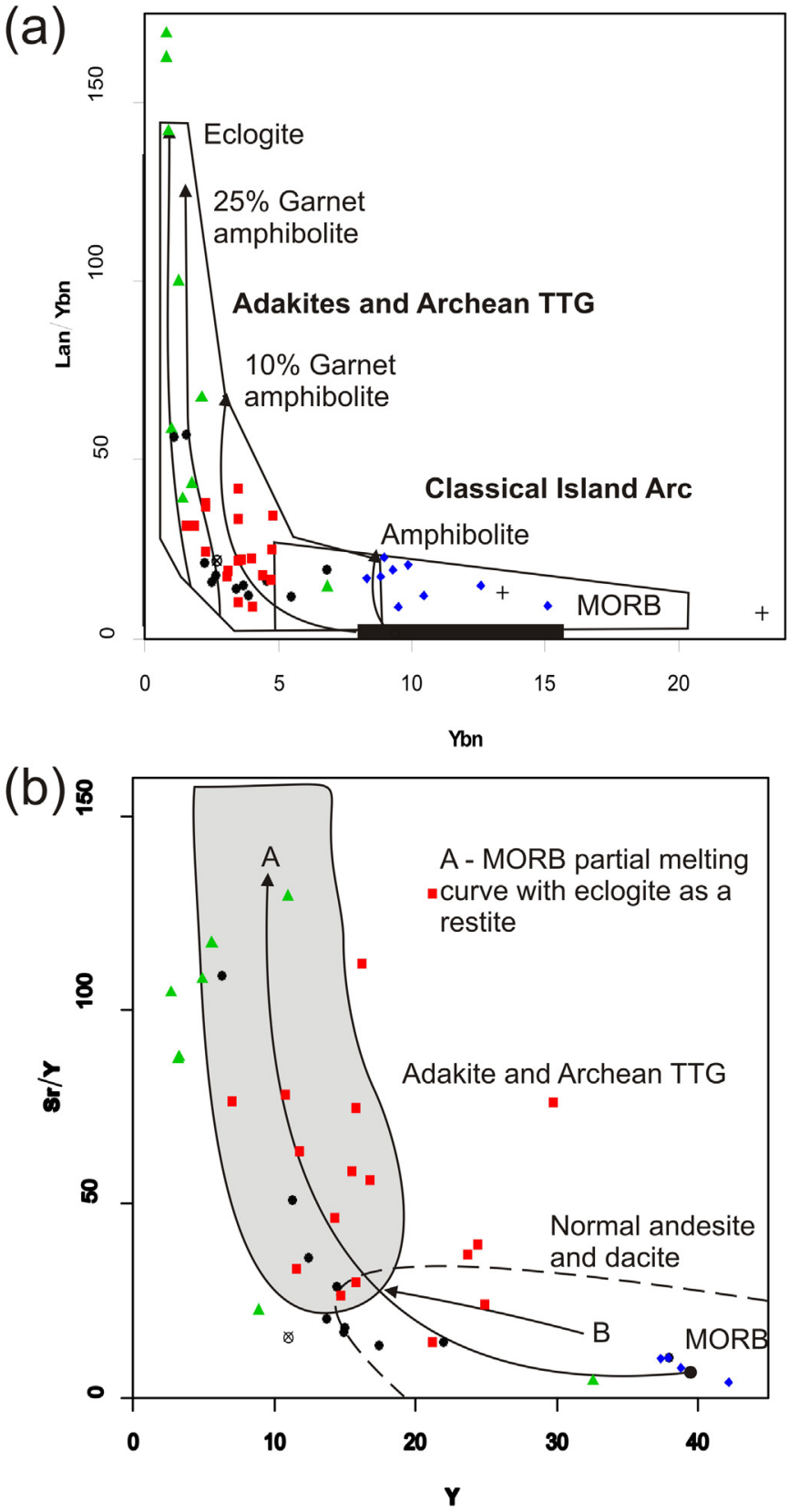

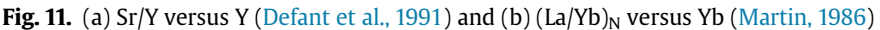
diagrams showing the fields for adakites and TTGs and for calc-alkaline rocks, and the curves for partial melting of mafic sources leaving garnet at the residue. younger than 2.15 Ga are more common (Van Schmus et al., 1995, 2011; Neves, 2015, and references cited in these works). This sample and nearby rocks are indistinguishable on field and petrographic basis from the much younger (c. $2.06 \mathrm{Ga}$ ) migmatitic orthogneisses of the Salgadinho and Cabaceiras complexes, which demonstrates the need for systematic geochronological and geochemical work in old gneissic terrains.

By large, the most voluminous magmatism in the study area is represented by the emplacement of the protoliths of the Vertentes, Salgadinho and Cabaceiras complexes. The age of the Vertentes complex obtained in this study $(2096 \pm 23 \mathrm{Ma})$ falls within the age range (2162-2093 Ma) reported in previous ones (Neves et al., 2006; Brito Neves et al., 2013). These data point out to an important magmatic event with duration of c. 70 Ma. Unexpectedly, the São Joaozinho orthogneiss has an age $(2109 \pm 15 \mathrm{Ma})$ that also falls within the range of ages available for the Vertentes Complex, despite their field, petrographic and geochemical dissimilarities. This finding implies that rocks with different geochemical characteristics are not necessarily emplaced in different times during the geological evolution of a given region.

The $>40 \mathrm{Ma}$ age difference between the Vertentes Complex and the Salgadinho/Cabaceiras complexes could suggest that magmatism was episodic rather than continuous. However, recent studies suggest that zircons formed in intraoceanic magmatic arcs are less prone to preservation than those generated during continental collisions (Hawkesworth et al., 2010; Dhuime et al., 2012; Cawood et al., 2013), such that this apparent absence of magmatism may be an artefact of the geological record. Another apparent magmatic lull is observed between $2042 \pm 11 \mathrm{Ma}$ and $1981 \pm 23 \mathrm{Ma}$, the ages of intrusion of the protoliths, respectively, of the orthoamphibolite and of the felsic gneiss. The latter age is similar within errors to that found (1991 $\pm 5 \mathrm{Ma}$ ) in one sample dated by Neves et al. (2006), attesting intrusion of magmas with clear crustal affinity c. 1.99-1.98 Ga ago.

A metamorphic event at c. $2.06-2.04 \mathrm{Ga}$ is indicated by the $2060 \pm 14$ Ma weighted average of five concordant ages and the $2044 \pm 27$ Ma upper intercept age of sample SU-541. A concordant analysis with an age of $2048 \pm 22 \mathrm{Ma}$ in a grain with low Th/U and the presence of faint oscillatory zoning and embayments cutting the concentric zoning in other grains (Fig. 5b) also support postcrystallization origin. The age of 2044 Ma of the upper intercept is identical to the one obtained by Neves et al. (2006) in a batch of analyses from another sample of the Vertentes complex whose zircon grains have similar characteristics, thus reinforcing the existence of this metamorphic event.

Sample SU-541 has three grains (two of which with low Th/U ratio) whose analyses have an upper intercept age of $1986 \pm 38 \mathrm{Ma}$. Similar result $(1983 \pm 20 \mathrm{Ma})$ was found by Brito Neves et al. (2013) on zircon overgrowths in one of the samples of the Vertentes Complex studied by them. Analyses of four grains with low $\mathrm{Th} / \mathrm{U}$ ratio from sample RS-423 yielded an upper intercept age of $1996 \pm 13$ Ma. These data indicate other metamorphic episode around $2.0-1.98 \mathrm{Ga}$. This time span coincides with the crystallization ages of felsic gneisses in the study area, showing that magmatism and metamorphism were broadly coeval. The geochemical characteristics of sample SU-515 suggest that these events are probably associated with collision. The occurrence or not of yet another metamorphic event around c. $1.93 \mathrm{Ga}$, as suggested by some analyses of sample SU-515, requires further investigation. Finally, imprints of Brasilano-age metamorphism is evidenced by the low intercept age of $604 \pm 20$ Ma from sample RS-234.

\subsection{Petrogenesis}

The dominantly intermediate composition, the metaluminous character, and the relatively high contents of compatible elements 
of the Vertentes Complex require that mafic or ultramafic sources are involved on its genesis. Partial melting of mafic protoliths appears unlikely because most dehydration-melting experiments of metabasalts have produced tonalitic to granitic melts rather than dioritic ones (e.g. Rushmer, 1991; Patiño-Douce e Beard, 1995). The high contents of LILE also exclude the depleted mantle as a possible source. Partial melting of an enriched mantle seems to be the better option, with the parental magmas evolving through fractional crystallization. The relatively juvenile Nd model ages suggests little or no interaction with preexisting continental crust.

Similarly to the Vertentes Complex, the Salgadinho and Cabaceiras units have relatively high contents of $\mathrm{MgO}, \mathrm{CaO}$ and $\mathrm{FeO}$, which exclude sedimentary or felsic igneous rocks as possible sources. On the other hand, ultramafic sources are implausible due to the essentially granitic composition. Derivation from mafic/intermediate sources by either partial melting or fractional crystallization is therefore the most likely hypothesis. In the Salgadinho Complex, the absence of both Eu anomalies and concave-up patterns in the region of the heavy REEs indicate limited participation of plagioclase and amphibole on its genesis. The REE patterns are similar to the samples of the Vertentes Complex, being richer in the heavy REE. This resemblance suggests partial melting of, or differentiation from, sources similar in compositions to the Vertentes Complex through retention/fractionation of mineral phases with low bulk distribution coefficients, such as olivine or pyroxene. In contrast, depletion of heavy REEs in the Cabaceiras unit strongly favors garnet and/or amphibole involvement (Fig. 11), suggesting genesis of the magmas at higher pressures than in the case of the Salgadinho Complex. As a consequence, despite the much higher $\mathrm{K}_{2} \mathrm{O}$ content of the Cabaceiras complex as compared with modern adakites and Archean tonalite-trondhjemite-granodiorite (TTG) suites, the high $\mathrm{Sr} / \mathrm{Y}$ and $(\mathrm{La} / \mathrm{Yb})_{\mathrm{N}}$ make the samples to plot in the field for these rocks in the Sr/Y versus Y (Defant et al., 1991) and (La/ $\mathrm{Yb})_{\mathrm{N}}$ versus $\mathrm{Yb}$ (Martin, 1986) diagrams (Fig.11).

The São Joaozinho orthogneiss has several geochemical affinities with A-type granites, particularly with the metaluminous Atype granites found in the Amazonian Craton (Dall'Agnoll and Oliveira, 2007). Petrogenetic modelling by Dall"Agnoll et al. (1999) suggested that magmas with these characteristics could have been produced by partial melting of clinopyroxene-bearing quartz diorites.

\subsection{Tectonic setting, crustal growth and reworking}

The geochemistry of the Vertentes, Salgadinho and Cabaceiras complexes is so similar to modern calc-alkaline magmas that generation of the parental magmas in a convergent setting seems to be an inescapable conclusion. The relatively young $\mathrm{Nd}$ model age of the Vertentes Complex favors intrusion in an intraoceanic arc rather than in an active continental margin. The presence of older inherited zircons (2.23-2.16 Ga-old) in sample SU-541 could mark the beginning of arc development. In contrast, the Salgadinho and Cabaceiras units, although also displaying subduction-related signature, have essentially granitic composition and slightly peraluminous character, which suggests emplacement in a more evolved setting, probably an active continental margin. The Archean Nd model ages of the Cabaceiras Complex suggest mixing between a juvenile component and an older, crustal, component. Existence of an ancient crustal component is also attested by the Nd model ages of the São Joaozinho orthogneiss and sample RS-234. The island-arc affinity of the orthoamphibolite indicates persistence of a convergent environment until at least $2042 \mathrm{Ma}$.

If the above inferences are correct, one important point to consider is why rocks with A-type geochemistry are present in a convergent setting. The recent discovery of rifted continental fragments in the Indian Ocean (Torsvik et al., 2013) and the presence of old zircons in juvenile intraoceanic arc terranes (Hargrove et al., 2006) suggest that occurrence of crustal material in oceanic realms may be more common than usually realized. Due to the older age of sample RS-234 $(2.18 \mathrm{Ga})$, it is possible that it belonged to such a fragment that was engulfed by a growing volcanic arc. The age of the São Joaozinho orthogneiss is similar to the age of the Vertentes Complex (c. 2.1 Ga) obtained in this study and poses a different problem. The apparent conundrum of occurrence in the same area of rocks of similar age and different chemical signatures may have several explanations. A-type granites are usually ascribed to intraplate ("anorogenic") settings, but occur in a variety of other settings, the post-collisional one being particularly common (e.g., Jung et al., 1998; Almeida et al., 2002; Bonin, 2007; Li et al., 2014). A-type granites are also described from convergent settings, such as the Lachlan Fold Belt of Australia (King et al., 1997), where their inferred high temperature of formation can be related to extension associated with slab breakoff during arc-arc collision (Yuan et al., 2010; Li et al., 2014), back-arc extension (King et al., 1997; Lister and Foster, 2009), subduction of ocean ridge segments, or delamination of the lower part of thick volcanic arcs. We therefore ascribe the genesis of the São Joãozinho orthogneiss to local extensional conditions in an otherwise convergent setting.

\subsection{Geodynamic hypothesis}

Our preferred scenario for the evolution of the study area starts with island arc construction around $2.2 \mathrm{Ga}$, leading to an expressive volcanic arc edifice by $2.13-2.10 \mathrm{Ga}$ (Fig. 12a-d). Strong negative initial $\varepsilon_{\mathrm{Nd}}$ and Archean $\mathrm{Nd}$ model ages for the São Joãozinho orthogneiss and the Cabaceiras Complex indicate that ancient continental material was involved in their genesis. Accordingly, we propose that a microcontinental fragment was assimilated by the growing magmatic arc (Fig. 12 b-d). Production of the parental magmas of the São Joaozinho orthogneiss and of sample RS-234 is attributed to melting of the old crustal component, possibly during periods of back-arc extension associated with slab retreat (Fig. 12c and f), thus explaining their affinity with A-type granites.

It is generally agreed that continents acquire a bulk andesitic composition by internal differentiation and foundering of mafic/ ultramafic cumulates (e.g., Kay and Kay, 1993; Jagoutz and Schmidt, 2013; Jagoutz and Behn, 2013). We propose that the Vertentes Complex resulted from the acting of these processes (Fig. 12d and e) and that, by $2.06 \mathrm{Ga}$, the crust had evolved enough to become intruded by magmas formed at the mantle wedge of the now largely continental magmatic arc (Fig. 12g). Orthogneisses from the Rio Grande do Norte Domain, which outcrop just to the north of the study area (Fig. 1c), have geochemical characteristics indicating intrusion in a continental arc setting (Souza et al., 2007) and ages older than 2.14 Ga (Hackspacher et al., 1990; Van Schmus et al., 1995; Dantas et la., 2004; Souza et al., 2007; Hollanda et al., 2011). We therefore speculate that accretion of the evolving oceanic arc to the active continental margin of the Rio Grande do Norte Domain led to cessation of subduction-related magmatic activity in this domain sometime between 2.15 and $2.13 \mathrm{Ga}$ (Fig. $12 \mathrm{~d}$ and e).

Although not a universal rule (Arculus, 1994), some arc magmas show a consistent relationship between alkalinity and depth to the Wadati-Benioff zone (e.g., Sakuyama and Nesbitt, 1986). Therefore, greater $\mathrm{K}_{2} \mathrm{O}$ and LILE contents and older $\mathrm{Nd}$ model ages in the Cabaceiras Complex as compared with the Salgadinho Complex (Figs. 6-8) are assumed to result from northward (present-day coordinates) subduction. Conterminous with intrusion of the protoliths of these complexes, high-temperature conditions were attained at mid crustal levels, being responsible for the 
(a)

(b)

$2.2-2.18 \mathrm{Ga}$
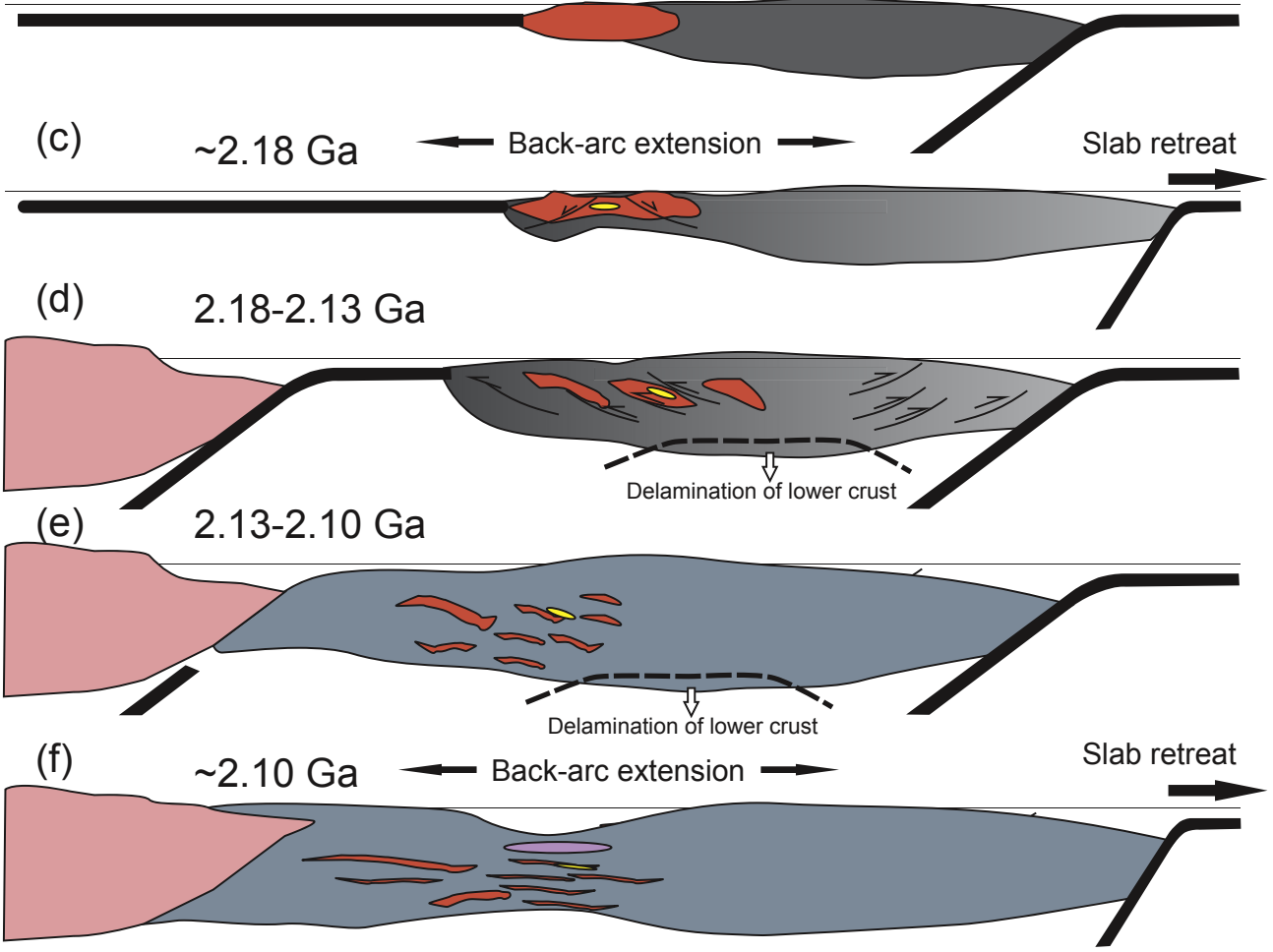

(g) $\quad 2.06-2.04 \mathrm{Ga}$

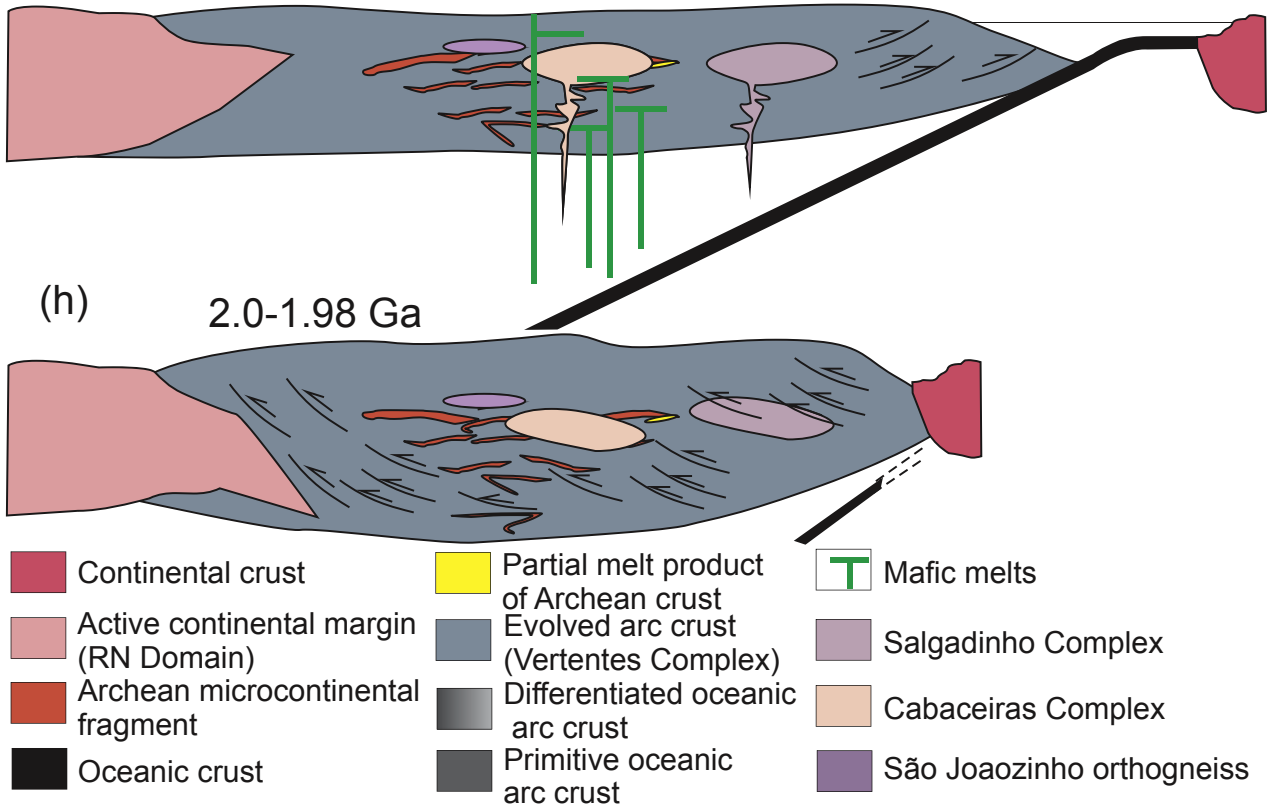

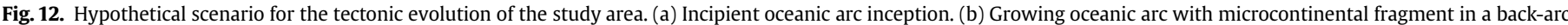

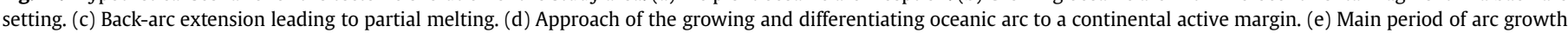

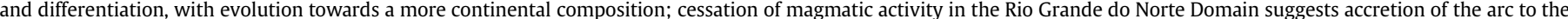

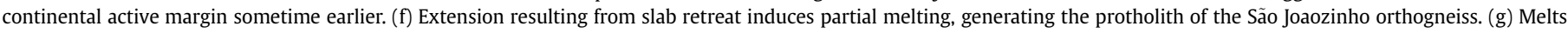

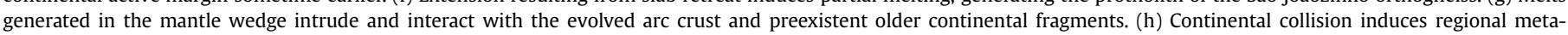
morphism and syncollisional magmatism. See text for discussion. 
metamorphic imprint observed on zircons of the Vertentes Complex (Fig. 5b).

The absence of rocks with ages between 2.04 and $2.01 \mathrm{Ga}$ in the study area could have been consequence of continental collision (Fig. 12g), which halted subduction-related magmatism. As a result of thickening-related heat production, by $2.0-1.98 \mathrm{Ga}$, regional temperatures had increased enough to allow crustal melting, syncollisional magmatism and metamorphism. These events were responsible for intrusion of the protolith of the felsic gneiss and for the metamorphic imprint observed in zircons of the Vertentes Complex and the orthoamphibolite.

\section{Conclusion}

The results of this study in conjunction with previous ones show that both crustal growth and recycling of older continental materials occurred in the eastern Borborema Province. A main period of crustal growth, represented by intrusion of calc-alkaline magmas of intermediate composition, is documented between 2.13 and $2.10 \mathrm{Ga}$. It was followed by intrusion of more evolved magmas containing variable contributions of older crustal sources at $c$. $2.06 \mathrm{Ga}$. Basalts (now orthoamphibolites) with island arc-related geochemistry indicate persistence of subduction zone magmatism until c. 2.04 Ga. Accordingly, a metamorphic event dated at c. 2.06-2.04 Ga is probably related with attainment of high temperature conditions at the deep levels of a thickened arc crust. In contrast, intrusion of felsic magmas coeval with regional metamorphism at 2.0-1.98 Ga probably records crustal thickening associated with continental collision.

\section{Acknowledgments}

SPN acknowledges support from the Brazilian agencies Conselho Nacional de Desenvolvimento Científico e Tecnológico (CNPq; grant 472582/2011-9) and Fundação de Amparo à Ciência e Tecnologia do Estado de Pernambuco (FACEPE; grant APQ-0479-1.07/06), and Dr. Cláudio Lamarão for providing access to the facilities of the Universidade Federal do Pará, where cathotoluminescence images of analyzed zircon crystals of samples SU-541, SU-49 and SU-515 were done. GAL and RGB acknowledge total support from Geological Survey of Brazil (CPRM), especially in fieldwork campaigns. The authors also thank constructive comments by two anonymous reviewers that helped to improve a previous version of the paper.

\section{References}

Dall gnoll, R., Oliveira, D.C., 2007. Oxidized, magnetite-series, rapakivi-type granites of Carajaa s, Brazil: Implications for classification and petrogenesis of A-type granites. Lithos 93, 215-233.

Almeida, F.F.M., Hasui, Y., Brito Neves, B.B., Fuck, R.A., 1981. Brazilian structural provinces: an introduction. Earth Sci. Rev. 17, 1-21.

Almeida, C.N., Guimarães, I.P., Da Silva Filho, A.F., 2002. A-type post-collisional granites in the Borborema province - NE Brazil: the Queimadas pluton. Gondwana Res. 5, 667-681.

Araújo, C.E.G., Cordani, U.G., Basei, M.A.S., Castro, N.A., Sato, K., Sproesser, W.M., 2012. U-Pb detrital zircon provenance of metasedimentary rocks from the Ceará Central and Médio Coreaú Domains, Borborema Province, NE-Brazil: tectonic implications for a long-lived Neoproterozoic active continental margin. Precambrian Res. 206-207, 36-51.

Archanjo, C.J., Hollanda, M.H.B.M., Rodrigues, S.W.O., Brito Neves, B.B. Armstrong, R., 2008. Fabrics of pre- and syntectonic granite plutons and chronology of shear zones in the Eastern Borborema Province, NE Brazil. J. Struct. Geol. 30, 310-326.

Arculus, R. 1994. Aspects of magma genesis in arcs. Lithos 33, 189-208.

Arthaud, M.H., Caby, R., Fuck, R.A., Dantas, E.L., Parente, C.V., 2008. Special Publications. Geology of the Northern Borborema Province, NE Brazil and its Correlation with Nigeria, NW Africa, vol. 294. Geological Society, London, pp. 49-67.

Barbarin, B., 1999. A review of the relationships between granitoid types, their origins and their geodynamic environments. Lithos 46, 605-626.
Bertrand, J.M.L., Lasserre, M., 1976. Pan-African and pre-Pan-African history of the Hoggar (Algerian Sahara) in the light of new geochronological data from the Aleksod area. Precambrian Res. 3, 343-362.

Bonin, B., 2007. A-type granites and related rocks: evolution of a concept, problems and prospects. Lithos 97, 1-29.

Boynton, W.V., 1984. Geochemistry of the rare earth elements: meteorite studies. In: Henderson, P. (Ed.), Rare Earth Element Geochemistry. Elsevier, Amsterdam, pp. 63-114.

Brasilino, R.G. Miranda, A.WA, Marinho, M.S, 2012. Programa Geologia do Brasil. Carta Geológica - Escala 1:100 000: Folha SB.24-Z-D-VI. CPRM - Servico Geológico do Brasil, Santa Cruz do Capibaribe.

Brito Neves, B.B., Campos Neto, M.C., Van Schmus, W.R., Santos, E.J., 2001a. O Sistema Pajeú-Paraíba e o Maciço São José do Campestre no leste da Borborema. Rev. Bras. Geociências 31, 173-184.

Brito Neves, B.B., Campos Neto, M.C., Van Schmus, W.R., Fernandes, T.M.G. Souza, S.L., 2001b. O terreno Alto Moxotó no leste da Paraíba (Maciço Caldas Brandão). Rev. Bras. Geociências 31, 185-194.

Brito Neves, B.B., Spröesser, W.M., Petronilho, L.A., Souza, S.L., 2013. Contribuição à Geologia e à Geocronologia do Terreno Rio Capibaribe (TRC, Província Borborema). In: Geologia USP, Série Científica, vol. 13, pp. 97-122.

Brown, G.C., 1977. Mantle origin of Cordilleran granites. Nature 265, 21-24.

Bruguier, O., Dada, S., Lancelot, J.R., 1994. Early Archean component (>3.5 Ga) within a 3.05 Ga orthogneiss from northern Nigeria: U-Pb zircon evidence. Earth Planet. Sci. Lett. 125, 89-103.

Buhn, B., Pimentel, M.M., Matteini, M., Dantas, E.L., 2009. High spatial resolution analysis of $\mathrm{Pb}$ and $\mathrm{U}$ isotopes for geochronology by laser ablation multi-collector inductively coupled plasma mass spectrometry (LA-MC-ICP-MS). An. Acad. Bras. Ciênc. 81, 1-16.

Cawood, P.A., Hawkesworth, C.J., Dhuime, B., et al., 2013. The continental record and the generation of continental crust. Geol. Soc. Am. Bull. 125, 14-32.

Cordani, U.G., Sato, K., 1999. Crustal evolution of the South American Platform, based on Nd isotopic systematics on granitoid rocks. Episodes 23 (3), 167-173.

Cox, K.G., Bell, J.D., Pankhurst, R.J., 1979. The Interpretation of Igneous Rocks. Allen \& Unwin, London.

Da Silva Filho, A.F., Guimarães, I.P., Van Schmus, W.R., 2002. Crustal evolution of the Pernambuco-Alagoas complex, Borborema Province, NE Brazil: Nd isotopic data from Neoproterozoic granitoids. Gondwana Res. 5, 409-422.

Dada, S., 2008. Special Publications. Proterozoic Evolution of the Nigeria-Borborema Province, vol. 294. Geological Society, London, pp. 121-136.

Dall'Agnol, R., Scaillet, B., Pichavant, M., 1999. An experimental study of a Lower Proterozoic A-type granite from the eastern Amazonian craton. J. Petrol. 40, $1673-1698$.

Dantas, E.L., 1997. Geocronologia U-Pb e Sm-Nd de Terrenos Arqueanos e Paleoproterozóicos do Maciço Caldas Brandão, NE do Brasil. Tese de Doutorado. UNESP, p. 208.

Dantas, E.L., Van Schmus, W.R., Hackspacher, P.C., Fetter, A., Brito Neves, B.B. Cordani, U.G., Nutman, A.P., Williams, I.S., 2004. The 3.4-3.5 Ga Sao José do Campestre massif, NE Brazil: remnants of the oldest crust in South America. Precambrian Res. 130, 113-137.

Defant, M.J., Richerson, P.M., de Boer, J.Z., Stewart, R.H., Maury, R.C., Bellon, H. Drummond, M.S., Feigenson, M.D., Jackson, T.E., 1991. Dacite genesis via both slab melting and differentiation: petrogenesis of La YeguadaVolcanic Complex, Panama. J. Petrol. 32, 1101-1142.

DePaolo, D.J., 1981. Trace element and isotopic effects of combined wall rock assimilation and fractional crystallization. Farth Planet Sci. Lett. 53, 189-202.

Dhuime, B., Hawkesworth, C.J., Cawood, P.A., Storey, C.D., 2012. A change in the geodynamics of continental growth 3 billion years ago. Science 335, 1334-1336.

Eby, G.N., 1992. Chemical subdivision of the A-type granitoids: petrogenetic and tectonic implications. Geology 20, 641-644.

Ferré, E., Déléris, J., Bouchez, J.-L., Lar, A.U., Peucat, J.-J., 1996. The Pan-African reactivation of Eburnean and Archean provinces in Nigeria: structural and isotopic data. J. Geol. Soc. Lond. 153, 719-728.

Ferreira, V.P., Sial, A.N., Jardim de Sá, E.F., 1998. Geochemical and isotopic signatures of Proterozoic granitoids in terranes of the Borborema structural province northeastern Brazil. J. South Am. Earth Sci. 11, 439-455.

Fetter, A.H., Van Schmus, W.R., Santos, T.J.S., Nogueira Neto, J.A., Arthaud, M.H., 2000. U-Pb and Sm-Nd geochronological constraints on the crustal evolution of basement architecture of Ceará state, NW Borborema province, NE Brazil: implications for the existence of the Paleoproterozoic supercontinent 'Atlantica'. Rev. Bras. Geociências 30, 102-106.

Frost, B.R., Barnes, C., Collins, W., Arculus, R., Ellis, D., Frost, C., 2001. A chemica classification for granitic rocks. J. Petrol. 42, 2033-2048.

Gióia, S.M.C.L., Pimentel, M.M., 2000. The Sm-Nd isotopic method in the geochronology laboratory of the University of Brasília. An. Acad. Bras. Ciências 72, 220-245.

Guimarães, I.P., Da Silva Filho, A.F., Almeida, C.N., Van Schmus, W.R., Araújo, J.M.M. Melo, S.C., Melo, E.B., 2004. Brasiliano (Pan-African) granite magmatism in the Pajeú-Paraíba belt, Northeast Brazil: an isotopic and geochronological approach. Precambrian Res. 135, 23-53.

Guimarães, I.P. Van Schmus, W.R., Brito Neves, Bittar, S.M.B., Da Silva Filho, A.F. Armstrong, R., 2012. U-Pb zircon ages of orthogneisses and supracrustal rocks of the Cariris Velhos belt: onset of Neoproterozoic rifting in the Borborema Province, NE Brazil. Precambrian Res. 192-195, 52-77.

Hackspacher, P.C., Van Schmus, W.R., Dantas, E.L., 1990. Um embasamento Transamazônico na Província Borborema. In: Nordeste, S.N. (Ed.), 36 Congr. Bras. Geol., Natal, pp. 2683-2696. 
Hargrove, U.S., Stern, R.J., Kimura, J.I., Manton, W.I., Johnson, P.R., 2006. How juvenile is the Arabian-Nubian Shield? Evidence from Nd isotopes and preNeoproterozoic inherited zircon in the Bi'r Umq suture zone, Saudi Arabia. Earth Planet. Sci. Lett. 252, 308-326.

Hawkesworth, C., Dhuime, B., Pietranik, A., Cawood, P., Kemp, T., Storey, C., 2010 The generation and evolution of the continental crust. J. Geol. Soc. Lond. 167, $229-248$.

Hollanda, M.H.B.M., Pimentel, M.M., Jardim de Sá, E.F., 2003. Paleoproterozoic subduction-related metasomatic signatures in the lithospheric mantle beneath $\mathrm{NE}$ Brazil: inferences from trace element and $\mathrm{Sr}-\mathrm{Nd}-\mathrm{Pb}$ isotopic compositions of Neoproterozoic high-K igneous rocks. J. South Am. Earth Sci. 15, 885-900.

Hollanda, M.H.B.M., Archanjo, C.J., Souza, L.C., Dunyi, L., Armstrong, R.A., 2011. Longlived Paleoproterozoic granitic magmatism in the Seridó-Jaguaribe domain, Borborema Province-NE Brazil. J. South Am. Earth Sci. 32, 287-300.

Irvine, T.N., Baragar, W.R., 1971. A guide to the chemical classification of the common volcanic rocks. Can. J. Earth Sci. 8, 523-548.

Jagoutz, O., Behn, M.D., 2013. Foundering of lower island-arc crust as an explanation for the origin of the continental Moho. Nature 504, 131-134.

Jagoutz, O., Schmidt, M.W., 2013. The composition of the foundered complement to the continental crust and are-evaluation of fluxes in arcs. Earth Planet. Sci. Lett. $371-372,177-190$

Janoušek, V., Farrow, C.M., Erban, V., 2006. Interpretation of whole-rock geochemical data in igneous geochemistry: introducing Geochemical Data Toolkit (GCDkit). J. Petrol. 47, 1255-1259.

Jung, S., Mezger, K., Hoernes, S., 1998. Petrology and geochemistry of syn- to postcollisional metaluminous A-type granites - a major and Nd-Sr-Pb-O-isotope study from the Proterozoic Damara Belt, Namibia. Lithos 45, 147-175.

Kay, R.W., Kay, S.M., 1993. Delamination and delamination magmatism. Tectonophysics 219, 177-189.

King, P.L., White, A.J.R., Chappell, B.W., Allen, C.M., 1997. Characterization and origin of aluminous A-type granites from the Lachlan Fold Belt, southeastern Australia. J. Petrol. 38, 371-391.

Kroner, A., 1977. The Precambrian geotectonic evolution of Africa: plate accretion versus plate destruction. Precambrian Res. 4, 163-213.

Lages, G.A., Marinho, M.S., 2012. Programa Geologia do Brasil. Carta Geológica Escala 1:100 000: Folha SB.24-Z-D-III, Boqueirão. CPRM - Serviço Geológico do Brasil.

Li, D., He, D., Shantosh, M., Tang, J., 2014. Petrogenesis of Late Paleozoic volcanics from the Zhaheba depression, East Junggar: Insights into collisional event in an accretionary orogeny of Central Asia. Lithos 184-187, 167-193.

Lister, G., Foster, M., 2009. Tectonic mode switches and the nature of orogenesis. Lithos 113, 274-291.

\section{Ludwig 2000.}

Mariano, G., Neves, S.P., Da Silva Filho, A.F., Guimarães, I.P., 2001. Diorites of the high-K calc-alkalic association: geochemistry and Sm-Nd data and implications for the evolution of the Borborema province, Northeast Brazil. Int. Geol. Rev. 43, 921-929.

Martin, H., 1986. Effect of steeper Archean geothermal gradient on geochemistry of subduction-zone magma. Geology 14, 753-756.

Martins, G., Oliveira, E.P., Lafon, J.M., 2009. The Algodões amphibolite-tonalite gneiss sequence, Borborema Province, NE Brazil: geochemical and geochronological evidence for Palaeoproterozoic accretion of oceanic plateau/back-arc basalts and adakitic plutons. Gondwana Res. 15, 71-85.

McDonough, W.F., Sun, S.S., 1995. The composition of the Earth. Chem. Geol. 120 $223-253$.

Medeiros, V.C., Medeiros, W.E., Jardim de Sá, E.F., 2011. Utilização de imagens aerogamaespectrométricas, Landsat $7 \mathrm{ETM}+$ e aeromagnéticas no estudo do arcabouc.o crustal da porção central do Domínio da Zona Transversal, Província Borborema, NE do Brasil. Rev. Bras. Geofísica 29, 83-97.

Neves, S.P., 2015. Constrains from zircon geochronology on the tectonic evolution of the Borborema Province (NE Brazil): widespread Neoproterozoic intracontinental reworking of a Paleoproterozoic accretionary orogen. J. South Am. Earth Sci. 58, 150-164.

Neves, S.P., Alcantara, V.C., 2010. Geochemistry of orthogneisses and metasedimentary rocks across a proposed terrane boundary in the Central Domain of Borborema Province, NE Brazil: geodynamic implications. J. South Am. Earth Sci. 29, 498-511.

Neves, S.P., Mariano, G., 1999. Assessing the tectonic significance of a large-scale transcurrent shear zone system: the Pernambuco lineament, northeastern Brazil. J. Struct. Geol. 21, 1369-1383.

Neves, S.P., Vauchez, A., 1995. Successive mixing and mingling of magmas in a plutonic complex of Northeast Brazil. Lithos 34, 275-299.

Neves, S.P., Vauchez, A., Feraud, G., 2000. Tectono-thermal evolution, magma emplacement, and shear zone development in the Caruaru area (Borborema Province, NE Brazil). Precambrian Res. 99, 1-32.

Neves, S.P., Melo, S.C., Moura, C.A.V., Mariano, G., Silva, J.M.R., 2004. Zircon Pb-Pb geochronology of the Caruaru area, northeastern Brazil: temporal constraints on the Proterozoic evolution of Borborema Province. Int. Geol. Rev. 46, 52-63.

Neves, S.P., Silva, J.M.R., Mariano, G., 2005. Oblique lineations in orthogneisses and supracrustal rocks: vertical partitioning of strain in a hot crust (eastern Borborema Province, NE Brazil). J. Struct. Geol. 27, 1513-1527.

Neves, S.P., Bruguier, O., Vauchez, A., Bosch, D., Silva, J.M.R., Mariano, G., 2006 Timing of crust formation, deposition of supracrustal sequences, and Transamazonian and Brasiliano metamorphism in the East Pernambuco belt (Borborema Province, NE Brazil): Implications for western Gondwana assembly. Precambrian Res. 149, 197-216.
Neves, S.P., Bruguier, O., Bosch, D., Silva, J.M.R., Mariano, G., 2008. U-Pb ages of plutonic and metaplutonic rocks in southern Borborema Province (NE Brazil): timing of Brasiliano deformation and magmatism. J. South Am. Earth Sci. 25, 285-297.

Neves, S.P., Bruguier, O., Silva, J.M.R., Bosch, D., Alcantara, V.C., Lima, C.M., 2009. The age distributions of detrital zircons in metasedimentary sequences in eastern Borborema Province (NE Brazil): evidence for intracontinental sedimentation and orogenesis? Precambrian Res. 175, 187-205.

Neves, S.P., Mariano, G. Silva, J.M.R., 2010. Programa Geologia do Brasil. Carta Geológica - Escala 1:100 000: Folha SB.25-Y-C-IV, Surubim. CPRM - Serviço Geológico do Brasil.

Njiosseu, E.L.T., Nzenti, J.P., Njanko, T., Kapajika, B., Nédélec, A., 2005. New U-Pb zircon ages from Tonga (Cameroon): coexisting Eburnean-Transamazonian (2.1 Ga) and Pan-African (0.6 Ga) imprints. Comptes Rendus Geosci. 337, 551-562.

Patiño Douce, A.E., Beard, J.S., 1995. Dehydration-melting of biotite gneiss and quartz amphibolite from 3 to 15 kbar. J. Petrol. 36 (3), 707-738.

Pearce, J.A., 1982. Trace element characteristics of lavas from destructive plate boundaries. In: Thorpe, R.S. (Ed.), Andesites: Orogenic Andesites and Related Rocks. Wiley, Chichester, pp. 525-548.

Pearce, J.A., Cann, J.R., 1973. Tectonic setting of basic volcanic rocks determined using trace element analyses. Earth Planet. Sci. Lett. 19, 290-300.

Pearce, J.A., Norry, M.J., 1979. Petrogenetic implications of Ti, Zr, Y, and Nb variations in volcanic rocks. Contrib. Mineral. Petrol. 69, 33-47.

Peccerillo, R., Taylor, S.R., 1976. Geochemistry of Eocene cal-alkaline volcanic rocks from the Kastamonu area, northern Turkey. Contrib. Mineral. Petrol. 58, 63-81.

Petrelli, M., Poli, G., Perugini, D., Peccerillo, A., 2005. PetroGraph: a new software to visualize, model, and present geochemical data in igneous petrology. Geochem. Geophys. Geosyst. 6, Q07011. http://dx.doi.org/10.1029/2005GC000932.

Rodrigues, S.W.O., Brito Neves, B.B., 2008. Padrões isotópicos Sm-Nd no limite entre os terrenos Alto Pajeú e Alto Moxotó (PB). Rev. Bras. Geociências 38, 209-225.

Rodrigues, S.W.O., Medeiros, V.C., Brasilino, R.G., 2010a. U/Pb Geochonology of Salvador Orthogneiss (Paleoproterozoic unit of Transversal zone, Borborema Province) in Campina Grande sheet (SB.25-Y-C-I). In: VII South American Symposium on Isotope Geology. CD-ROM, Brasília.

Rodrigues, S.W.O., Medeiros, V.C., Brito Neves, B.B., Marinho, M.S., Gusmão, R.O., 2010b. Programa Geologia do Brasil. Carta Geológica - Escala 1:100 000: Folha SB.25-Y-C-I, Campina Grande. CPRM - Servico Geológico do Brasil.

Rushmer, T., 1991. Partial melting of two amphibolites: contrasting experimental results under fluid-absent conditions. Contrib. Mineral. Petrol. 107, 41-59.

Sá, J.M., Bertrand, J.M., Leterrier, J., Macedo, M.H.F., 2002. Geochemistry and geochronology of pre-Brasiliano rocks from the Transversal Zone, Borborema Province, Northeast Brazil. J. South Am. Earth Sci. 14, 851-866.

Sakuyama, M., Nesbitt, R.W., 1986. Geochemistry of the Quaternary volcanic rocks of the northeast Japan arc. J. Volcanol. Geotherm. Res. 29, 413-450.

Santos, E.J., Nutman, A.P., Brito Neves, B.B., 2004. Idades SHRIMP U-Pb do Complexo Sertânia: implicações sobre a evolução tectônica da zona transversal, Província Borborema. In: Geologia USP: Série Científica, vol. 4, pp. 1-12.

Santos, T.J.S., Fetter, A.H., Hackspacher, P.C., Van Schmus, W.R., Nogueira Neto, J.A. 2008. Neoproterozoic tectonic and magmatic episodes in the NW sector of Borborema Province, NE Brazil, during assembly of Western Gondwana. J. South Am. Earth Sci. 25, 271-284.

Santos, E.J., Souza Neto, J.A., Carmona, L.C.M., Armstrong, R., Santos, L.C.M.L., Mendes, L.U.S., 2013. The metacarbonate rocks of Itatuba (Paraíba): a record of sedimentary recycling in a Paleoproterozoic collision zone of the Borborema province, NE Brazil. Precambrian Res. 224, 454-471.

Souza, Z.S., Martin, H., Peucat, J.J., Jardim de Sá, E.F., Macedo, M.H.F., 2007. Calcalkaline magmatism at the Archean-Proterozoic Transition: the Caicó Complex basement (NE Brazil). J. Petrol. 48, 2149-2185.

Torsvik et al, 2013. A Precambrian microcontinent in the Indian Ocean. Nat. Geosci. 6, 223-227.

Toteu, S.F., Van Schmus, W.R., Penaye, J., Michard, A., 2001. New U-Pb and Sm-Nd data from north-central Cameroon and its bearing on the pre-Pan African history of central Africa. Precambrian Res. 108, 45-73.

Van Schmus, W.R., Brito Neves, B.B., Hackspacher, P., Babinski, M., 1995. U/Pb and $\mathrm{Sm} / \mathrm{Nd}$ geochronologic studies of the eastern Borborema Province, northeastern Brazil: initial conclusions. J. South Am. Earth Sci. 8, 267-288.

Van Schmus, W.R., Brito Neves, B.B., Williams, I.S., Hackspacher, P., Fetter, A.H., Dantas, E.L., Babinski, M., 2003. The Seridó Group of NE Brazil, a late Neoproterozoic pre- to syn-collisional basin in West Gondwana: insights from SHRIMP U-Pb detrital zircon ages and Sm-Nd crustal residence (TDM) ages. Precambrian Res. 127, 287-327.

Van Schmus, W.R., Oliveira, E.P., Da Silva Filho, A., Toteu, S.F., Penaye, J., Guimarães, I.P., 2008. Special Publications. Proterozoic Links between the Borborema Province, NE Brazil, and the Central African Fold Belt, vol. 294. Geological Society, London, pp. 69-99.

Van Schmus, W.R., Kozuch, M., Brito Neves, B.B., 2011. Precambrian history of the Zona Transversal of the Borborema Province, NE Brazil: Insights from Sm-Nd and U-Pb geochronology. J. South Am. Earth Sci. 31, 227-252.

Whalen, J.B., Currie, K.L., Chappell, B.W., 1987. A-type granites: geochemical characteristics, discrimination and petrogenesis. Contrib. Mineral. Petrol. 95, 407-419.

Yuan, C., Sun, M., Wilde, S., X iao, W., Xu, Y., Long, X., Zhao, G., 2010. Post-collisional plutons in the Balikun area, East Chinese Tianshan: evolving magmatism in response to extension and slab break-off. Lithos 119, 269-288. 